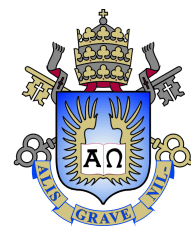

Henrique Reis Santiago

Development of a plugin based simulator for wellbore stability analysis and mud weight window optimization

Dissertação de Mestrado

Dissertation presented to the Programa de Pós-graduação em Engenharia Mecânica, do Departamento de Engenharia Mecânica da PUC-Rio in partial fulfillment of the requirements for the degree of Mestre em Engenharia Mecânica.

Advisor : Prof. Ivan Fabio Mota de Menezes Co-advisor: Dsc. André Luis Müller 

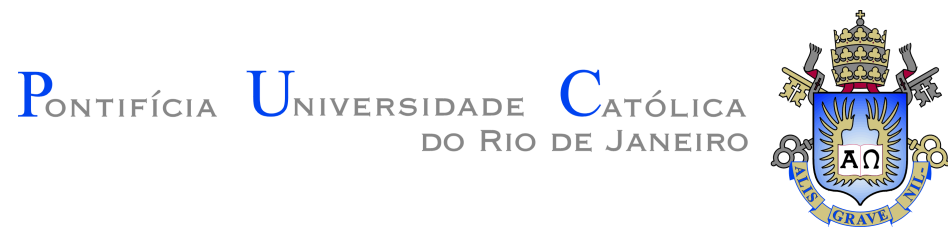

Henrique Reis Santiago

\section{Development of a plugin based simulator for wellbore stability analysis and mud weight window optimization}

Dissertation presented to the Programa de Pós-graduação em Engenharia Mecânica da PUC-Rio in partial fulfillment of the requirements for the degree of Mestre em Engenharia Mecânica. Approved by the Examination Committee:

Prof. Ivan Fabio Mota de Menezes

Advisor

Departamento de Engenharia Mecânica - PUC-Rio

Dsc. André Luis Müller

Co-advisor

Instituto Tecgraf de Desenvolvimento de Software Técnico-Cientifico da PUC-Rio - Tecgraf/PUC-Rio

Dsc. Luis Carlos de Sousa Junior

Petróleo Brasileiro S.A. - Petrobras

Dsc. Marcos Roberto Fetter Lopes

Grupo de Inversão e Imageamento Sísmico, Universidade

Federal Fluminense - GISIS/UFF

Rio de Janeiro, April the 30th, 2021 
All rights reserved.

\section{Henrique Reis Santiago}

Graduou-se em Engenharia Mecânica pela PUC-Rio. Trabalha no instituto Tecgraf no grupo de Modelagem Geomecânica. As áreas de interesse envolvem mecânica computacional, método dos elementos finitos, métodos numéricos e desenvolvimento de software.

Bibliographic data

Santiago, Henrique Reis

Development of a plugin based simulator for wellbore stability analysis and mud weight window optimization / Henrique Reis Santiago; advisor: Ivan Fabio Mota de Menezes; co-advisor: André Luis Müller. - 2021.

102 f: il. color. ; $30 \mathrm{~cm}$

Dissertação (mestrado) - Pontifícia Universidade Católica do Rio de Janeiro, Departamento de Engenharia Mecânica, 2021.

Inclui bibliografia

1. Engenharia Mecânica - Teses. 2. Método dos Elementos finitos. 3. Acoplamento Poromecânico. 4. Framework de Plugin. 5. Análise de estabilidade de poço. I. Menezes, Ivan Fabio. II. Müller, André Luis. III. Pontifícia Universidade Católica do Rio de Janeiro. Departamento de Engenharia Mecânica. IV. Título. 


\section{Acknowledgments}

To all my family for their continued support, specially throughout these harsh lockdown times. To my father, Carlos Alberto Santiago, and my brother, Guilherme Santiago, for being by my side everyday and allowing me the oportunity to study and be a better person.

To my advisor Ivan Menezes and co-advisor André Luis Müller for the constant challenges during the development of this work and for all the knowleadge passed on by both of you in the last two years.

To Marcio Santi and all my colleagues at the Geological Modeling Group. To be a part of this group since the start of my internship until now has been truly an unique oportunity. During these almost four years, you all helped me to become a better developer, engineer and professional.

To Leonardo Duarte for all the assistance during the early development stages of the plugin simulator, introducing me to the basic concepts of the TopSim framework and the TopS library.

To all my friends from college and high school. A special thanks to Baiano, Milhouse, Cunha, Sagrilo, Marcelo, Bastos and Lelê and to my lifelong friends Frazão, Pagliusi, Tarik, Neves, João and Bernardo for listening to me and being there for me during all the hard moments. Thank you so much for believing in me and for making my life happier.

This study was financed in part by the Coordenação de Aperfeiçoamento de Pessoal de Nível Superior - Brasil (CAPES) - Finance Code 001 


\section{Abstract}

Santiago, Henrique Reis; Menezes, Ivan Fabio (Advisor); Müller, André Luis (Co-Advisor). Development of a plugin based simulator for wellbore stability analysis and mud weight window optimization. Rio de Janeiro, 2021. 102p. Dissertação de Mestrado - Departamento de Engenharia Mecânica, Pontifícia Universidade Católica do Rio de Janeiro.

Well planning presents a major challenge in the oil \& gas industry. Wellbore stability analysis is one of the most important steps during well planning and provides the technical basis for a safe drilling operation. Therefore, comprehending and predicting the physical response of wells is extremely valuable to drilling engineers. In order to achieve wellbore stability, drilling operators control, among other factors, the internal wellbore pressure using the perforation mud weight. The mud pressure is used to balance the stresses at the wellbore region and its value must remain inside a stable window to ensure a safe operation. In this work, we propose a novel simulator to perform wellbore stability analysis and to compute the optimal mud pressure window. The simulator utilizes a plugin architecture, which provides a more flexible environment to develop and extend the simulator or even perform different analyses by exchanging one or more plugins. The ideal internal pressure and mud weight window are computed by solving a root-finding problem based on different failure criteria for the well. The simulator uses the Finite Element Method to solve each geomechanical analysis during the solution procedure, assuming the fluid-mechanical coupling and elastoplastic behavior around the wellbore. The simulator was validated using various examples and wellbore stability analyses were performed on a set of case studies from the literature.

\section{Keywords}

Finite Element Method; Poromechanical Coupling; Plugin Framework; Wellbore Stability Analysis. 


\section{Resumo}

Santiago, Henrique Reis; Menezes, Ivan Fabio; Müller, André Luis. Desenvolvimento de um simulador baseado em plugin para otimização da janela de estabilidade de poços de petróleo. Rio de Janeiro, 2021. 102p. Dissertação de Mestrado - Departamento de Engenharia Mecânica, Pontifícia Universidade Católica do Rio de Janeiro.

O projeto de poços de petróleo representa um dos maiores desafios na indústria de óleo e gás. A análise de estabilidade é parte fundamental do projeto pois fornece subsídios para perfuração e operação dos poços. Assim, compreender e prever a resposta geomecânica de um poço durante sua perfuração e produção é de suma importância. Para que o poço permaneça estável durante a perfuração, busca-se controlar, dentre outros fatores, a pressão interna ao longo de todo o processo com a lama de perfuração. Ela tem como objetivo manter a região do furo em equilíbrio, próximo de seu estado pré perfuração. A pressão interna no poço também deve permanecer dentro de uma janela de estabilidade, de forma que a perfuração ocorra de forma segura. Neste trabalho, é proposto um novo simulador para a obtenção da janela de estabilidade ótima para perfuração de poços de petróleo. O simulador utiliza uma arquitetura de plugins, que o permite realizar diferentes análises apenas substituindo alguns plugins e garante maior flexibilidade para desenvolver e estender o simulador se comparado com outros disponíveis num formato de caixa preta. O cálculo da pressão ótima da janela de estabilidade é realizado por meio da obtenção de raízes de funções de restrição baseadas em critérios de falha por plastificação ou fratura do poço. Para resolver as análises geomecânicas envolvidas, o simulador utiliza o Método dos Elementos Finitos considerando o acoplamento fluido mecânico e o comportamento elastoplástico no entorno do poço. São apresentados exemplos para a validação do simulador, bem como análises de estabilidade de poços baseados em estudos de caso da literatura.

\section{Palavras-chave}

Método dos Elementos finitos; Acoplamento Poromecânico; Framework de Plugin; Análise de estabilidade de poço. 


\section{Table of contents}

1 Introduction $\quad 13$

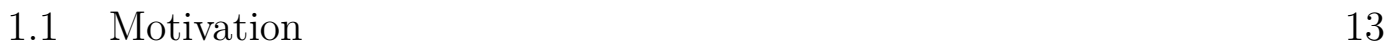

$\begin{array}{lll}1.2 & \text { Common Issues } & 14\end{array}$

$\begin{array}{lll}1.3 & \text { Objectives } & 16\end{array}$

$\begin{array}{lll}1.4 \text { Outline } & 16\end{array}$

2 Theory $\quad 18$

2.1 Mechanical Equilibrium 18

$\begin{array}{lll}2.1 .1 & \text { Yield Criteria } & 20\end{array}$

2.1.1.1 von Mises 21

2.1.1.2 Mohr-Coulomb 22

2.1.1.3 Modified Cam Clay 24

$\begin{array}{lll}2.2 & \text { Fluid Flow Governing Equation } & 27\end{array}$

3 Finite Element Method $\quad 30$

$\begin{array}{ll}3.1 \text { Residual Equations } & 30\end{array}$

3.1.1 Return Mapping Algorithm 33

3.2 Coupled Solution Procedure 36

4 Wellbore Stability Analysis $\quad 38$

$\begin{array}{lll}4.1 & \text { Analytical Solution } & 39\end{array}$

4.2 Numerical Solution $\quad 41$

4.2.1 NR and Accelerated NR $\quad 42$

4.2.2 False Position 44

$5 \quad$ Plugin based Simulator $\quad 46$

5.1 Tectos and Ghem 46

5.2 TopS Data Structure and TopSim Framework 47

5.3 Plugin Scheme $\quad 49$

5.3.1 Main, Driver and Algorithm $\quad 49$

$\begin{array}{lll}5.3 .2 & \text { Reader and Writer } & 50\end{array}$

5.3.3 Analysis, DoFManager and Boundary Condition 51

5.3.4 LinSolver and SparseMatrix $\quad 52$

5.3.5 Other Plugins $\quad 53$

$\begin{array}{lll}5.3 .6 & \text { IElement } & 55\end{array}$

$\begin{array}{lll}5.3 .7 & \text { IMaterial } & 56\end{array}$

$\begin{array}{lll}\text { 5.3.8 Well Stability Scheme } & 58\end{array}$

6 Validation $\quad 62$

6.1 Elastoplastic Cylinder with von Mises Material 62

6.2 Circular hole in a Infinite Mohr Coulomb Material 65

6.3 Oedometric Test 66

$\begin{array}{lll}6.4 & \text { Poroelastic Column } & 70\end{array}$

6.5 Poroelastic Wellbore Problem 72 
$\begin{array}{lll}7 & \text { Case Study } & \mathbf{7 6}\end{array}$

$\begin{array}{lll}7.1 & \text { Sarvak Formation } & 76\end{array}$

$\begin{array}{lll}7.2 & \text { Tensile Stress Validation } & 79\end{array}$

$\begin{array}{lll}7.3 \text { Gulf of Mexico } & 80\end{array}$

7.4 Espírito Santo Basin 83

8 Conclusion $\quad 91$

$\begin{array}{lll}8.1 & \text { Future Works } & 92\end{array}$

$\begin{array}{ll}\text { Bibliography } & 94\end{array}$ 


\section{List of figures}

$\begin{array}{lll}\text { Figure 1.1 General workflow for a wellbore analysis } & 14\end{array}$

$\begin{array}{lll}\text { Figure 1.2 Common instability causes } & 15\end{array}$

$\begin{array}{lll}\text { Figure 1.3 Well failure for different mud weights } & 16\end{array}$

Figure 2.1 Yield surface of the von Mises criterion 22

Figure 2.2 Yield surface of the MC criterion 23

Figure 2.3 Yield surface of the MCC criterion 25

Figure 2.4 Yield surface of the MCC criterion in the principal stress

space with the hydrostatic pressure axis as a blue dashed line $\quad 26$

$\begin{array}{lll}\text { Figure 2.5 Virgin compression line and swelling line } & 27\end{array}$

Figure 4.1 Example of a safe mud pressure window 38

Figure 5.1 Tectos geomechanical attribute tab 46

Figure 5.2 Example of Lua configuration file with the required plugins. 48

$\begin{array}{lll}\text { Figure 5.3 Plugin scheme for a mechanical analysis } & 54\end{array}$

Figure 5.4 Plugin Manager function calls to request elements and materials $\quad 55$

Figure 5.5 Element plugin scheme in a mechanical analysis $\quad 56$

Figure 5.6 Material scheme for a VM material $\quad 57$

Figure 5.7 Plugin scheme to solve a well stability problem 59

Figure 5.8 Simulation scheme in a coupled simulation 60

Figure 5.9 Element scheme in a coupled simulation 61

$\begin{array}{lll}\text { Figure 6.1 Cross-section of the elastoplastic cylinder } & 62\end{array}$

$\begin{array}{lll}\text { Figure 6.2 Quarter cylinder } & 64\end{array}$

Figure 6.3 Stresses vs radial coordinate, $75 \mathrm{MPa} \quad 64$

a Radial stress 64

b Hoop stress 64

Figure 6.4 Stresses vs radial coordinate, $180 \mathrm{MPa}$

a Radial stress 64

b Hoop stress 64

Figure 6.5 Cylindrical hole in a Mohr Coulomb material 65

Figure 6.6 Stresses vs radial coordinate 66

a Radial stress 66

b Hoop stress 66

$\begin{array}{lll}\text { Figure } 6.7 & \text { Oedometer test model } & 67\end{array}$

Figure 6.8 Vertical displacement vs \# Loadsteps 68

Figure 6.9 Strains vs \# Loadsteps 69

a Total vertical strain 69

b Plastic vertical strain $\quad 69$

Figure 6.10 Variation of the Young modulus and relation lnP-specific volume of the throughout the simulation $\quad 69$

a Young Modulus $\quad 69$

b $\quad \ln \mathrm{P}$ vs specific volume $\quad 69$ 
Figure 6.11 Stress path and yield surface in the P x q space $\quad 69$

Figure 6.12 Poroelastic column model $\quad 70$

Figure 6.13 Pore pressure distribution in the poroelastic column $\quad 72$

a Pore pressure at the bottom of the column during the simulation $\quad 72$

b Pore pressure along the middle of the column at 10s $\quad 72$

Figure 6.14 Wellbore model 73

Figure 6.15 Total stresses vs radial distance from the center, after $0.1 \mathrm{~s} 75$

a Horizontal stresses $\quad 75$

b Vertical stresses $\quad 75$

Figure 6.16 Shear stress and Pore pressure vs radial distance from the center, after $0.1 \mathrm{~s} \quad 75$

a Shear stress $\quad 75$

b Pore pressure $\quad 75$

$\begin{array}{lll}\text { Figure 7.1 Sarvak case study model } & 76\end{array}$

a FE model for the Sarvak case study $\quad 76$

b Zoom at the borehole region $\quad 76$

Figure 7.2 Yielded area for the Sarvak case study at the final $\begin{array}{ll}\text { internal pressure. } & 78\end{array}$

Figure 7.3 Maximum principal effective stress at the end of the $\begin{array}{ll}\text { analysis } & 81\end{array}$

Figure 7.4 Permeability vs Porosity trend $\quad 82$

Figure 7.5 FE model from the seismic image 84

$\begin{array}{lll}\text { Figure 7.6 VM equivalent stress after a hundred years } & 86\end{array}$

Figure 7.7 $\quad$ Yielded zone after a hundred years $\quad 86$

$\begin{array}{lll}\text { Figure 7.8 } & \text { Mud pressure window } & 89\end{array}$

Figure 7.9 Zoom at the wellbore section depth 90 


\section{List of tables}

Table 4.1 Minimum allowable internal pressure for each possible stress regime using $\mathrm{MC}$ criterion

Table 4.2 Maximum allowable internal pressure for each possible stress regime using $\mathrm{MC}$ criterion

Table 6.1 Parameters for the cylinder model 63

Table 6.2 Parameters for the MC hole model 66

Table 6.3 Parameters and loads for the MCC hole model 67

Table 6.4 Parameters and loads for the poroelastic column model $\quad 71$

Table 6.5 Parameters and loads for the well model 74

$\begin{array}{lll}\text { Table 7.1 } & \text { Parameters and loads for the Sarvak case study } & 77\end{array}$

Table 7.2 Stability analysis parameters for the Sarvak case study $\quad 77$

Table 7.3 Results for the Sarvak case study 78

Table 7.4 Parameters and loads for the tensile failure case study 79

Table 7.5 Stability analysis parameters for the tensile failure case study $\quad 80$

$\begin{array}{lll}\text { Table 7.6 } & \text { Results for the tensile failure case study } & 80\end{array}$

Table 7.7 Parameters and loads for the GoM case study 82

Table 7.8 Stability analysis parameters for the GoM case study 83

Table 7.9 Results for the GoM case study 83

Table 7.10 MCC criterion parameters $\quad 85$

Table 7.11 MC criterion parameters $\quad 85$

Table 7.12 Parameters and loads for the wellbore section 87

Table 7.13 Stability analysis parameters for the wellbore stability analysis $\quad 88$

Table 7.14 Results for the lower limit of the mud pressure window 88

Table 7.15 Results for the upper limit of the mud pressure window 88 


\section{List of Abreviations}

API - Application Programming Interface

CPA - Cutting Plane Algorithm

CPP - Closest Point Projection

CPU - Central Processing Unit

DM - Double Mechanism

DOF - Degree of Freedom

FEM - Finite Element Method

FP - False Position

LWD - Logging While Drilling

MC - Mohr-Coulomb

MCC - Modified Cam-Clay

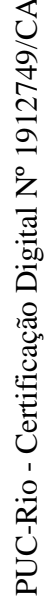

MGEO - Geologichal Modelling

MWD - Measurement While Drilling

NR - Newton-Raphson

PCG - Preconditioned Conjugate Gradient

TVD - True Vertical Depth

VM - von Mises 


\section{Introduction}

\section{1}

\section{Motivation}

Well stability problems are one of the major challenges faced by the oil and gas industry and continues to be an issue as deepwater exploration grows. Accidents related to well stability issues usually end up being the main factor in the nonproductive time during drilling, accounting for $5.6 \%$ and $12.6 \%$ of the total drilling time for nonsubsalt and subsalt on deepwater wells, respectively [1]. Therefore, the use of newer methods and technologies is essential to guarantee a viable exploration of what was previously a high-risk project.

Wells are susceptible to instability issues during the drilling phase, but it may also take place during the production stage when depletion begins or a secondary recovery is initiated [2]. These issues are usually a product of multiple factors involving geomechanical characteristics of the rock formation, well trajectory and even the techniques and equipment used to drill and operate the well. The stability analysis must consider all the different factors involved, which frequently results in complex analysis that require computational assistance or even simplifications to become feasible.

The well life cycle may be divided into a few steps. First, before any actual drilling operation starts, an exploration team studies different sites to find potential new reservoirs and gathers the initial data about the physical condition of the region. Then, as the possible locations are determined, an exploratory drilling step begins, opening the first wellbores to verify the presence of hydrocarbon, as well as acquire more information of the surrounding region with logging while drilling (LWD), measurement while drilling (MWD) and seismic data processing techniques, to cite a few. After the exploratory drilling ends, the operation enters an appraisal step to quantify the volume of the reservoir. If the verdict is favorable, the project enters the development and production step until all the economically viable reserves deplete. In the end, all wells are decommissioned, removing all installations and plugging the existing wells [3]. 
During both the exploratory and appraisal phases, all drilled wellbores must be deemed safe, to avoid technical and environmental accidents. Figure 1.1 shows a regular workflow used for wellbore analyses [4]. The initial data gathered is used to estimate the material parameters, while the in-situ stresses and pore pressure are used to compute the wellbore stresses. Then, the loads and resistances are compared to an adequate failure criterion to create a model to predict wellbore response. This model is continuously updated as new information from drilling events and new logs are acquired until it stops requiring further calibration. The model can then be used to plan new wells in the region, to perform real-time updates during drilling operations or even to reestimate previously measured in-situ stresses and rock properties.

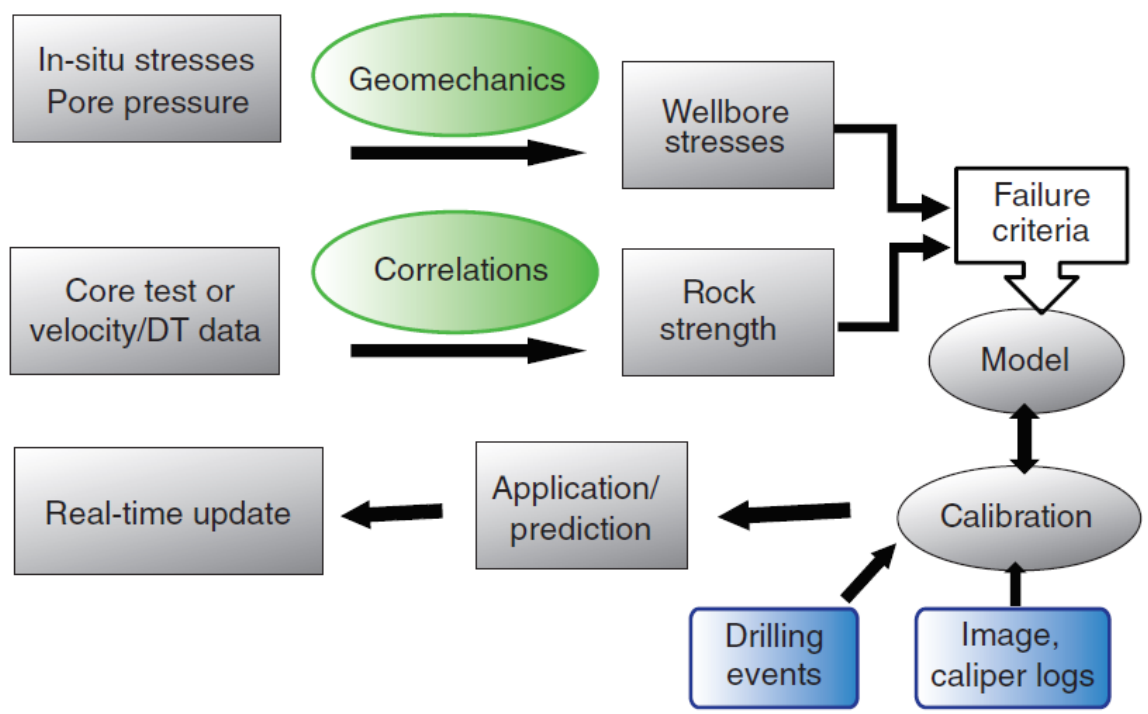

Figure 1.1: General workflow for a wellbore analysis [4]

\section{2}

\section{Common Issues}

Figure 1.2 summarizes common causes of wellbore instability and divides them into uncontrollable and controllable factors. Uncontrollable factors are mostly related to the formation process of the region, where an unfavorable setting appears, such as a high-stress field or an abnormal pore pressure field. On the other hand, the controllable factors are related to variables in the drilling process and are influenced by the uncontrollable factors. Therefore, after the uncontrollable factors are discovered and modeled, a wellbore stability analysis is required to determine the remaining variables in the drilling operation.

Among all controllable factors listed in Figure 1.2, the bottom hole pressure plays a crucial role in the wellbore stability. When the material is 


\begin{tabular}{||c|c||}
\hline \multicolumn{2}{|c|}{ Causes of Wellbore Instability } \\
\hline Uncontrollable (Natural) Factors & Controllable Factors \\
\hline Naturally Fractured or Faulted Formations & Bottom Hole Pressure (Mud Density) \\
\hline Tectonically Stressed Formations & Well Inclination and Azimuth \\
\hline High In-situ Stresses & Transient Pore Pressures \\
\hline Mobile Formations & Physico/chemical Rock-Fluid Interaction \\
\hline Unconsolidated Formations & Drill String Vibrations \\
\hline Naturally Over-Pressured Shale Collapse & Erosion \\
\hline Induced Over-Pressured Shale Collapse & Temperature \\
\hline
\end{tabular}

Figure 1.2: Common instability causes [5]

removed during the perforation, the drilling mud pressure becomes responsible for balancing the pre-existent wellbore stresses. This pressure is the result of the chosen mud weight - or equivalent circulating density if pressure drops are to be considered -, which is dependent on the mud composition. Other factors, such as well inclination, in situ stress orientation and physicochemical interactions between the mud and the medium, also influence the final weight (and composition) required for the mud.

The final mud weight and pressure are directly related to the existent in situ stresses, pore pressure and rock resistance. Figure 1.3 shows an example of the relationship between the mud weight and the other existing loads. The minimum mud pressure (or weight) is determined by the pore pressure and the shear resistance of the material (or the pore pressure and shear failure gradients). If the pressure is lower than either of these values, wellbore washouts/fluid kicks may occur or the wellbore may experience a breakout or an excessive elliptical enlargement. As the mud pressure increases, the breakout width reduces, at the risk of approaching the fracture pressure (or fracture gradient). Applying a pressure higher than the fracture pressure may lead to the initiation or propagation of fractures in the formation. Any further increase in the mud pressure results in the loss of mud circulation until it is entirely lost $[4,6]$.

As for the remaining controllable factors, other studies in the literature discuss different approaches to each one of them. For instance, a semi-analytical algorithm was developed in [7] to generate an optimal well path according to the analytical solution to the optimal direction of a well [8], whereas two different nonlinear models were proposed in [9] and [10] to reproduce the drill string vibrations and the authors in [11] discuss the effects of the physicochemical effects on the wellbore stability analysis. Each one of these factors will impact the mud weight stability window and must be considered 
in a complete wellbore stability analysis.

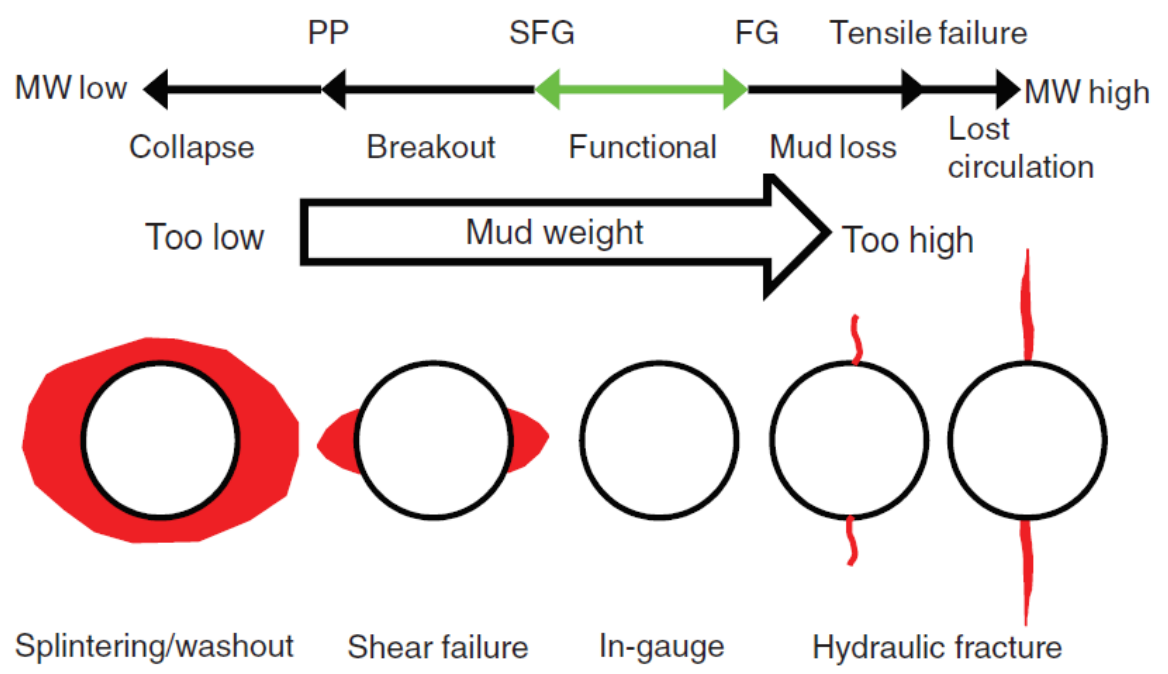

Figure 1.3: Well failure for different mud weights [4]

\section{3}

\section{Objectives}

The main goal of this work is to develop a modular simulator to perform wellbore stability analyses and to compute the optimal mud pressure window. In particular, the specific objectives at the end of this work are:

- Develop a flexible and easily extensible simulator using a plugin architecture;

- Test different methods to compute the mud pressure window;

- Solve wellbore stability problems based on case studies from the literature.

\section{4}

\section{Outline}

The remainder of this thesis is divided as follows. Chapter 2 introduces the theoretical formulation to model the physical behavior of the porous medium studied here, showing both the mechanical equilibrium and the single-phase fluid flow equations. Chapter 3 explains all numerical methods implemented in this work, such as the Finite Element Method, algorithms to solve the plasticity problem and different strategies to solve the coupled system. Chapter 4 outlines the methodology used here and a more traditional approach to mud pressure calculation. In Chapter 5, we present the proposed modular simulator, based on the plugin framework developed at Tecgraf/PUC-Rio, as 
well as other software and resources needed for the simulator to work. Chapters 6 and 7 presents, respectively, several analyses to validate the simulator and to demonstrate a possible application in real-world problems. Finally, Chapter 8 concludes the present work, highlighting possible options for future works. 


\section{Theory}

The goal of this Chapter is to present the main equations in the geomechanical modeling of a deformable porous medium. Most of the theory discussed next is taken from [12] and [13], where Biot's theory describes the interaction between the total stresses, effective stresses and pore pressure in the porous medium. Tensile stresses are assumed positive and compressive stresses are negative in the solid phase of the porous medium and pore pressure is positive in the fluid phase. The porous medium is assumed fully saturated with a single-phase Newtonian fluid and its state is defined by the displacement field $\boldsymbol{u}$ and pore pressure $p$. Throughout this work, bold lower-case letters and bold upper-case letters represent vectors and matrices, respectively, and both stresses and strains are expressed as vectors unless stated otherwise.

\section{1}

\section{Mechanical Equilibrium}

The mechanical equilibrium equation of a porous medium derives from the Principle of Virtual Work in Equation (2-1), assuming quasi-static conditions, for a body $\Omega$ with boundary $\partial \Omega=\Gamma_{u} \cup \Gamma_{t}$. The variation of the functional that describes the problem is

$$
\begin{gathered}
\delta \Pi=\int_{\Omega} \delta \boldsymbol{\varepsilon}^{T} \dot{\boldsymbol{\sigma}} d \Omega-\int_{\Omega} \delta \boldsymbol{u}^{T} \dot{\boldsymbol{b}} d \Omega-\int_{\Gamma} \delta \boldsymbol{u}^{T} \dot{\boldsymbol{t}} d \Gamma=0, \\
\boldsymbol{u}=\boldsymbol{u}^{*} \text { on } \Gamma_{u} \\
\boldsymbol{T} \dot{\boldsymbol{\sigma}}=\dot{\boldsymbol{t}} \text { on } \Gamma_{t}
\end{gathered}
$$

where the volume $\Omega$ is subjected to the rate of body forces $\dot{\boldsymbol{b}}$, prescribed displacement $\boldsymbol{u}^{*}$ on $\Gamma_{u}$ and rate of surface forces $\boldsymbol{t}$ on boundary $\Gamma_{t}$ opposed by the internal reaction $\boldsymbol{T} \dot{\boldsymbol{\sigma}}$, where $\dot{\boldsymbol{\sigma}}$ is the rate of total stresses and $\boldsymbol{T}$ is a matrix related to a unit vector normal to the surface $\Gamma_{t}$. All terms of Equation (2-1) are multiplied by the corresponding variations of the displacement field $\boldsymbol{u}$ and total strains $\boldsymbol{\varepsilon}$.

Imposing an additive decomposition of strains, $\boldsymbol{\varepsilon}$ becomes

$$
\varepsilon=\varepsilon_{e}+\varepsilon_{p}+\varepsilon_{c}
$$


where the terms on the right hand side are respectively the elastic strains, plastic strains and an aggregation of all other possible strains from other physical effects (thermal, viscous, chemical interactions, etc). In this work, the contributions summarized in $\varepsilon_{c}$ are disregarded.

In a porous medium, the rate of total stresses $\dot{\boldsymbol{\sigma}}$ is

$$
\dot{\boldsymbol{\sigma}}=\dot{\boldsymbol{\sigma}}^{\prime \prime}-\alpha \boldsymbol{m} \dot{p}
$$

where $\dot{\boldsymbol{\sigma}}^{\prime \prime}$ is the rate of effective stresses acting on the solid skeleton, $\dot{p}$ is the rate of pore pressure in the porous medium, $\alpha$ is the Biot's coefficient, evaluated from the ratio between the bulk modulus of the medium and the solid skeleton, Equation (2-4), and $\boldsymbol{m}$ is a vector with 1 in the normal directions of the stresses and 0 on the shear components.

$$
\alpha=1-\frac{K}{K_{s}}
$$

The total strains acting on the body are assumed be infinitesimal. Therefore, the compatibility relations between strains and displacements are given by the differential operator $\boldsymbol{L}$,

$$
\boldsymbol{\varepsilon}=\boldsymbol{L} \boldsymbol{u}, \boldsymbol{L}=\left[\begin{array}{ccc}
\frac{\partial}{\partial x} & 0 & 0 \\
0 & \frac{\partial}{\partial y} & 0 \\
0 & 0 & \frac{\partial}{\partial z} \\
\frac{\partial}{\partial y} & \frac{\partial}{\partial x} & 0 \\
\frac{\partial}{\partial z} & 0 & \frac{\partial}{\partial x} \\
0 & \frac{\partial}{\partial z} & \frac{\partial}{\partial y}
\end{array}\right],
$$

in the 3D case. The material is assumed, at first, isotropic and linear elastic as a simplification, although rocks often show both nonlinear elastic behavior and anisotropy. The constitutive equation is then given by

$$
\dot{\boldsymbol{\sigma}}^{\prime \prime}=\boldsymbol{D}_{e} \dot{\varepsilon}_{e}=\boldsymbol{D}_{e p} \dot{\varepsilon},
$$

with $\boldsymbol{D}_{\boldsymbol{e}}$ as the elastic tangent operator, defined in Table 4.3 of [12] for different problem conditions, and $\boldsymbol{D}_{\boldsymbol{e}}$ as the elastoplastic tangent operator, discussed later. For now, the operator will be referred to as $\boldsymbol{D}_{\boldsymbol{T}}$, meaning the elastic or elastoplastic operator according to the current material state.

Substituting all previous Equations in (2-1), Equation (2-1) becomes

$$
\delta \Pi=\int_{\Omega} \delta \boldsymbol{u}^{T} \boldsymbol{L}^{T} \dot{\boldsymbol{\sigma}}^{\prime \prime} d \Omega-\int_{\Omega} \alpha \delta \boldsymbol{u}^{T} \boldsymbol{L}^{T} \boldsymbol{m} \dot{p} d \Omega-\int_{\Omega} \delta \boldsymbol{u}^{T} \dot{\boldsymbol{b}} d \Omega-\int_{\Gamma} \delta \boldsymbol{u}^{T} \dot{\boldsymbol{t}} d \Gamma=0
$$

In this work, the material is assumed to be under plane strain conditions. Hence, all out-of-plane total strain components and out-of-plane shear stresses 
remain constant. The operators $\boldsymbol{L}$ and $\boldsymbol{D}_{T}$ retain only the components related to $\varepsilon_{x x}, \varepsilon_{y y}, \varepsilon_{x y}$, their respective stresses and the pair $\varepsilon_{z z, e}$ and $\sigma_{z z}$ is used to evaluate if yielding occurs in the new stress state.

\subsection{1}

\section{Yield Criteria}

Even though Equation (2-7) describes the global equilibrium of the problem, the local yield problem still needs to be addressed. Materials can only sustain elastic (reversible) deformation up to a limit, after which it begins to develop irreversible plastic deformations. A yield function models this transition to the plastic regime. When it reaches zero, the material starts to yield. Thus, an elastic domain is defined as [14]

$$
\mathcal{E}=\{\boldsymbol{\sigma} \mid \Phi(\boldsymbol{\sigma}, \boldsymbol{A})<0\},
$$

where it contains all possible states of stresses $\boldsymbol{\sigma}$ and hardening forces $\boldsymbol{A}$ such that the material only suffers elastic deformations. Therefore, the yield function defines a yield surface, or hypersurface, in the stress space. Note that $\boldsymbol{\sigma}$ corresponds to the effective stresses, but the symbol " was dropped here for simplicity.

When the current state of the material reaches a critical value $(\Phi(\boldsymbol{\sigma}, \boldsymbol{A})=$ $0)$, it starts to yield. If stressed any further, the material develops plastic strains and a variation in its internal variables $\alpha$, a set of variables that describes the hardening or softening behavior [14]. Their evolution is given by

$$
\dot{\boldsymbol{\varepsilon}}_{p}=\dot{\gamma} \boldsymbol{N}=\dot{\gamma} \frac{\partial \Psi}{\partial \boldsymbol{\sigma}}=\dot{\gamma} \frac{\partial \Phi}{\partial \boldsymbol{\sigma}}
$$

and

$$
\dot{\alpha}=\dot{\gamma} \boldsymbol{H}=-\dot{\gamma} \frac{\partial \Psi}{\partial \boldsymbol{A}}=-\dot{\gamma} \frac{\partial \Phi}{\partial \boldsymbol{A}},
$$

where $\dot{\gamma}$ is the plastic multiplier, a nonnegative scalar, $\boldsymbol{N}$ and $\boldsymbol{H}$ represent the derivatives of the potential function with respect to the stresses and hardening forces. Equations (2-9) and (2-10) are the plastic flow rule and the hardening law. Here, it is assumed that both are associative, i.e., the plastic potential $\Psi$ is equal to $\Phi$.

Equations (2-9) and (2-10) together with the additive decomposition of strains (2-2), the constitutive relations in (2-6) and the conditions (2-11), (2-12) and (2-13), i.e.

$$
\begin{gathered}
\Phi \leq 0, \\
\dot{\gamma} \Phi=0,
\end{gathered}
$$


and

$$
\dot{\gamma} \geq 0
$$

define the basic aspects of a general elastoplastic model [14].

The complementary condition

$$
\dot{\gamma} \dot{\Phi}=0
$$

is introduced to write an expression for the elastoplastic tangent operator. If the material is still in the elastic domain, $\gamma$ remains constant $(\dot{\gamma}=0)$. When it starts to yield all stress states must obey $\Phi=0$ and therefore

$$
\dot{\Phi}=\frac{\partial \Phi}{\partial \boldsymbol{\sigma}} \dot{\boldsymbol{\sigma}}+\frac{\partial \Phi}{\partial \boldsymbol{A}} \dot{\boldsymbol{A}}=\overline{\boldsymbol{N}}^{T} \dot{\boldsymbol{\sigma}}+\overline{\boldsymbol{H}}^{T} \dot{\boldsymbol{A}}=0 .
$$

Substituting (2-2), (2-6) and

$$
\dot{\boldsymbol{A}}=\frac{\partial \boldsymbol{A}}{\partial \boldsymbol{\alpha}} \dot{\boldsymbol{\alpha}}=\boldsymbol{G} \dot{\boldsymbol{\alpha}}
$$

in $(2-15), \dot{\gamma}$ can be written as

$$
\dot{\gamma}=\frac{\overline{\boldsymbol{N}}^{T} \boldsymbol{D}_{e} \dot{\varepsilon}}{\overline{\boldsymbol{N}}^{T} \boldsymbol{D}_{e} \boldsymbol{N}-\overline{\boldsymbol{H}}^{T} \boldsymbol{G H}} .
$$

The last step is to substitute (2-17), (2-2) and (2-9) in (2-6). The elastoplastic tangent operator becomes

$$
\boldsymbol{D}_{e p}=\boldsymbol{D}_{e}-\frac{\left(\boldsymbol{D}_{e} \boldsymbol{N}\right)\left(\boldsymbol{D}_{e} \overline{\boldsymbol{N}}\right)^{T}}{\overline{\boldsymbol{N}}^{T} \boldsymbol{D}_{e} \boldsymbol{N}-\overline{\boldsymbol{H}}^{T} \boldsymbol{G} \boldsymbol{H}}
$$

The operator defined in Equation (2-18) is also referred to as the continuum elastoplastic tangent operator.

After defining a yield model, the next step is to solve the elastoplastic problem with a suitable algorithm to properly evaluate the plastic multiplier, plastic strains and the other necessary variables. Chapter 3 discusses specific algorithms to solve the plasticity problem. The reader is referred to [14], [15] and [16] for a more in-depth description of the basic theory of plasticity.

\subsubsection{1}

\section{von Mises}

The von Mises (VM) criterion was proposed in [17]. It relates the uniaxial yield strength of a material, $\sigma_{Y}$, measured in a traction test, to the second invariant of the deviatoric stress tensor, $\mathrm{J}_{2}$. Mathematically, the perfectly plastic version of the yield criterion is

$$
\Phi=\sqrt{3 J_{2}}-\sigma_{Y}=\sqrt{\frac{\left(\sigma_{1}-\sigma_{2}\right)^{2}+\left(\sigma_{1}-\sigma_{3}\right)^{2}+\left(\sigma_{2}-\sigma_{3}\right)^{2}}{2}}-\sigma_{Y} .
$$


Physically, it represents a correlation for the maximum allowable deviatoric energy stored in a material before yielding, as it is insensitive to the mean stress. This characteristic is also present in the graphical representation of the function in the principal stress space, illustrated in Figure 2.1. The surface becomes an infinite cylinder, with its axis aligned with the mean stress axis.

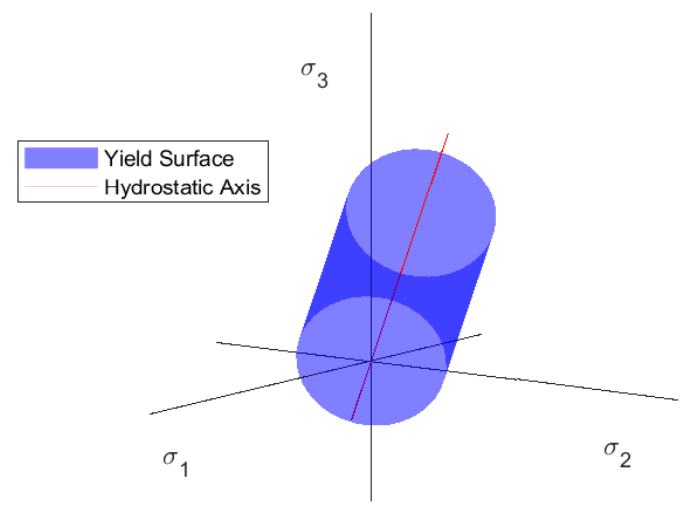

Figure 2.1: Yield surface of the von Mises criterion

\subsubsection{2 \\ Mohr-Coulomb}

In the Mohr-Coulomb (MC) criterion, Mohr proposed that the failure would be determined by the larges Mohr's circle acting on a point and consequently controlled by $\sigma_{1}$ and $\sigma_{3}$. On the other hand, Coulomb suggested that failure would occur when a combination of the normal and shear stresses, $\sigma_{n}$ and $\tau_{n}$, respectively, acting on a specific plane reached a critical value [18]. Two material parameters, the cohesion, $c$, and the angle of internal friction, $\phi$, determine the critical value. The yield function is written as

$$
\tau_{n}=c-\sigma_{n} \tan \phi
$$

or

$$
\frac{\sigma_{1}-\sigma_{3}}{2} \cos \phi=c \cos \phi-\frac{\sigma_{1}+\sigma_{3}}{2} \sin \phi,
$$

in terms of the principal stresses. Unlike the VM criterion, Equation (2-21) leads to a pressure-sensitive behavior, commonly found in soils, rocks and concrete. 
Equation (2-21) can also be cast into an invariant representation, in Equation (2-22), which is more suited to numerical simulations [15]. The yield function now depends on the Lode angle $\theta$, defined in Equation (2-23), and indirectly on the third invariant of the deviatoric stress tensor $J_{3}$, i.e.

$$
\begin{gathered}
\Phi=\left(\cos \theta-\frac{1}{\sqrt{3}} \sin \theta \sin \phi\right) \sqrt{J_{2}}+\frac{I_{1}}{3} \sin \phi-c \cos \phi \\
\theta=\frac{1}{3} \sin ^{-1}\left(\frac{-3 \sqrt{3} J_{3}}{2 J_{2}^{\frac{3}{2}}}\right),-\frac{\pi}{6} \leq \theta \leq \frac{\pi}{6} .
\end{gathered}
$$

Even though the MC criterion is one of the most used in the literature to model the elastoplastic behavior of rocks, it still has some major disadvantages. Firstly, its yield surface is a hexagonal pyramid, as shown in Figure 2.2, which results in nondifferentiable points in respect to the stresses on the apex and along the edges that pass through the apex. To solve the differentiability issue, all edges are rounded off following a procedure described in [15]: directly evaluate Equation (2-22) on $\theta= \pm \frac{\pi}{6}$ before differentiating the Equation. As for the apex, a hyperbolic approximation is employed [19]. The "smoothened" equation becomes

$$
\Phi=\sqrt{\left(\cos \theta-\frac{1}{\sqrt{3}} \sin \theta \sin \phi\right)^{2} J_{2}+a^{2} \sin ^{2} \phi}+\frac{I_{1}}{3} \sin \phi-c \cos \phi,
$$

where $a$ is the semi major axis of the hyperbola measured from the apex of the yield surface in the $\left(\sigma_{n}, \tau_{n}\right)$ space.

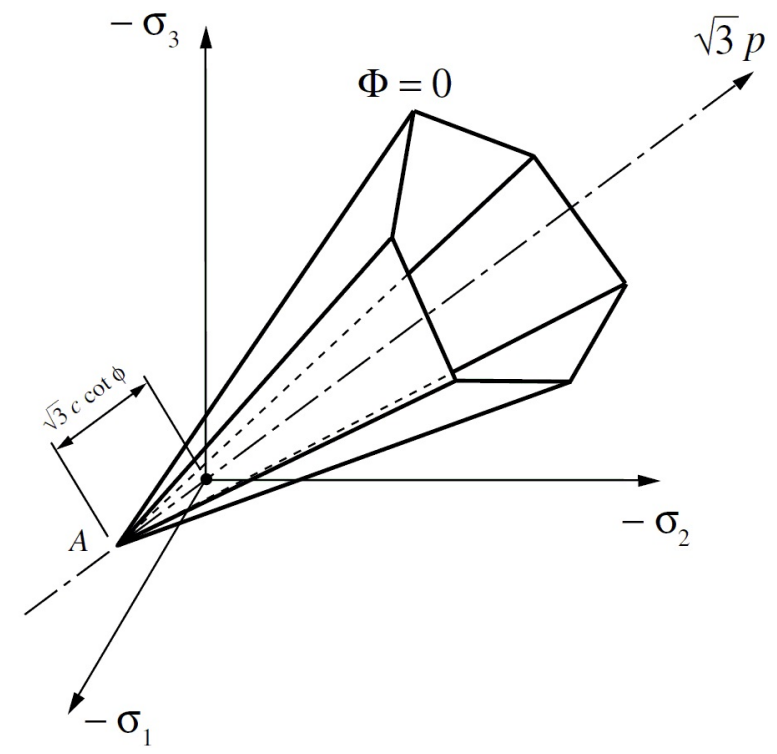

Figure 2.2: Yield surface of the MC criterion, from [14]

Another known issue is related to conservative predictions when using the 
criterion [20,21]. Despite recognizing the role of compressive stresses on the plastic behavior, Equation (2-21) implies that the mean principal stress does not influence the material response. Additionally, for an associative plastic potential, Equation (2-21) leads to dilatant plastic deformations, which are often too excessive [14].

Different modifications are present in the literature for the MC function to solve the differentiability issue. For instance, the Drucker-Prager criterion may be used to avoid the nondifferentiable edges of the yield surface. It consists of a modification of the VM equation to account for the effects of the hydrostatic component of the stress tensor on material strength, adjusted according to different approximations of the MC yield surface [14, 22]. The final yield surface is a cone in the principal stress state, leaving only the apex as a nondifferentiable point. A second option is to use C1 and C2 approximations of the $\mathrm{MC}$ criterion when the current stress state is close to an edge [23, 24, 25]. A final option is to use a multiplanar representation of the MC criterion, along with subdifferentials and a suitable algorithm to solve the plasticity problem $[14,26]$. Other adjustments include the use of nonassociative plastic potential, replacing the friction angle in Equation (2-21) by a smaller one, called the dilatancy angle, or replacing completely the MC criterion by one that explicitly considers the role of the medium principal stress on material strength, such as the Mogi-Coulomb criterion [27].

\subsubsection{3 \\ Modified Cam Clay}

The last yield criterion used in this work is the Modified Cam Clay (MCC), a modification of the original logarithmic Cam Clay. It provides a few advantages when compared to the classical yield functions presented before: it uses a capped yield surface, unlike the previous ones, while also considering the coupled volumetric response of the material when subjected to shear strains up to the Critical State and the nonlinear elastic response through the variation of the bulk modulus according to the hydrostatic stress. The model also requires a small set of physically sound material parameters, which may have contributed to its popularity. However, it is also worth mentioning that the model may be inadequate to predict the behavior of heavy overconsolidated soils [28].

The yield function used for the MCC criterion [14] is expressed as

$$
\Phi=\frac{1}{b^{2}}\left(p-p_{t}+a\right)^{2}+\frac{q^{2}}{M^{2}}-a^{2},
$$

where $p$ is the hydrostatic pressure (not to be confused with the pore pressure), $q$ is the von Mises equivalent stress, $p_{t}$ is the tensile yield hydrostatic stress, $a$ is 
the radius of the elliptical yield surface in the $(p, q)$ space, $M$ is the inclination of the Critical State Line and $b$ is defined as

$$
b=\left\{\begin{array}{l}
1, p \geq p_{t}-a \\
\beta, p<p_{t}-a
\end{array},\right.
$$

with $\beta$ as a material parameter. The corresponding yield surface is represented in Figure 2.3 in the $(p, q)$ space and in Figure 2.4 in the principal stress space.

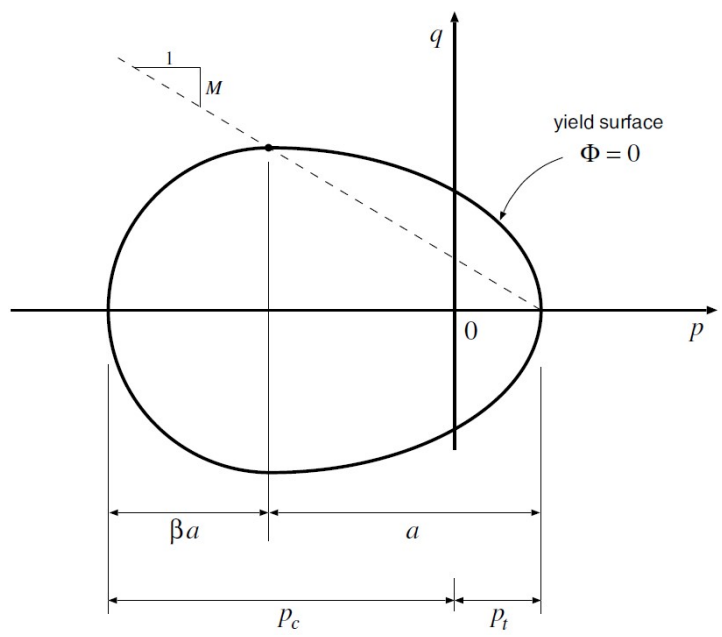

Figure 2.3: Yield surface of the MCC criterion, from [14]

Unlike the original version of the Cam Clay criterion, Equation (2-25) is always differentiable (as long as $\beta>0$ in (2-26)). If the same model is assumed to be associative, the material shows a dilatant volumetric plastic response for a stress state in the subcritical region $\left(p<p_{t}-a\right)$ or a compressive response if it is in the supercritical region $\left(p>p_{t}-a\right)$. Plastic volumetric and deviatoric strains continuously increase during yielding until the pair $(p, q)$ reaches the critical state at the intersection between the critical state line and the yield surface. At that point, the material starts to yield with no volumetric variation.

When using the MCC criterion, it is often assumed that the material also presents variable elastic properties. The variation bulk modulus of the material becomes a function of the hydrostatic pressure, Equation (2-27),

$$
\dot{K}=\frac{v \dot{p}}{\kappa},
$$

where $v$ is the specific volume and $\kappa$ is the slope of the swelling line, illustrated in Figure 2.5. In this situation, the shear modulus or the Poisson coefficient has a fixed value, with all other elastic parameters computed from the pair bulk modulus and shear modulus/Poisson coefficient. The material elastically deforms while following the swelling line, e.g. segment $\overline{c b}$ of Figure 2.5, until it reaches the virgin consolidation line at point b. At this point, it starts to 


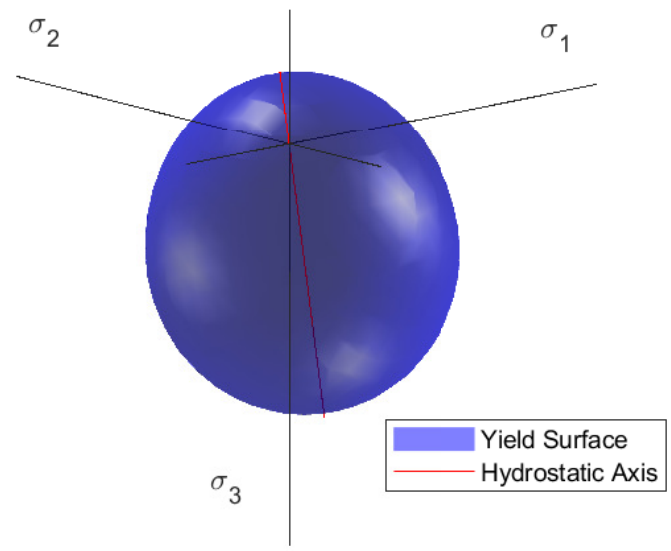

Figure 2.4: Yield surface of the MCC criterion in the principal stress space with the hydrostatic pressure axis as a blue dashed line

develop plastic strains and a new consolidation takes place. If the material is then unloaded, it will deform at a new swelling line, for instance, segment $\overline{e d}$ of Figure 2.5.

A final feature usually present in the MCC model is an exponential isotropic hardening law defined from the slopes of the virgin consolidation line, $\lambda$, and the swelling line. The hardening law becomes [29]:

$$
\dot{a}=a \frac{v}{\lambda-\kappa} \dot{\varepsilon}_{v, p},
$$

representing an increase in the ellipsis radius based on volumetric plastic strain. 


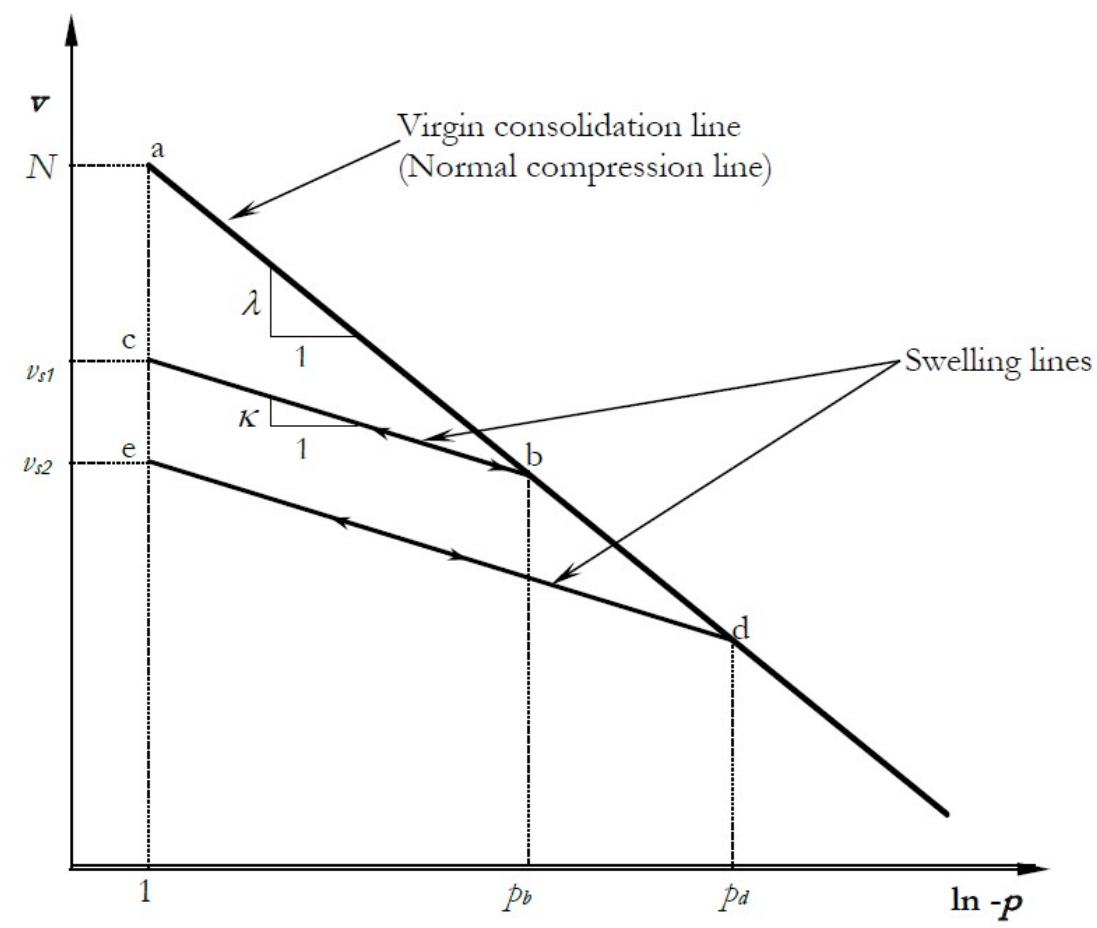

Figure 2.5: Virgin compression line and swelling line, from [30]

\section{2}

\section{Fluid Flow Governing Equation}

The fluid flow governing equations for a fully saturated medium comes from the mass balance equation applied to both solid and liquid phases of a macroscopic volume of a porous medium

$$
\frac{\partial(1-n) \rho^{s}}{\partial t}+\operatorname{div}\left[(1-n) \rho^{s} \boldsymbol{v}^{s}\right]=\frac{(1-n)}{\rho^{s}} \frac{\partial \rho^{s}}{\partial t}-\frac{\partial n}{\partial t}+\operatorname{div}\left[(1-n) \boldsymbol{v}^{s}\right]=0
$$

and

$$
\frac{\partial n}{\partial t}+\frac{n}{\rho^{w}} \frac{\partial \rho^{w}}{\partial t}+\frac{1}{\rho^{w}} \operatorname{div}\left[n \rho^{w} \boldsymbol{v}^{w s}\right]+n \operatorname{div} \boldsymbol{v}^{s}=0,
$$

where $\rho, \boldsymbol{v}$ and $n$ and $\dot{m}$ represent the density of a phase, the velocity of a phase and the porosity of the medium. The single superscript indicates which phase the terms refer to, while the double superscript $w s$ indicates the relative term between the fluid and solid phases. In both equations, the medium undergoes only small deformations.

Two more hypotheses are considered next: the liquid and solid phases are compressible and the fluid transport in the porous medium is a slow phenomenon. The first one implies that 


$$
\frac{1}{\rho^{w}} \frac{\partial \rho^{w}}{\partial t}=\frac{1}{\rho^{w}} \frac{1}{K_{w}} \frac{\partial p}{\partial t},
$$

neglecting thermal effects, where $K_{w}$ is the bulk modulus of the liquid phase, and, for the solid phase,

$$
\frac{1}{\rho^{s}} \frac{\partial \rho^{s}}{\partial t}=\frac{1}{1-n}\left[(\alpha-n) \frac{1}{K_{s}} \frac{\partial p}{\partial t}-(1-\alpha) \operatorname{div} \boldsymbol{v}^{s}\right] .
$$

As for the second hypothesis, a slow transport in a single-phase saturated porous medium allows the application of Darcy's Law, relating the fluid velocity with the pore pressure gradient in the medium and its permeability matrix $\boldsymbol{K}$, together with the fluid's dynamic viscosity $\mu$

$$
\boldsymbol{v}^{w}=\frac{1}{\mu} \boldsymbol{K}\left(-\nabla p+\rho^{w} \boldsymbol{g}\right) .
$$

Adding Equations (2-29) and (2-30), substituting (2-31), (2-32) and (2-33) and replacing the time derivatives with the ${ }^{\cdot}$ symbol, the final equation for the fluid flow in the porous medium becomes

$$
\left(\frac{\alpha-n}{K_{s}}+\frac{n}{K_{w}}\right) \dot{p}+\alpha \operatorname{div} \boldsymbol{v}^{s}+\frac{1}{\rho^{w}} \operatorname{div}\left[\rho^{w} \frac{\boldsymbol{K}}{\mu}\left(-\nabla p+\rho^{w} \boldsymbol{g}\right)\right]=0
$$

The final step to reach the desired governing equation for the fluid flow is, in light of Equation (2-5), to make the substitution

$$
\operatorname{div} \boldsymbol{v}^{s}=\boldsymbol{m}^{T} \dot{\boldsymbol{\varepsilon}}=\boldsymbol{m}^{T} \boldsymbol{L} \dot{\boldsymbol{u}}
$$

and cast (2-34) in its weak form:

$$
\begin{gathered}
\int_{\Omega}\left[-(\nabla \boldsymbol{w})^{T} \frac{\boldsymbol{K}}{\mu}\left(-\nabla p+\rho^{w} \boldsymbol{g}\right)+\boldsymbol{w}^{T} \alpha \boldsymbol{m}^{T} \boldsymbol{L} \dot{\boldsymbol{u}}+\boldsymbol{w}^{T}\left(\frac{\alpha-n}{K_{s}}+\frac{n}{K_{w}}\right) \dot{p}\right] d \Omega+ \\
\int_{\Gamma_{q}} \boldsymbol{w}^{T} \frac{q}{\rho^{w}} d \Gamma=0,
\end{gathered}
$$

with the following boundary conditions for prescribed pore pressure $p^{*}$ on $\Gamma_{p}$ and mass flux $q$ on $\Gamma_{q}$, with direction $\boldsymbol{n}$

$$
\begin{aligned}
p & =p^{*} \text { on } \Gamma_{p}, \\
\rho^{w} \frac{\boldsymbol{K}}{\mu}\left(-\nabla p+\rho^{w} \boldsymbol{g}\right) \cdot \boldsymbol{n} & =q \text { on } \Gamma_{q}, \\
d \Omega=\Gamma & =\Gamma_{p} \cup \Gamma_{q},
\end{aligned}
$$

where $\boldsymbol{w}$ represents an arbitrary set of functions, discussed later in chapter 3. In 
this work, it is assumed that the medium has isotropic constant permeability, even though it may vary spatially, and therefore $\boldsymbol{K}$ becomes a multiple of the identity matrix.

Equations (2-7) and (2-36) and their respective boundary conditions represent the set of governing equations for the problems discussed in this work. The system can be further simplified if it is assumed that the pore pressure field remains constant throughout the analysis or if the material is purely elastic. On the other hand, some simplifications can be removed or changed to consider a more realistic behavior of the soil or rock. For instance, a generalized plane strain state may replace the traditional plane strain state, where the displacement perpendicular to a cross-section is allowed, but the strains do not vary in the direction perpendicular to the cross-section, leading to the representation of a more accurate stress and strain state even in a twodimensional model $[31,32]$. Another example is the use of a dual-porosity field $[32,33,34]$ to more accurately reproduce the behavior of naturally fractured rocks or the use of the Cosserat theory to reproduce the behavior of cemented/non-cemented granular materials [35]. Finally, the model can also consider nonisothermal conditions and viscous behavior, leading to a third governing equation to calculate the temperature field, new terms in Equations (2-7) and (2-36) and local modifications to consider the presence of viscous strains together with the plastic strains. 


\section{Finite Element Method}

\section{1}

\section{Residual Equations}

In order to solve the system of Equations (2-7) and (2-36), the Finite Element Method was used. We start by noting that, since the deformation and fluid flow are slow phenomena, the ${ }^{\cdot}$ symbol is dropped in (2-7), and as a result, the equation becomes:

$$
\delta \Pi=\int_{\Omega} \delta \boldsymbol{u}^{T} \boldsymbol{L}^{T} \boldsymbol{\sigma}^{\prime \prime} d \Omega-\int_{\Omega} \alpha \delta \boldsymbol{u}^{T} \boldsymbol{L}^{T} \boldsymbol{m} p d \Omega-\int_{\Omega} \delta \boldsymbol{u}^{T} \boldsymbol{b} d \Omega-\int_{\Gamma} \delta \boldsymbol{u}^{T} \boldsymbol{t} d \Gamma=0
$$

Next, the body $\Omega$ is discretized into small elements, and the displacement and pore pressure fields are represented by values assigned to each node. Element-wise shape functions combine the nodal data to compute the values at any point. If these functions are Lagrange functions, the nodal values become the nodal displacement $\overline{\boldsymbol{u}}$ and pore pressure $\overline{\boldsymbol{p}}$. The following expressions relate the nodal values and shape function to the field values:

$$
\boldsymbol{u}=\boldsymbol{N}^{u} \overline{\boldsymbol{u}} \quad p=\boldsymbol{N}^{p} \overline{\boldsymbol{p}}
$$

where $\boldsymbol{N}^{u}$ is a number of element nodes by number of degrees of freedom matrix for the displacement field, while $\boldsymbol{N}^{p}$ is a number of element nodes by 1 matrix since there is only one pressure field. In this work, only the linear T3 and Q4 are used with equal order interpolations for both fields. Both elements should give a suitable approximation of the displacements and pore pressure provided that the medium never reaches an undrained limit state. Substituting (3-2) into (3-1) and imposing that the variation of the functional must remain zero for any virtual increment, we obtain the discretized equation the mechanical equilibrium

$$
\int_{\Omega} \boldsymbol{B}^{T} \boldsymbol{\sigma}^{\prime \prime} d \Omega-\int_{\Omega} \alpha \boldsymbol{B}^{T} \boldsymbol{m} \boldsymbol{N}^{p} \overline{\boldsymbol{p}} d \Omega-\boldsymbol{f}^{u}=\boldsymbol{R}^{u}
$$

where the contributions of the body forces, surface loads and any other external forces are summarized in $\boldsymbol{f}^{u} . \boldsymbol{R}^{u}$ represents the residual of the left-hand side 
of (3-3), due to the approximation of the actual displacement field with the Lagrange polynomials. Finally, the $\boldsymbol{B}$ matrix is the result of the product between $\boldsymbol{N}^{u}$ and $\boldsymbol{L}$ and relates the nodal displacements to their respective strains.

A similar procedure derives the final equations for the nodal pore pressures. Employing the Galerkin Method, the shape functions $\boldsymbol{N}^{u}$ and $\boldsymbol{N}^{p}$ replace the weight functions in (2-36):

$$
\begin{gathered}
\int_{\Omega}\left[-\left(\nabla \boldsymbol{N}^{p}\right)^{T} \frac{\boldsymbol{K}}{\mu}\left(-\nabla \boldsymbol{N}^{p} \overline{\boldsymbol{p}}\right)+\boldsymbol{N}^{p T} \alpha \boldsymbol{m}^{T} \boldsymbol{B} \dot{\overline{\boldsymbol{u}}}+\boldsymbol{N}^{p T}\left(\frac{\alpha-n}{K_{s}}+\frac{n}{K_{w}}\right) \boldsymbol{N}^{p} \dot{\overline{\boldsymbol{p}}}\right] d \Omega+ \\
\boldsymbol{f}^{p}=\boldsymbol{R}^{p}
\end{gathered}
$$

where $\boldsymbol{f}^{p}$ is the summation of the gravitational contribution with the mass flow through a surface and, just as in the previous case, $\boldsymbol{R}^{p}$ is the residual of the mass balance equation.

Once (3-3) and (3-4) are provided, the next step is to apply a suitable time integration scheme. Here, the Newmark scheme will be employed to relate the velocities and rate of pore pressure from (3-4) with the displacements and pore pressures at the current time $n$ and the next unknown state $n+1$ after a time step $\Delta t$. The equation becomes [36]:

$$
\dot{\boldsymbol{x}}^{n+1}=\frac{\delta\left(\boldsymbol{x}^{n+1}-\boldsymbol{x}^{n}\right)}{\beta \Delta t}-\left(\frac{\delta}{\beta}-1\right) \dot{\boldsymbol{x}}^{n}
$$

where $\boldsymbol{x}$ represents both displacements or pore pressure and their respective rates when the ${ }^{\prime}$ is applied and $\beta$ and $\delta$ are the Newmark parameters. If $\beta$ and $\delta$ are set to 0.25 and 0.5 , respectively, the scheme will satisfy the necessary conditions for unconditional stability and convergence:

$$
\delta \geq 0.5, \quad \beta \geq 0.25(0.5+\delta)^{2} .
$$

The final step to obtaining the system of algebraic equations for the discretized model is to use the Newton-Raphson (NR) method once (3-5) is substituted in (3-4). The final system is

$$
\left[\begin{array}{cc}
\frac{\partial \boldsymbol{R}^{u}}{\partial \boldsymbol{u}^{n+1}} & \frac{\partial \boldsymbol{R}^{u}}{\partial \boldsymbol{p}^{n+1}} \\
\frac{\partial \boldsymbol{R}^{p}}{\partial \boldsymbol{u}^{n+1}} & \frac{\boldsymbol{\partial} \boldsymbol{R}^{p}}{\partial \boldsymbol{p}^{n+1}}
\end{array}\right]_{i}\left[\begin{array}{c}
\delta \boldsymbol{u} \\
\delta \boldsymbol{p}
\end{array}\right]_{i+1}=-\left[\begin{array}{c}
\boldsymbol{R}^{u} \\
\frac{\beta}{\delta} \Delta t \boldsymbol{R}^{p}
\end{array}\right]_{i+1}^{n+1},\left[\begin{array}{c}
\boldsymbol{u} \\
\boldsymbol{p}
\end{array}\right]_{i}^{n+1}+\left[\begin{array}{c}
\delta \boldsymbol{u} \\
\delta \boldsymbol{p}
\end{array}\right]_{i+1}=\left[\begin{array}{c}
\boldsymbol{u} \\
\boldsymbol{p}
\end{array}\right]_{i+1}^{n+1},
$$

with the overlines on $\boldsymbol{u}$ and $\boldsymbol{p}$ dropped, while the superscripts $n+1$ and $n$ indicate which time the term refers to, the subscript $i+1$ and $i$ refers to the 
iterations of the Newton-Raphson algorithm and

$$
\begin{aligned}
\frac{\partial \boldsymbol{R}^{u}}{\partial \boldsymbol{u}^{n+1}} & =\int_{\Omega} \boldsymbol{B}^{T} \boldsymbol{D}_{T} \boldsymbol{B} d \Omega \\
\frac{\partial \boldsymbol{R}^{p}}{\partial \boldsymbol{p}^{n+1}} & =\int_{\Omega} \frac{\beta}{\delta} \Delta t\left(\nabla \boldsymbol{N}^{p}\right)^{T} \frac{\boldsymbol{K}}{\mu}\left(\nabla \boldsymbol{N}^{p}\right)+\boldsymbol{N}^{p T}\left(\frac{\alpha-n}{K_{s}}+\frac{n}{K_{w}}\right) \boldsymbol{N}^{p} d \Omega \\
-\frac{\boldsymbol{\partial} \boldsymbol{R}^{u}}{\partial \boldsymbol{p}^{n+1}} & =\frac{\partial \boldsymbol{R}^{p}}{\partial \boldsymbol{u}^{n+1}}=\int_{\Omega} \alpha \boldsymbol{B}^{T} \boldsymbol{m} \boldsymbol{N}^{p} d \Omega
\end{aligned}
$$

Equation (3-8a) is the usual stiffness matrix, whereas (3-8b) is the Jacobian matrix of the fluid problem, composed of the permeability and compressibility matrices, respectively, and equation (3-8c) represents the coupling term between the mechanical and fluid systems.

Even though the time integration described in (3-5) is unconditionally stable and convergent and the system presented in (3-8) assumes that the porous medium remains fully saturated at all times, numerical oscillations have been reported throughout the literature when dealing with the system (3-8) and its variations $[37,38,39,40]$. One of the first reports of this phenomenon was in [37], in which the authors noticed the oscillations on the permeable boundaries where loads were applied used the accuracy condition of the consolidation problem to define a minimum initial time step based on the mesh size and the rock properties. In [38], the authors elaborated further into the causes of the numerical oscillations, attributing them to a discretization error and a parabolic variation of the pore pressure field, and proposed a smoothing process to reduce the oscillations in 2 and 3D problems. Then, different error estimates were obtained for the case of discretization of the consolidation problem using the backward Euler method instead of the Newmark method [39]. Finally, a recent work revisited the initial time step requirements to avoid numerical oscillations and derived a new relation between the mesh size and minimum time step to satisfy the Discrete Maximum Principle [40]. Here, the following equation calculates the minimum initial time step for each simulation [40]:

$$
\Delta t \geq \frac{h^{2} \mu}{0.5 k}\left(\frac{\alpha^{2}}{4 D}+\frac{1}{6}\left(\frac{\alpha-n}{K_{s}}+\frac{n}{K_{w}}\right)\right),
$$

where $k$ is the permeability of the medium (one of the diagonal entries of the matrix $\boldsymbol{K}), D$ is the constrained modulus of the rock and $h$ is the element length, computed as the square root of the element area. The initial time step used in all simulations will then be double the largest one computed from all elements in the permeable boundary of the model. 


\subsection{1 \\ Return Mapping Algorithm}

The general scheme to solve Equations (3-3) and (3-4) was described in the previous section. However, a numerical procedure is still needed to locally update the stress state under elastoplastic conditions. In this regard, the plasticity problem is seen as a local problem, solved separately from the global system, given a displacement increment from the global solution. The general idea of the local procedure is discussed next. As a side note, under plane stress conditions, specific modifications are needed in the local problem to keep the global system unchanged, but these differences will not be discussed here $[14,41]$.

This procedure is a simple two-step algorithm. The first step is to assume that the total strain increment computed from the displacement increment, eq. (2-5), is purely elastic. Since all deformations are presumably elastic, Equation (2-6) uses the elastic tangent operator and the current elastic trial strain increment $\varepsilon_{e}^{\text {trial }}$ to calculate a new stress state, called elastic trial stress $\boldsymbol{\sigma}_{e}^{\text {trial }}$, and update the hardening forces and internal variables to elastic trial state. If (2-11) still holds, then the current state remains inside (or at the boundary of) the elastic stress surface. Else, a plastic corrector, or return mapping algorithm, is needed to compute the actual stresses and strains such that conditions (2-11), (2-12) and (2-13) hold [14]. Once the plastic corrector step computes the new corrected stresses and plastic strains, the other remaining properties and attributes of the model are updated and a new iteration of the global NR algorithm begins.

Different plastic corrector schemes have been proposed throughout the literature. The most used schemes are: a group of Closest Point Projection (CPP) methods, based on single-step numerical integration methods that leads to an implicit or explicit system of equations [14, 29, 41], mathematical programming methods, where the plasticity problem becomes a convex optimization problem according to the yield criterion $[42,26]$, and the Cutting Plane Algorithm (CPA), where the consistency condition is explicitly integrated [43]. A less common approach is to use other differential-algebraic equation methods, which leads to more complex systems.

In this work, the Cutting Plane Algorithm (CPA) was chosen as the return mapping algorithm. The algorithm, proposed in [44], is based on a steepest descent strategy [45] and requires only an iterative solution of an explicit equation in the current trial state.

The main idea behind the CPA is to solve the linearized version of the yield condition at the current state. In order to compute the new state at the 
iteration $k+1$ of the algorithm, Equation (2-15) is rewritten in its discretized form $[14]$

$$
\begin{gathered}
\Phi\left(\boldsymbol{\sigma}_{k+1}^{n+1}, \boldsymbol{A}_{k+1}^{n+1}\right)=\Phi\left(\boldsymbol{\sigma}_{k}^{n+1}, \boldsymbol{A}_{k}^{n+1}\right)+\overline{\boldsymbol{N}}^{T} \Delta \boldsymbol{\sigma}^{n+1}+\overline{\boldsymbol{H}}^{T} \Delta \boldsymbol{A}^{n+1}=0 . \\
\Delta \boldsymbol{\sigma}^{n+1}=\boldsymbol{\sigma}_{k+1}^{n+1}-\boldsymbol{\sigma}_{k}^{n+1} \\
\Delta \boldsymbol{A}^{n+1}=\boldsymbol{A}_{k+1}^{n+1}-\boldsymbol{A}_{k}^{n+1}
\end{gathered}
$$

$\Delta \boldsymbol{\sigma}^{n+1}$ and $\Delta \boldsymbol{A}^{n+1}$ are then substituted using the discrete form of eq (2-6)

$$
\Delta \boldsymbol{\sigma}^{n+1}=\boldsymbol{D}_{e} \Delta \varepsilon_{e}^{n+1}
$$

and

$$
\Delta \boldsymbol{A}^{n+1}=\boldsymbol{G} \Delta \boldsymbol{\alpha}^{n+1} .
$$

Using an explicit form of eqs (2-9) and (2-10) and noting that $\dot{\varepsilon}_{p}=-\dot{\varepsilon}_{e}$, the increment of the plastic multiplier can be computed as:

$$
\Delta \gamma=\frac{\Phi\left(\boldsymbol{\sigma}_{k}^{n+1}, \boldsymbol{A}_{k}^{n+1}\right)}{\overline{\boldsymbol{N}}^{T} \boldsymbol{D}_{e} \boldsymbol{N}-\overline{\boldsymbol{H}}^{T} \boldsymbol{G} \boldsymbol{H}}
$$

The pseudo-code for the CPA is presented in Algorithm 1. It requires only simple derivatives of the yield function and the plastic potential with respect to the stresses and hardening forces, which leads to a simple implementation of the algorithm with no need to use third-party implementations to solve the plasticity problem. The simplicity also allows for the use of more complex yield models, where the required derivatives are readily available in general. Besides its simplicity and flexibility, the CPA also presents quadratic convergence rate $[44,46]$.

The consistent tangent operators should also be addressed to use return mapping algorithms in a FE program. In [47], the authors introduced the concept of a consistent elastoplastic tangent operator, which accounts for the change in the stress evaluated from a return mapping algorithm given an infinitesimal strain increment [45]. The continuum operator from (2-18), on the other hand, is derived from the constitutive law and flow rules and does not lead to a consistent scheme. Therefore, to ensure optimal convergence of the global NR algorithm, the consistent operator should be used instead of the continuous operator.

The need for a consistent tangent operator in FE programs represents one of the main drawbacks of the CPA. Unlike CPP methods, with the iterative solution of an implicit function, or in the case of optimization methods, with well-known direct and adjoint methods for differentiation, the CPA solves a 


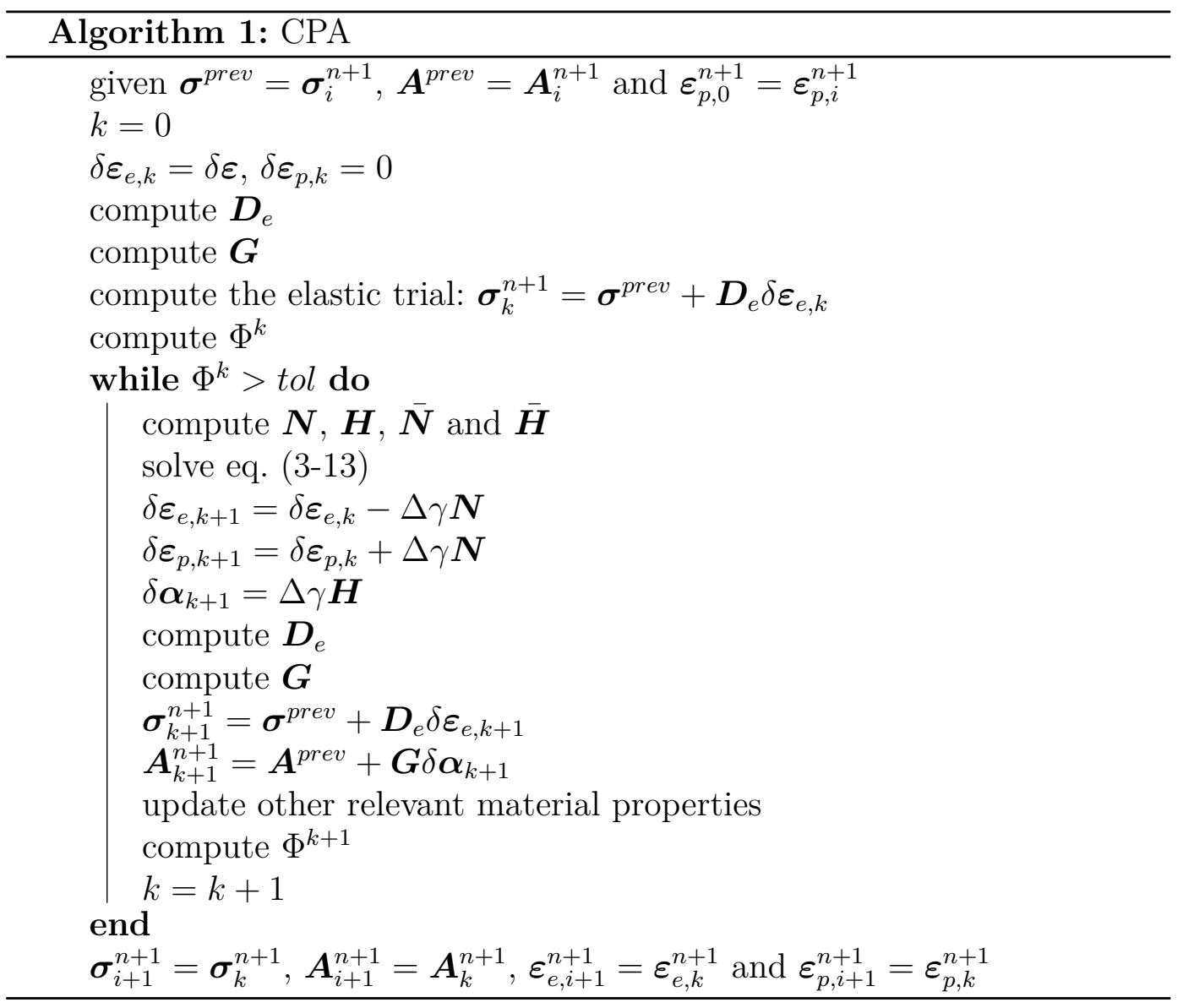

sequence of explicit equations. As a result, the linearization to compute a consistent operator is a complex task, and implementations of the algorithm remain using the continuum operator [14, 43, 45]. Despite the suboptimal convergence with the continuum operator, it was later verified that, as long as the load steps remain relatively small, the continuum operator with the CPA leads to almost identical results similar to other methods with consistent operators [45]. Recently, a novel iterative procedure to compute the operator when using the CPA was derived, but it will not be considered here [43].

Finally, it is worth addressing the use of a variable Young modulus when dealing with the MCC model. Here, an explicit version of equation (2-27) computes the bulk modulus at the known stress state. This treatment is appropriate for the case studies present in the following chapters, where the loads are monotonic. However, if the simulation has cyclic loads, this explicit form of (2-27) may lead to nonconservative behavior, and a tangential modulus should be used [48]. Other variations related to the return mapping equations and plasticity modeling using the MCC model are discussed in a series of contributions by Borja, such as [48] and [49]. 


\section{2}

\section{Coupled Solution Procedure}

With the plasticity problem addressed, the remaining step to solve the mechanical equilibrium and the fluid flow governing equation is the solution of the system (3-7). Two strategies are commonly employed to solve the fully coupled system. The first one is to solve Equation (3-7) with an adequate linear system solver based on the matrix properties. For the governing equations and associative yield criteria considered here, Equations (3-8a) and (3-8b) are symmetric positive definite matrices. Hence, the system (3-7) becomes a symmetric indefinite matrix. Direct solvers exploring the matrix symmetry or iterative unsymmetric solvers, such as the Preconditioned Biconjugate Gradient method, if the mechanical equilibrium equation is multiplied by -1 , are among the options of linear solvers available in this situation.

The second approach to solve the fully coupled problem is to use a staggered solution strategy $[13,42,50,51]$ to solve (3-7). Assume that the system in Equation (3-7) is represented by

$$
\left[\begin{array}{ll}
\boldsymbol{A}_{11} & \boldsymbol{A}_{\mathbf{1 2}} \\
\boldsymbol{A}_{21} & \boldsymbol{A}_{22}
\end{array}\right]_{i}^{n+1}\left[\begin{array}{l}
\boldsymbol{\delta} \boldsymbol{x}_{\mathbf{1}} \\
\boldsymbol{\delta} \boldsymbol{x}_{\mathbf{2}}
\end{array}\right]_{i+1}=\left[\begin{array}{l}
\boldsymbol{F}_{\mathbf{1}}\left(\boldsymbol{x}_{1, i}^{n+1}, \boldsymbol{x}_{2, i}^{n+1}\right) \\
\boldsymbol{F}_{\mathbf{2}}\left(\boldsymbol{x}_{1, i}^{n+1}, \boldsymbol{x}_{2, i}^{n+1}\right)
\end{array}\right] .
$$

Then, by keeping $\boldsymbol{x}_{\mathbf{2}}$ constant, we solve

$$
\boldsymbol{A}_{\mathbf{1 1}, i}^{n+1} \boldsymbol{\delta} \boldsymbol{x}_{\mathbf{1}, i+1}=\boldsymbol{F}_{\mathbf{1}}\left(\boldsymbol{x}_{\mathbf{1}, i}^{n+1}, \boldsymbol{x}_{\mathbf{2}, i}^{n+1}\right)
$$

and update $\boldsymbol{x}_{1}$ using the regular NR scheme. Next, we solve

$$
\boldsymbol{A}_{\mathbf{2} 2, i}^{n+1} \boldsymbol{\delta} \boldsymbol{x}_{\mathbf{2}, i+1}=\boldsymbol{F}_{\mathbf{2}}\left(\boldsymbol{x}_{\mathbf{1}, i}^{n+1}, \boldsymbol{x}_{\mathbf{2}, i}^{n+1}\right),
$$

computing $\boldsymbol{F}_{\mathbf{2}}$ with the updated value of $\boldsymbol{x}_{\mathbf{1}}$. Then, we return to the first equation, with the updated value of $\boldsymbol{x}_{\mathbf{2}}$ in $\boldsymbol{F}_{\mathbf{1}}$ and the process is repeated until $\boldsymbol{x}_{1}$ and $\boldsymbol{x}_{2}$ converge for a given tolerance. With this strategy the coupling nature of the problem is enforced iteratively at every timestep of the solution. The terms $\boldsymbol{A}_{\mathbf{1 1}, i}^{n+1}$ and $\boldsymbol{A}_{\mathbf{2} 2, i}^{n+1}$ in Equations 3-15 and 3-16 correspond to the matrices defined in Equations 3-8a and 3-8b, respectively. Since both systems are now composed of symmetric positive definite matrices, the Preconditioned Conjugate Gradient (PCG) method [52] is used to solve the linear system. Even though the addition of another iterative process may seem computationally expensive, the staggered procedure still presents some benefits over a direct solution of Equation 3-7:

- only mechanical iterations are required since the only source of nonlinearity presented here is the yielding behavior of the medium. The staggered procedure leads to an iterative solution of the mechanical problem fol- 
lowed by the linear flow problem. As long as only a small portion of the model yields, the staggered solution will be more efficient than the coupled solution [42];

- different simulators for the mechanical or fluid problem can be used to solve the coupled problem with only minor modifications [13]. For the plugin simulator, different plugin schemes for both problems are readily combined to perform coupled simulations by providing extra plugins, discussed in Chapter 5;

- the procedure is easily extended to simulate different physical conditions. The single-phase fluid problem can be replaced by a two-phase flow (together with an appropriate linear solver), while the mechanical problem remains the same, or the thermal coupling can be introduced by solving the thermal equations after the displacements and pore pressure increments are available.

The staggered procedure must also be stable to provide an appropriate numerical solution. The scheme presented here, termed drained split, is only conditionally stable according to the von Neumann stability analysis and requires $[51]$

$$
\frac{\alpha^{2}}{D}\left(\frac{\alpha-n}{K_{s}}+\frac{n}{K_{w}}\right)^{-1}<1
$$

where $\alpha$ is the Biot's coefficient, $n$ is the porosity, $K_{s}$ and $K_{w}$ are the solid grain and fluid compressibilities, respectively, and $D$ is the constrained modulus.

Other partitioning strategies can be used to derive different staggered solution schemes, such as the fixed strain split, where the fluid problem is solved first and is also conditionally stable, and the undrained split and fixed stress split, which are unconditionally stable. For more details on other partitioning strategies for the coupled problem, the reader is referred to [51] and [53]. 


\section{4}

\section{Wellbore Stability Analysis}

The goal behind a wellbore stability analysis is to determine the safe mud weight window or mud pressure window. According to the functional window defined in figure 1.3, figure 4.1 shows an example of a mud weight window for a vertical well. In the upper region of the well, the lower limit is determined by the pore pressure, whereas the limit in the lower region is determined by the shear failure. The main challenge of a stability analysis, besides accurately measuring and mapping the properties, pore pressure and loads, is to compute correctly both shear failure and fracture pressure and gradients, as they will depend on both the quality of the data and the available models to reproduce the physical behavior.

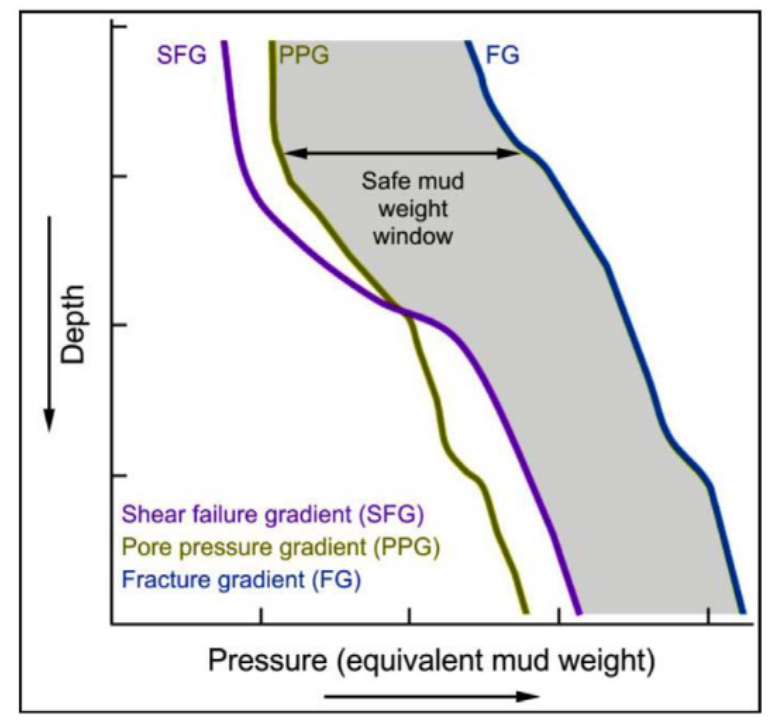

Figure 4.1: Example of a safe mud pressure window [54]

Next, two different approaches to perform a wellbore stability analysis are described. The first one consists of an analytical solution used for specific wellbore projects. The second one is a numerical procedure, which serves as the basis for more complex stability analyses. 


\section{1}

\section{Analytical Solution}

A brief review of wellbore stability models was presented in [55]. In this work, the authors discussed and compared several models, from linear elastic ones to nonlinear constitutive models, considering the influence of fluid flow and anisotropic properties. They also highlighted the importance of an appropriate yield criterion compatible with the formation behavior. The wellbore stability analysis would then follow the traditional steps of a geomechanical analysis:

- Estimation of in situ stresses and pore pressure;

- Estimation of material properties;

- Choice of an adequate constitutive model and yield criterion;

- Computation of the stresses at the wellbore wall;

- Comparison of the wellbore stresses with the chosen failure criterion.

This procedure is general and can be applied theoretically to any wellbore analysis, but could lead to laborious work when used to compute the minimum and maximum internal pressure for a wellbore. Later, this procedure was used to solve the wellbore stability analysis using the $\mathrm{MC}$ criterion [21]. The authors then derived a closed-form solution to compute the mud pressure stability window for vertical wells, which is still used nowadays [20, 56].

The closed-form solution assumes that the medium is fully saturated, but has no poromechanical coupling effects. The stresses at the wellbore wall are computed using the Kirsch solution and then rewritten in the cylindrical coordinate system. The final radial, hoop and axial stresses $\left(\sigma_{r r}, \sigma_{\theta \theta}\right.$ and $\sigma_{z z}$, respectively) are substituted in the $\mathrm{MC}$ criterion. For each combination of $\sigma_{r r}, \sigma_{\theta \theta}$ and $\sigma_{z z}$, indicating the plane that the failure occurs, a breakout or fracture pressure is evaluated. The required breakout (fracture) pressure will be the highest (smallest) pressure from Table 4.1 (or 4.2) and Equations (4-1) to (4-6). In Equations (4-1) to (4-6), $\sigma_{v}, \sigma_{H}$ and $\sigma_{h}$ are the vertical, maximum horizontal and minimum horizontal stresses. As a side note, all compressive stresses and the pore pressure $p$ in both tables and in Equations (4-1) to (4-6) have positive signs, while tensile stresses have negative signs, following the notation from $[21]$.

\begin{tabular}{cc}
\hline Stress Regime & Minimum allowable internal pressure \\
\hline$\sigma_{z z} \geq \sigma_{\theta \theta} \geq \sigma_{r r}$ & $P_{w b 1}=(B-C) / q$ \\
\hline$\sigma_{\theta \theta} \geq \sigma_{z z} \geq \sigma_{r r}$ & $P_{w b 2}=(A-C) /(1+q)$ \\
\hline
\end{tabular}


Continuation of table 4.1

\begin{tabular}{cc}
\hline Stress Regime & Minimum allowable internal pressure \\
\hline$\sigma_{\theta \theta} \geq \sigma_{r r} \geq \sigma_{z z}$ & $P_{w b 3}=A-C-q B$ \\
\hline
\end{tabular}

Table 4.1: Minimum allowable internal pressure for each possible stress regime using MC criterion [21]

\begin{tabular}{cc}
\hline Stress Regime & Maximum allowable internal pressure \\
\hline$\sigma_{r r} \geq \sigma_{\theta \theta} \geq \sigma_{z z}$ & $P_{w f 1}=C+q E$ \\
\hline$\sigma_{r r} \geq \sigma_{z z} \geq \sigma_{\theta \theta}$ & $P_{w f 2}=(C+q D) /(1+q)$ \\
\hline$\sigma_{z z} \geq \sigma_{r r} \geq \sigma_{\theta \theta}$ & $P_{w f 3}=(C-E) / q+D$ \\
\hline
\end{tabular}

Table 4.2: Maximum allowable internal pressure for each possible stress regime using MC criterion [21]

$$
\begin{gathered}
q=\tan ^{2}\left(\frac{\pi}{4}+\frac{\phi}{2}\right) \\
A=3 \sigma_{H}-\sigma_{h} \\
B=\sigma_{v}+2 \nu\left(\sigma_{H}-\sigma_{h}\right) \\
C=2 c \frac{\cos \phi}{1-\sin \phi}-p(q-1) \\
D=3 \sigma_{h}-\sigma_{H} \\
E=\sigma_{v}-2 \nu\left(\sigma_{H}-\sigma_{h}\right)
\end{gathered}
$$

Additionally, when estimating the fracture pressure with the MC criterion, the authors also proposed that a tensile cutoff should be used to address the overestimation of the tensile strength. Since the hoop stress is the first to become tensile on the vertical well, it is directly compared with the tensile strength. Rewriting the hoop stress solution using the in situ stresses and the internal pressure, the final equation to compute the fracture pressure according to the tensile strength $T_{0}$ is

$$
P_{w c}=D-p-T_{0} .
$$

The final fracture pressure is then the smallest one computed from the equations in Table 4.2 and Equation (4-7).

After both breakout and fracture pressures are computed, (4-8) can be used to relate the pressures to their respective mud weights, 


$$
\rho_{m u d}=\frac{P_{w}}{g \times T V D},
$$

where $T V D$ stands for true vertical depth. Alternatively, other correlations can be used to consider the pressure drops and compute the equivalent circulating density.

A similar procedure is repeated in [21] for the Mogi-Coulomb criterion, but it will not be presented here. As mentioned, the Mogi-Coulomb criterion considers the influence of the mean principal stress and is less conservative if compared to the MC criterion. Both criteria used in [21] lead to a similar trend on the mud weight stability window, although the Mogi-Coulomb criterion yields a slightly larger window.

\section{2}

\section{Numerical Solution}

Several numerical procedures were proposed to solve a more general wellbore stability analysis or to deal with specific challenges in particular projects. Even though the analytical solution is still relevant for wellbore projects, the conditions required for the solution to be directly applicable (vertical well, no form of coupling, use of MC or the Mogi-Coulomb criterion, linear elastic conditions) may be too restrictive. Recent works tested different methodologies, including the use of Transversely Isotropic constitutive equations to create wellbore models [2] or the addition of uncertainties related to the data available for the analysis, either by considering the spatial variability of the material properties [57, 58], employing Reliability Assessment Methods [59] or Quantitative Risk Analysis [6].

In this work, we adopt the same strategy from $[57,58,60,42]$ to determine wellbore failure. Instead of assuming failure when the yield function reaches zero, the wellbore is allowed to yield to some extent. The function that describes the wellbore failure is

$$
F_{1}=A\left(P_{w}, t\right)-A^{*},
$$

where $A^{*}$ is the maximum allowable yielded area and $A\left(P_{w}, t\right)$ is the yielded area at time $t$ for the internal pressure $P_{w}$. Equation (4-9) is used to compute both breakout and fracture pressures, just like in Tables 4.1 and 4.2. Besides using Equation (4-9), the fracture pressure can also be determined with the original form of Equation (4-7),

$$
F_{2}=\sigma_{1}\left(P_{w}, t\right)-T_{0},
$$

where $\sigma_{1}\left(P_{w}, t\right)$ is the highest effective principal stress. 
In order to use Equations (4-9) and (4-10) to compute the ideal mud pressure window, any type of model is required provided it can evaluate the yielded area and the maximum effective stress. Here, a plugin-based FE simulator, discussed in Chapter 5, will be used to compute both results and solve the equations to determine the required internal pressures. The simulator will also be capable of computing the ideal mud pressure for inclined wells, considering different yield criteria and the poromechanical coupling, but will remain limited to the hypothesis presented in chapters 2 and 3.

The main goal of this approach to compute the ideal mud pressure window isn't to decide if Equations (4-9) and (4-10) are the most suited ones to model wellbore failure, but to derive a general framework capable of evaluating the optimal mud pressure and weight for a given failure condition. Other functions could also be used to verify if the wellbore deformation is within acceptable ranges, such as the breakout width, the diameter enlargement/reduction or sand production.

The final step to compute the optimal mud pressure window is to minimize and to maximize the mud pressure such that $F_{1} \leq 0$ and $F_{2} \leq 0$ and that the pressure remains inside the initial mud window based on the pore pressure, fracture pressure and lithostatic stress. Instead of performing an optimization analysis, the required mud pressures for each limit according to Equations (4-9) and (4-10) are computed by solving a root finding problem in the form of $F_{1}=0$ and $F_{2}=0$, since it consists of a one-dimensional problem, following $[42,57,58,60]$. The mud window will then be a combination of the roots of $F_{1}$ and $F_{2}$ and the initial mud window. A brief revision on the methods implemented in the simulator to solve this class of problems is presented next.

\subsection{1}

\section{NR and Accelerated NR}

The first method presented here is the Newton-Raphson method, already used in Chapter 3 to solve the nonlinear problem, together with a modification to increase its convergence rate. The general root-finding problem is defined as [52]:

$$
\text { given: } f: \mathcal{I}=(a, b) \subseteq \mathbb{R} \rightarrow \mathbb{R} \text {, find } x^{*} \in \mathcal{I} \text { such that } f\left(x^{*}\right)=0
$$

where the interval $\mathcal{I}$ is a subset of the real domain, $f$ represents either the yielded area or the maximum effective stress functions and $x^{*}$ is the root of $f$. The method computes a sequence of solutions $x_{k}$ using the iteration function 


$$
x_{k+1}=\phi\left(x_{k}\right)=x_{k}-\frac{f\left(x_{k}\right)}{f^{\prime}\left(x_{k}\right)}=x_{k}-\Delta
$$

to evaluate the $k+1$-th term. Since Equations (4-9) and (4-10) are evaluated as results of a numerical simulation and do not have an explicit form for the general problem, $f^{\prime}\left(x_{k}\right)$ is computed using a first order frontal finite difference scheme.

The NR method is one of the most efficient methods used to solve the root finding problem. The method presents a convergence of order 2 , at the expense of an increased cost per iteration to compute the derivative. However, it still has some drawbacks when used to solve the wellbore problem:

- It requires a initial guess in order to start the solution and converge to the right solution. This can be avoided by using the input data for the wellbore model (pore pressure, litostathic stress, etc) to estimate an initial guess;

- The value of Equation (4-9) depends on the current state of the material. If the wellbore enters the elastic domain during the solution of the problem, it may not be possible to compute the derivative at that point. Therefore, when using (4-9), initial guesses which are likely to be outside the mud pressure stability window are favored;

- Equation (4-9) itself is not continuous due to the spatial discretization of the model. The mesh around the wellbore should be as refined as possible to ensure that the value of $A\left(P_{w}, t\right)$ is close to $A^{*}$ and that the derivative does not evaluate to 0 for a small perturbation in the internal pressure;

Recently, a variant of the traditional NR method was proposed with a higher rate of convergence. The accelerated NR uses a numerical approximation of the second derivative of $f$ to compute a better prediction of $x_{k+1}$ [61]. The new iteration function now is

$$
x_{k+1}=\phi\left(x_{k}\right)=x_{k}-\frac{\Delta}{F}
$$

where

$$
F=\frac{1}{2}+\sqrt{\max \left(0, \frac{1}{4}-A_{2}^{e s t} \Delta\right)}
$$

and

$$
A_{2}^{e s t}=\frac{3 \frac{f\left(x_{k-1}\right)-f\left(x_{k}\right)}{x_{k-1}-x_{k}}-f^{\prime}\left(x_{k-1}\right)-2 f^{\prime}\left(x_{k}\right)}{\left(x_{k-1}-x_{k}\right) f^{\prime}\left(x_{k}\right)} .
$$

$A_{2}^{\text {est }}$ represents an estimate of the actual second derivative of $f$ and serves as a correction term for the derivative. If $x_{k}$ is close to the root, $A_{2}^{\text {est }} \Delta$ is close to 
zero and (4-13) tends to (4-12). On the other hand, if $A_{2}^{\text {est }} \Delta>0.25, F$ remains limited to 0.5 .

Even though the accelerated NR still has the disadvantages presented before, it has an increased convergence order of $\sqrt{3}+1$ [61]. Therefore, this method is expected to have at most the same number of function evaluations as the traditional NR, while only requiring that $x_{k-1}$ and $f\left(x_{k-1}\right)$ are saved and Equations (4-14) and (4-15) are evaluated. Additionally, seeing that (4-15) requires two pairs of point and function values to estimate the second derivative, the first step will always use the regular NR method, although different initialization strategies could be employed [61].

\subsection{2}

\section{False Position}

The second method discussed here is the False Position (FP) method, also known as Regula Falsi method. Unlike the NR method, the FP method requires a closed interval $\left[x_{a}, x_{b}\right]$ such that

$$
f\left(x_{a}\right) f\left(x_{b}\right)<0 .
$$

Therefore, the interval has at least 1 root of $f$. Again, the initial data of the model is used to define the input parameters for the FP method. The initial interval is defined based on the available pore pressure and lithostatic stress. Thus, if the initial interval indicates that the breakout pressure is outside the interval for instance, then the lower limit for the mud pressure would be the current pore pressure.

The method uses the following iteration equation to search for the roots inside the interval [52]

$$
x_{k+1}=\phi\left(x_{k}, x_{k^{\prime}}\right)=x_{k}-\frac{x_{k}-x_{k^{\prime}}}{f\left(x_{k}\right)-f\left(x_{k^{\prime}}\right)} f\left(x_{k}\right),
$$

where $x_{k}$ is the current point and $x_{k^{\prime}}$ is the last point such that Equation (4-16) is true for $f\left(x_{k}\right)$ and $f\left(x_{k^{\prime}}\right)$. After (4-17) is solved, $x_{k^{\prime}}$ becomes

$$
x_{k^{\prime}}= \begin{cases}x_{k} & \text { if } f\left(x_{k}\right) f\left(x_{k+1}\right)<0 \\ x_{k^{\prime}} & \text { otherwise }\end{cases}
$$

This procedure ensures that, throughout the solution, the interval will always have one root of the problem. The FP method also doesn't use the derivatives of the function, avoiding some of the NR problems related to derivatives at the cost of an order of convergence of 1 .

Several modifications to the traditional FP method are discussed in the literature. Here, the method is coupled with the Aitken's acceleration 
technique, also known as Aitken's $\delta^{2}$ method $[52,62]$. It is a general procedure initially proposed to convert a convergent sequence $x_{k}$ into a more rapidly convergent sequence. The technique is to compute the next step of the convergent sequence with

$$
x_{a i t}=\frac{x_{k} \phi\left(\phi\left(x_{k}\right)\right)-\phi\left(x_{k}\right)^{2}}{\phi\left(\phi\left(x_{k}\right)\right)-2 \phi\left(x_{k}\right)+x_{k}} .
$$

Note that, in the previous equation, $\phi\left(x_{k}\right)=x_{k+1}$ and $\phi\left(\phi\left(x_{k}\right)\right)=x_{k+2}$, which means that the FP method performs 2 regular iterations before using (4-19).

The main idea behind Equation (4-19) is to predict the next term of the sequence by extrapolating the error of each term, defined as

$$
e_{k}=\phi\left(x_{k}\right)-x_{k} .
$$

Since Equation (4-20) is 0 for $x^{*}$, the method tries to predict the next step by drawing a line between $\left(x_{k}, e_{k}\right)$ and $\left(x_{k+1}, e x_{k+1}\right)$. The result is Equation (4-19), where $x_{a i t}$ is the prediction from the extrapolation. For this reason, eq. (4-19) is also referred as Aitken's extrapolation formula.

Although the Aitken acceleration technique may seem simple, it yields good performance improvements. When it is used in conjunction with a linearly convergent method (e.g. the FP method), the Aitken method converges quadratically to the solution. However, when it is paired together with the FP method, the Aitken result should be used with care as it is not guaranteed it will remain inside the current interval.

Other modifications to the traditional FP method are reviewed in [63] and [64]. 


\section{Plugin based Simulator}

\section{1}

\section{Tectos and Ghem}

Tectos is a pre and post-processing software developed by the Geological Modeling (MGEO) group at Tecgraf/PUC-Rio. The program has tools to create bidimensional models by manually defining the limits of the section or by importing the geometry data from other programs. Once the user determines the geometry, the next step is to add different attributes and attach them to each face, edge and vertex of the model to represent the physical loads and conditions that the user wishes to simulate, as depicted in Figure 5.1. After the user informs all the inputs to the software, Tectos writes a neutral file ${ }^{1}$, which can be processed by the simulation software. Once the simulation ends, Tectos is also capable of reading simulation results and helping the user to visualize them, query results at specific points, create functions using the current results that generate new results, and export the result data as a table to be used by other software, among other functionalities.

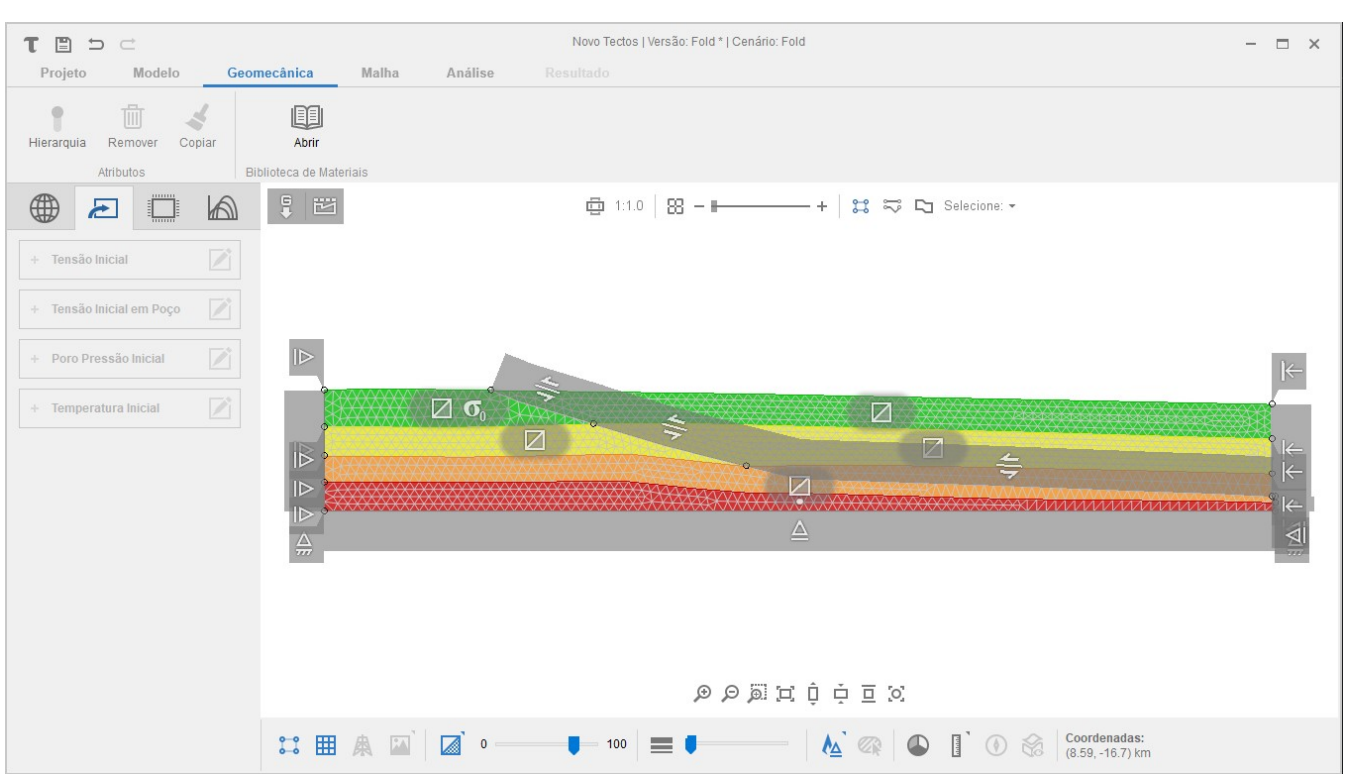

Figure 5.1: Tectos geomechanical attribute tab

${ }^{1}$ https://web.tecgraf .puc-rio.br/neutralfile/ 
The analysis itself, on the other hand, is handled by Ghem. It is a numerical simulation tool that uses the FEM to solve a variety of geomechanical problems, including viscoelastoplastic, thermo-fluid mechanical and incompressible analyses. It also can run parallel simulations using a shared-memory approach using the available threads on a CPU.

Both Tectos and Ghem were used in different geomechanical studies, e.g. rift extension modeling [65, 66], stress implication in karst formations [67, 68], salt tectonics $[69,70]$ and numerical simulation of field studies in different Brazilian basins $[71,72,73]$. Here, Tectos will provide the necessary input data for the simulations and will be used to visualize and export the results. All simulations will be performed using the new plugin simulator and Ghem will be used to assist in some validation examples.

\section{2}

\section{TopS Data Structure and TopSim Framework}

In this section, we present the tools used to create the simulator: the TopS data structure and the TopSim framework, both developed by the Reservoir and Visualization group at Tecgraf/PUC-Rio. They work together to provide the necessary resources to develop a complete and modularized simulator.

The TopS library is responsible for handling all topological information related to the model. It provides a complete, yet compact, data structure by explicitly representing nodes and elements while implicitly representing all other topological entities [74]. The library also provides the usual two- and tridimensional linear and quadratic elements used in FE analysis, but only T3 and Q4 are considered here. Besides topological data, TopS also creates and manages attributes associated with all topological entities, for instance model, element and node attributes. These attributes are used to represent all the necessary quantities related to a physical simulation, such as displacements, loads and pore pressures, and will be accessible to all plugins in the simulator. All related APIs to create and manipulate entities and attributes can be found in [74] and [75].

Meanwhile, the TopSim framework provides all the necessary tools to create and load all the desired plugins. The kernel of the framework is composed of the Plugin Manager. It is responsible for loading and querying plugins for the application, based on an input file. Unlike the previous one, where the neutral file format is used, this second input file is termed configuration file and is written using the Lua ${ }^{2}$ format, illustrated in Figure 5.2. It is essentially a set of tables describing the connectivity between plugins, their names and

\footnotetext{
${ }^{2}$ https://www.lua.org/
} 


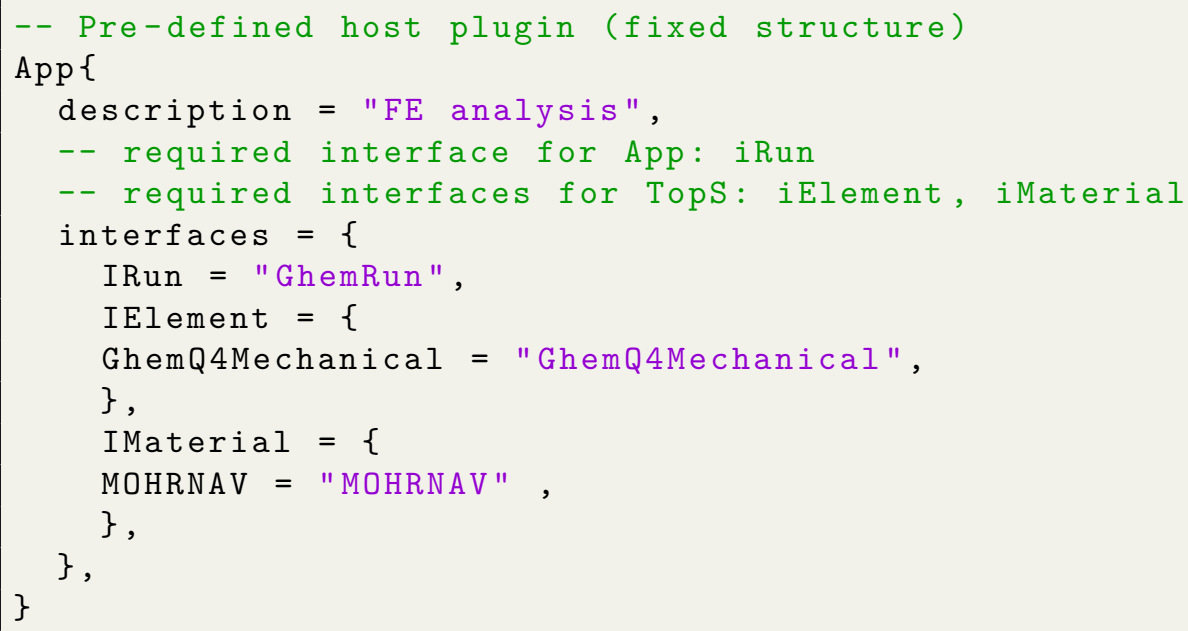

Figure 5.2: Example of Lua configuration file with the required plugins.

interfaces. At the start of the execution, the Plugin Manager reads and loads all the specified plugins. Once the file is processed, all plugins and the application itself can now use the manager to query other plugins and the simulation can start [75].

TopSim also requires a single model representation to run a simulation. It uses the TopS library to create such model and shares it with all loaded plugins. With this strategy, all plugins are capable of sharing information among themselves by creating and modifying model, element and nodal attributes, and of accessing all topological entities from the model with the TopS API. As a consequence, two different plugins do not need to be connected in order to share information nor the information needs to be defined in the API of a plugin, increasing the flexibility to develop and exchange new plugins in a larger scheme.

The third requirement to use the TopSim framework is the obligatory plugins in the configuration file. Every configuration file starts with a predefined host plugin, named "App" in Figure 5.2. It uses three other plugins: one plugin to provide the service IRun, which is the first user-defined plugin called in all simulations, one or more element plugins and an optional material plugin. Both element and material plugins will be available to all other plugins in the scheme through Plugin Manager. If one plugin requires any other plugin, it must be specified in the configuration file.

A few concepts need an introduction before discussing particular part of a plugin scheme in the next section. First, each plugin should perform a specific service for the application or other plugins. A service interface, or interface, specifies the API for each service. It is recommended to keep all 
arguments to a minimum in the API since TopS is responsible for sharing most of the information between plugins. Each plugin specifies the service execution with the implementation of the interface functions. They can also have plugin interfaces if they require other plugins to do a task for them [75]. For instance, consider the Newton Raphson plugin, detailed later. During its execution, it will call a function from the service interface ConvergenceCriterion to check if the solution converges. The actual metric used to check convergence will depend on the plugin specified in the configuration file. Once the plugin computes the value, it is stored in an attribute in the model, where the NR plugin checks to compare to a given tolerance.

\section{3}

\section{Plugin Scheme}

Figure 5.3 presents a general plugin scheme used to run a mechanical analysis and will be used as an example to show different aspects of the plugin simulator. Element and material plugins are omitted here and will be discussed at the end of this section. The scheme in Figure 5.3 is divided into four major groups: one responsible for controlling the simulation flow, one for I/O communication, one regarding the analysis itself and a last one to solve the linear system.

\subsection{1 \\ Main, Driver and Algorithm}

The main plugin provides the service IRun required by the host plugin to start a simulation. All plugins that implement this service also receive the command arguments that were informed when the application started. Here, the main plugin processes two arguments: the names of the neutral file with the input data, mandatory for every analysis, and an optional argument to specify the name of the output file. Both are stored as model attributes and will be used by the I/O plugins to open and create the required files. After the plugin finishes processing the arguments, it proceeds to call the reader plugin to create all necessary attributes, elements and nodes, and then a driver plugin, detailed next.

Driver plugins are responsible for controlling the evolution of a simulation. Here, two driver plugins are used: a LoadStepDriver and a TimeStepDriver, for transient analyses. The LoadStepDriver, presented in Figure 5.3, implements the regular load step strategy, commonly used in mechanical analyses, solving the current analysis with successive load increments until the full load is applied to the model. It reads the required number of load steps from 
a model attribute and creates a new load step factor attribute to control the load. During the simulation, it loops through the number of load steps, updates the load factor for each step, and calls for a Algorithm plugin to solve the current analysis. The plugin also requires up to two different Writer plugins: one obligatory plugin to write model results at user defined print-steps during the analysis and one optional plugin to write algorithm results, such as total number of iterations and error, for every loadstep.

An Algorithm plugin should specify the necessary steps to solve the current analysis. In the example of Figure 5.3, the simulator uses a NR algorithm plugin to solve a mechanical problem. The plugin itself doesn't make any computations; it only calls the Analysis service to compute the residual vector, the Jacobian matrix and the solution increment at the current step. The operations are performed by the other plugins, updating the necessary attributes at each stage of the NR algorithm. To keep the $N R$ plugin as general as possible, it also needs a ConvergenceCriterion plugin to compute the error at the current iteration. In the scheme represented in Figure 5.3, the ResIncrProd plugin computes the error and stores the value in an attribute. The $N R$ plugin reads the value and compares it with an user-defined tolerance to check the convergence. Another feature of the $N R$ plugin is the optional requirement of a Writer plugin to update the output information at every iteration.

Besides the $N R$ plugin, other implemented Algorithm plugins are the StaggeredProcedure and the FalsePosition. The former is used in coupled analyses, as illustrated in Figure 5.7 discussed later, while the latter implements the FP method to solve one-dimensional problems (e.g. computation of the ideal internal pressure at a given depth). The FalsePosition plugin will also use an Analysis, a ConvergenceCriterion and a Writer plugin, in a similar fashion to the $N R$ plugin.

\subsection{2}

\section{Reader and Writer}

Reader and writer plugins will be responsible for the communication between the simulator and other pre- and post-processing tools. They should be freely exchangeable to allow for the integration with multiple software, but should always use the same attributes to ensure compatibility with the other plugins.

Only one reader plugin is used here, NfReader, which specializes in reading the neutral file with all the tags used by Tectos. The model, empty at first, is populated as the plugin reads the topological data. Then, the plugin reads the remaining information of the file, such as nodal supports, prescribed 
pore pressure and initial stresses, creating separate attributes for each one. Once it reaches the final tag of the file, all data has been added to the model, and the simulation is ready to start.

During the simulation, when a Driver plugin reaches a user-specified load or time step or at the end of an Algorithm plugin iteration, it may request a writer plugin to save the current state of the model to an output file. The $N f r B i n$ plugin then retrieves specific nodal attributes, e.g. nodal displacement and pore pressure, and begins to save the current results. It also loops through the elements and uses the IElement plugin from the Plugin Manager to compute specific simulation results at the Gauss points and writes them in the output file. Finally, it writes all data in a binary file format using a third-party library developed at Tecgraf/PUC-Rio to export results to Tectos. This highlights another feature of a modular simulator: it doesn't oblige the whole simulator to depend on specific libraries; only the plugins that use an external library will require it.

\subsection{3}

\section{Analysis, DoFManager and Boundary Condition}

The Analysis, DoFManager and BoundaryCondition services are related to the problem itself. A combination of all three plugins will determine which kind of analysis will be solved. In this example, the StaticFEM, DisplacementDoF and MechanicalBdryCnd plugins indicate that the scheme will perform a static (or quasi-static) FE analysis to compute the displacements in a body.

The StaticFEM plugin is a general plugin to perform static FE analysis. As required in the Analysis service interface, the plugin must define the methods to compute the residual vector, the Jacobian matrix, to solve the system and to update the attributes with the solution of the linear system. In the example illustrated in Figure 5.3, the $N R$ plugin asks the StaticFEM plugin to compute the residual vector. The StaticFEM first calls for an BoundaryCond service to process analysis-specifc boundary loads and then loop through the elements, using the IElement service to compute the element-wise residual vector. For each element contribution, the StaticFEM assembles the element vector in a global residual vector according to the degree of freedom (DOF) numbering. As the $N R$ algorithm proceeds, the StaticFEM again loops through the elements and uses the IElement service to compute the element-wise Jacobian matrix, but this time also uses a SparseMatrix service to assist in the matrix assembly. A third important task of the StaticFEM plugin is to solve the resulting linear system. Since each FE analysis may benefit of different 
linear solvers, the plugin uses a LinearSolver service to compute the system solution. With the solution at hand, it calls for a DOFManager service to update analysis specific attributes and then uses the IElement service interface one more time to update element information. With this implementation, the StaticFEM plugin becomes a versatile plugin for different FE analyses, only defining the general routines for FE simulation.

Both DoFManager and BoundaryCond services are separated from the StaticFEM plugin to handle the different attributes for each kind of analysis. In this example, the DisplacementDoF plugin provides the DoFManager service. It creates and handles analysis specific attributes (e.g. displacements), initializes attributes used by different plugins, such as the global load vector and generates a DOF numbering, also stored as an attribute. The MechanicalBdryCnd provides the BoundaryCond service and follows a similar philosophy. It handles analysis-specif boundary conditions, such as nodal loads and supports, and stores this information in the previous global load vector when requested.

\subsection{4 \\ LinSolver and SparseMatrix}

The LinSolver and SparseMatrix services are required to solve the matrix system obtained when using the FEM. Here, we use the PCG [52] to solve all linear systems from Chapter 3 since the Jacobian matrices from Equations (3-8a) and (3-8b) are symmetric and positive definite. The plugin MgeoSolverWrapper works in a similar manner to the NfrBin plugin: it uses the MgeoSolver library, a third-party library developed by the MGEO group, with a parallel version of the solver. The plugin retrieves all necessary attributes and the sparse matrix and passes both as arguments to the library.

The SparseMatrix is a service designed to help the simulator manipulate the Jacobian matrix in a memory efficient sparse format. Instead of storing all the matrix entries, a sparse representation omits some or all null elements. In Figure 5.3, the CSRMatrix plugin uses the Compressed Sparse Row (CSR) format to store the matrix, saving only the nonzero entries, their column and the index of the non zero array for each row [75], since the solver library requires a CSR representation. All other plugins use a SparseMatrix plugin if they need to manipulate the matrix and do not demand a specific representation. Other used formats are the Compressed Sparse Column (CSC) and the Skyline $[12,75]$, but these will not be used here. 


\subsection{5}

\section{Other Plugins}

A few other plugins are present in the scheme to provide minor services for others. One example is the ConvergenceCriterion plugins, usually requested by Algorithm plugins. Each plugin implements its metric to check convergence based on specific attributes. Then, the error is stored in an attribute in the model to be used by other plugins. An example is the the ResIncrProd plugin, from Figure 5.3. It retrieves the current residual vector and the last linear system solution to compute the convergence error as

$$
e_{i}=\frac{\sum_{k=1}^{N}\left|\boldsymbol{R}_{k, i} \delta \boldsymbol{x}_{k, i}\right|}{\left\|\boldsymbol{R}_{0}\right\|}
$$

where $\left\|\boldsymbol{R}_{0}\right\|$ is the Euclidean norm of the initial residual vector and $e_{i}, \boldsymbol{R}_{k, i}$ and $\boldsymbol{x}_{k, i}$ are respectively the convergence error, the k-th entry of the residual vector and the $\mathrm{k}$-th term of the solution increment at the i-th iteration of the NR algorithm. 


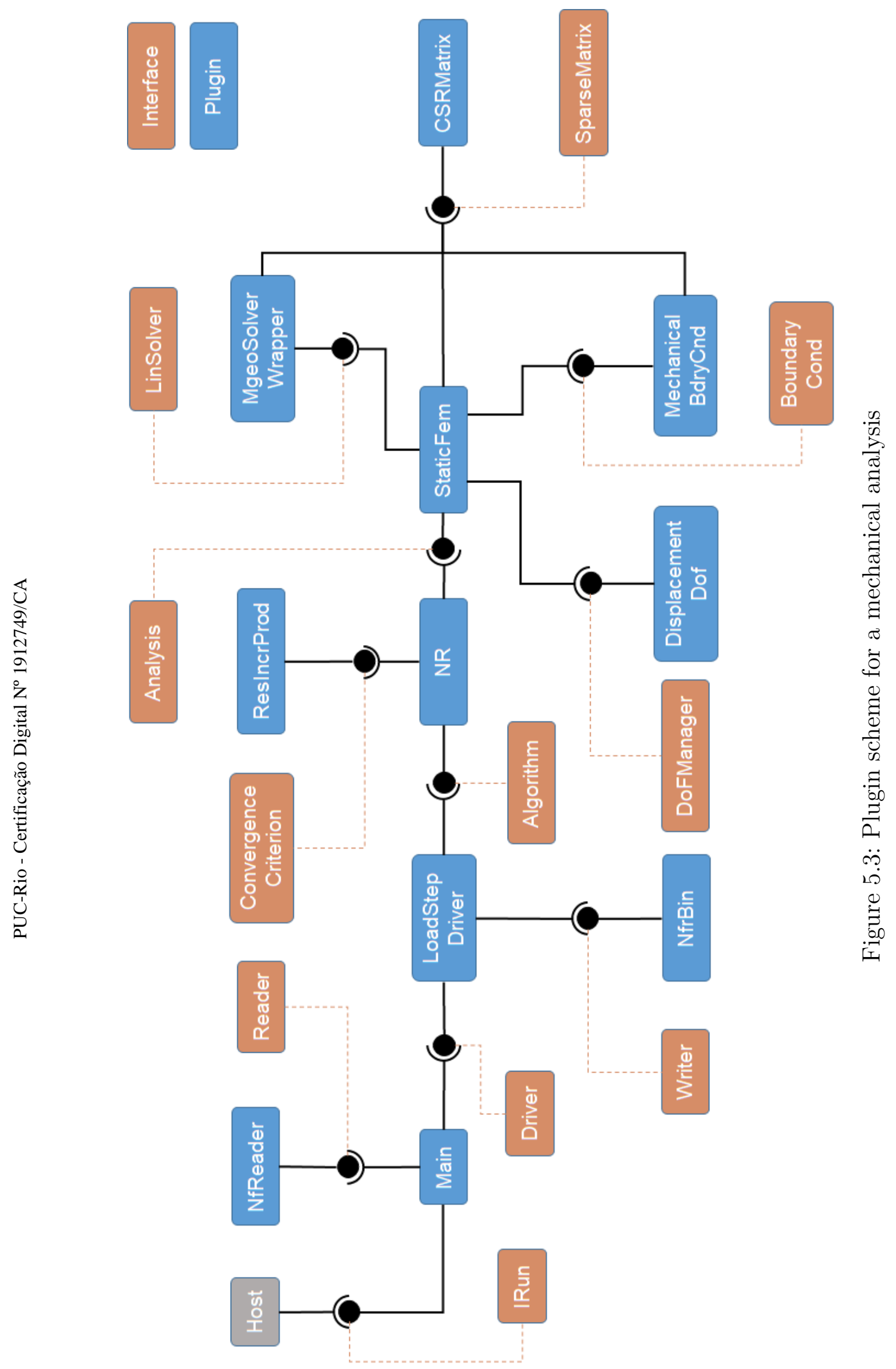




\subsection{6 IElement}

Unlike the previous plugins, IElement plugins work slightly differently in the scheme. The host plugin requires an IElement plugin to start a simulation, depicted in Figure 5.2, and it is not connected to any other plugins in the scheme. Once all plugins are loaded from the configuration file, all element plugins are added to the Plugin Manager and can be accessed by any other plugin using the manager's API in Figure 5.4 at any point of a simulation.

The element plugin itself - or rather, its scheme - is represented in Figure 5.5. The plugin MechanicalFEAnalysis is the element plugin used in this example and it is responsible for computing the residual vector of an element and the element Jacobian for a mechanical problem, in addition to the body and surfaces forces. It requires two other services: the ShapeFunc service and the WorkConjugate. The first one calculates the element shape functions and its derivatives at the element Gauss points, in this case for a Q4 element. The second service computes the element-wise Jacobian matrix and internal forces contributions to the residual vector, since both are related to the chosen compatibility and constitutive equations.

The Engineering plugin provides the WorkConjugate service, indicating that the current analysis assumes infinitesimal strains and uses the Cauchy stress tensor. The plugin then requests other services such as Compatibility, Constitutive and PlaneStrain, to compute the compatibility matrix and the tangent operator and to update the current stresses and strains. In Figure 5.5, the plugin ContinuumPln returns the compatibility matrix for continuum

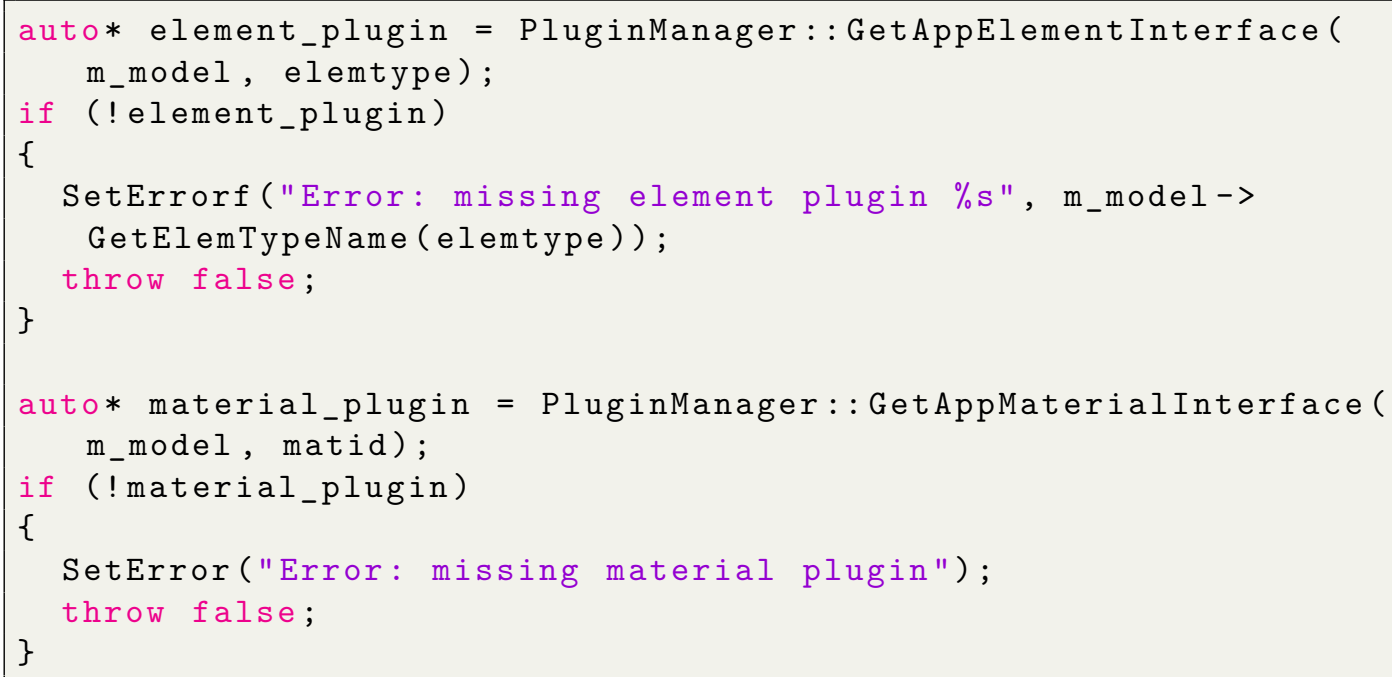

Figure 5.4: Plugin Manager function calls to request elements and materials 
elements under plane stress or strain states, whereas the PlnHooke plugin provides the tangent operator and the PlaneStrain plugin assists in the computations under plane strain conditions. The engineering and PlnHooke plugins also use the IMaterial service during their execution, but the necessary plugins are requested directly from the Plugin Manager and are omitted in Figure 5.5.

The main goal behind the element plugin scheme presented in Figure 5.5 is to achieve a flexible design that can be re-utilized in different analyses. A similar scheme, where a T3 plugin replaces the $Q 4$ plugin, solves a FE analysis using T3 elements, or the Engineering plugin may be replaced by a new plugin which considers the large displacement effects in the simulation and demands the non linear compatibility matrix from the plugin ContinuumPln. Other analyses also benefit from the plugins presented here, such as the single-phase fluid flow analysis discussed in Chapters 2 and 3, where the compatibility matrix may be used to account for the contributions of the displacement field.

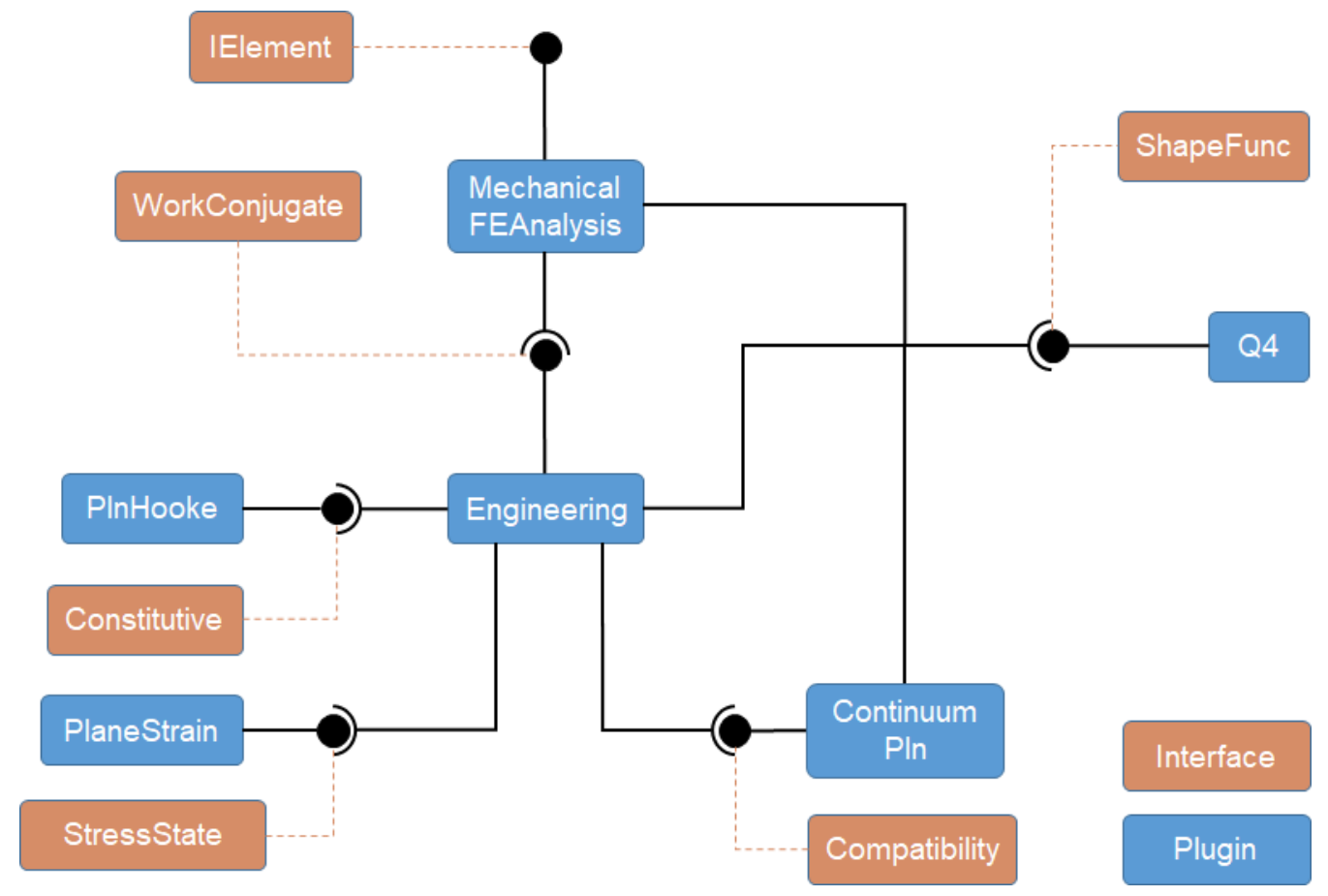

Figure 5.5: Element plugin scheme in a mechanical analysis

\subsection{7}

IMaterial

Material plugins work similarly to element plugins: they are also obligatory in the host plugin and can be accessed by any other plugin during the simulation through the Plugin Manager. Figure 5.6 shows a simple design for 
an elastoplastic analysis with a von Mises material. The plugin MechanicalMat uses both an ElasticBehavior service to model the elastic behavior as well as a ReturnMapping and a YieldCriterion service to model the plastic behavior. The IsoHooke plugin computes the elastic tangent operator, while the CPA plugin updates the stresses for a given strain increment and, in this situation, computes the continuum elastoplastic tangent operator. The vonMises plugin computes the VM yield function and its derivatives, when requested by the $C P A$ plugin.

In this scheme, the CPA was implemented in a separate plugin from the yield criterion since the algorithm leads to a simple implementation where it only needs the yield function, yield function derivatives and plastic potential derivatives. Another option would be to merge both plugins and create a return mapping plugin attached to a specific yield criterion [14]. This merged implementation allows for modifications on the return algorithm to benefit from certain characteristics of each criterion, a trade-off between a more general solution and a more specific and optimized one. A secondary advantage of designating the Return Mapping service as a part of the material scheme is that a single simulation may naturally handle multiple materials, each one with a different Return Mapping algorithm for the local plasticity problem, using only the TopSim API.

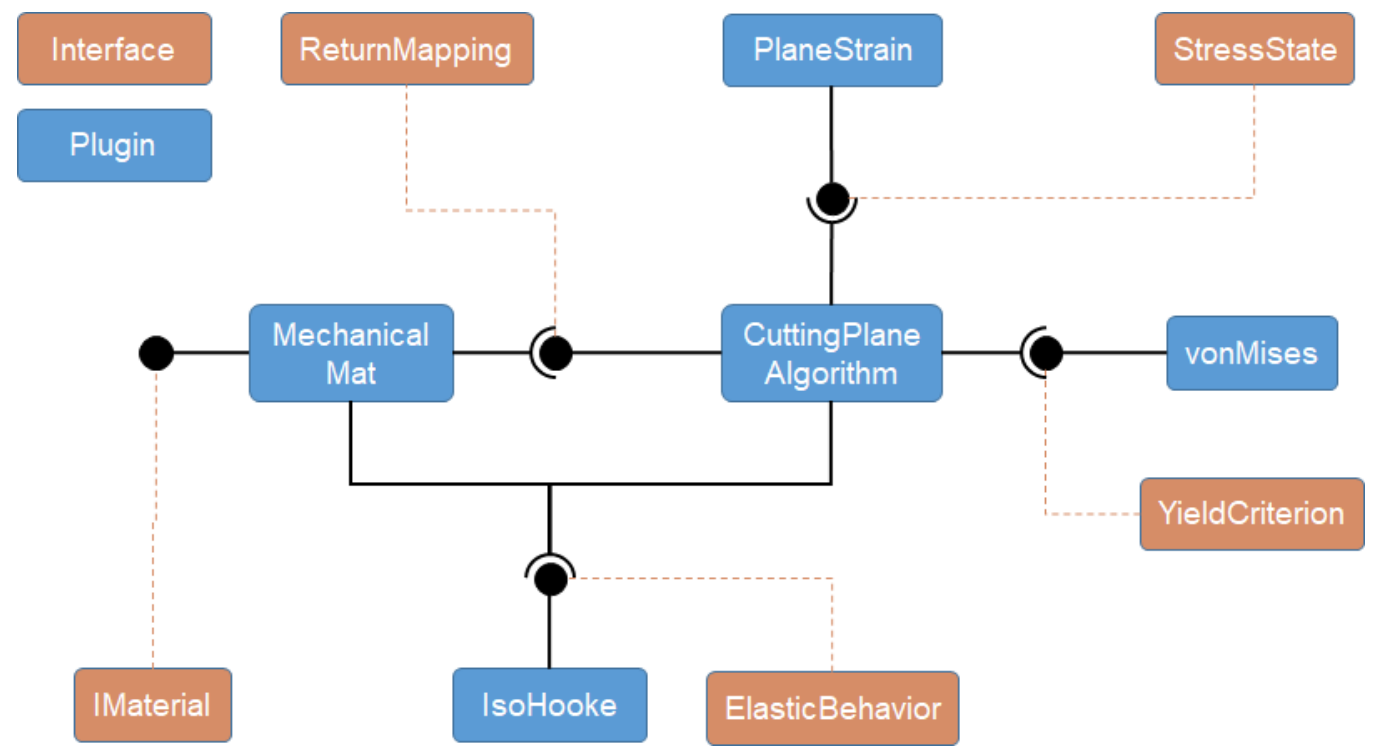

Figure 5.6: Material scheme for a VM material 


\subsection{8 Well Stability Scheme}

The plugin scheme presented so far provides the necessary tools to perform a FE simulation and serves as the basis to run different analyses. For instance, to solve the well stability problem, a slightly altered scheme - one that is capable of running coupled analyses - is used alongside other plugins as shown in Figure 5.7. The new one uses a different main plugin, specific for well stability analysis, that handles multiple output files and requires an Algorithm service. This example uses the same $N R$ plugin, but this time the optional Writer plugin is present. During the stability analysis, the Algorithm plugin calls for the writing process at each iteration and uses a different ConvergenceCriterion and Analysis plugins. While the plugin L2Relative only computes the relative norm, the RestrictionAnalysis plugin solves equation (4-9) or (4-10) using a Simulator interface since its solution requires a numerical simulation. Any third-party software is feasible here as long as there is a plugin that communicates with the simulator. In Figure 5.7, a PluginSimulator scheme indicates that it will connect itself with a plugin scheme similar to the previous one to run the simulation.

Figures 5.8 and 5.9 summarize the modifications required to run a coupled analysis. The simulation demands a TimeStepDriver to control the solution process. During each time step, it calls the Staggered plugin, responsible for the staggered procedure. It solves the mechanical and the fluid flow analysis until the displacement and pore pressure fields converge. The Mechanical Scheme in Figure 5.8 corresponds to a similar scheme to the one presented in Figure 5.3, starting at the NR plugin. The Fluid Flow Scheme, on the other hand, uses a SPPorePressureDOF and a SPPorePressureBdryCnd plugins to provide the DOFManager and BoundaryCond services, respectively. With the solution of the current time step at hand, the TimeStepDriver calls the TimeStepHeuristic service to compute the next time step and the simulation proceeds until it reaches the total simulation time. The Driver plugin in Figure 5.8 also uses the binary file writer plugin to save simulation results at user-specified time steps.

As for the element scheme in Figure 5.9, it must provide the required services for both mechanical and fluid flown analyses. For this reason, a StaggeredFEAnalysis plugin retrieves a model attribute from TopS to determine which FEAnalysis plugin should be used to compute element-wise vectors and matrices. 


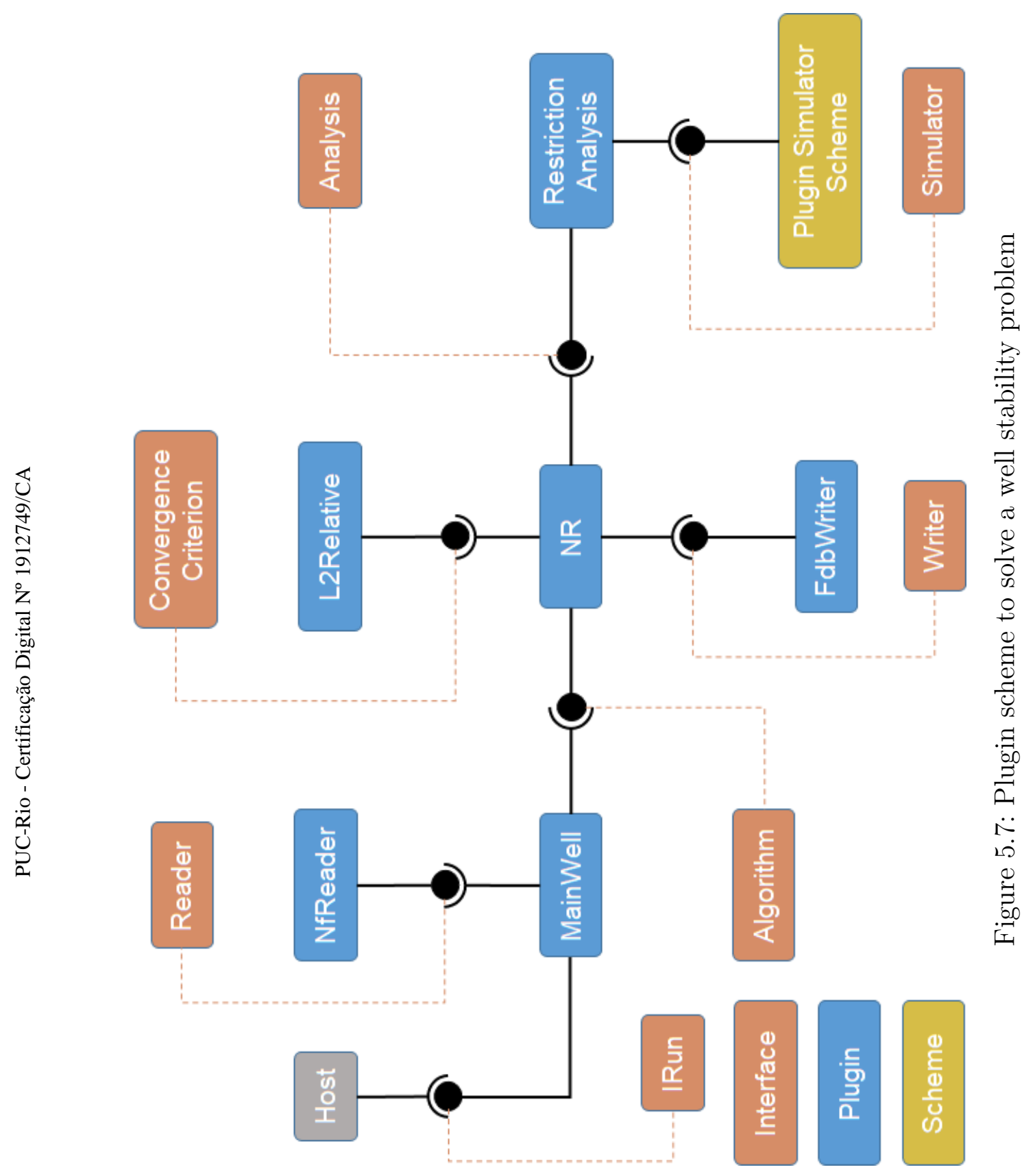




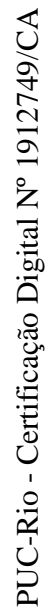

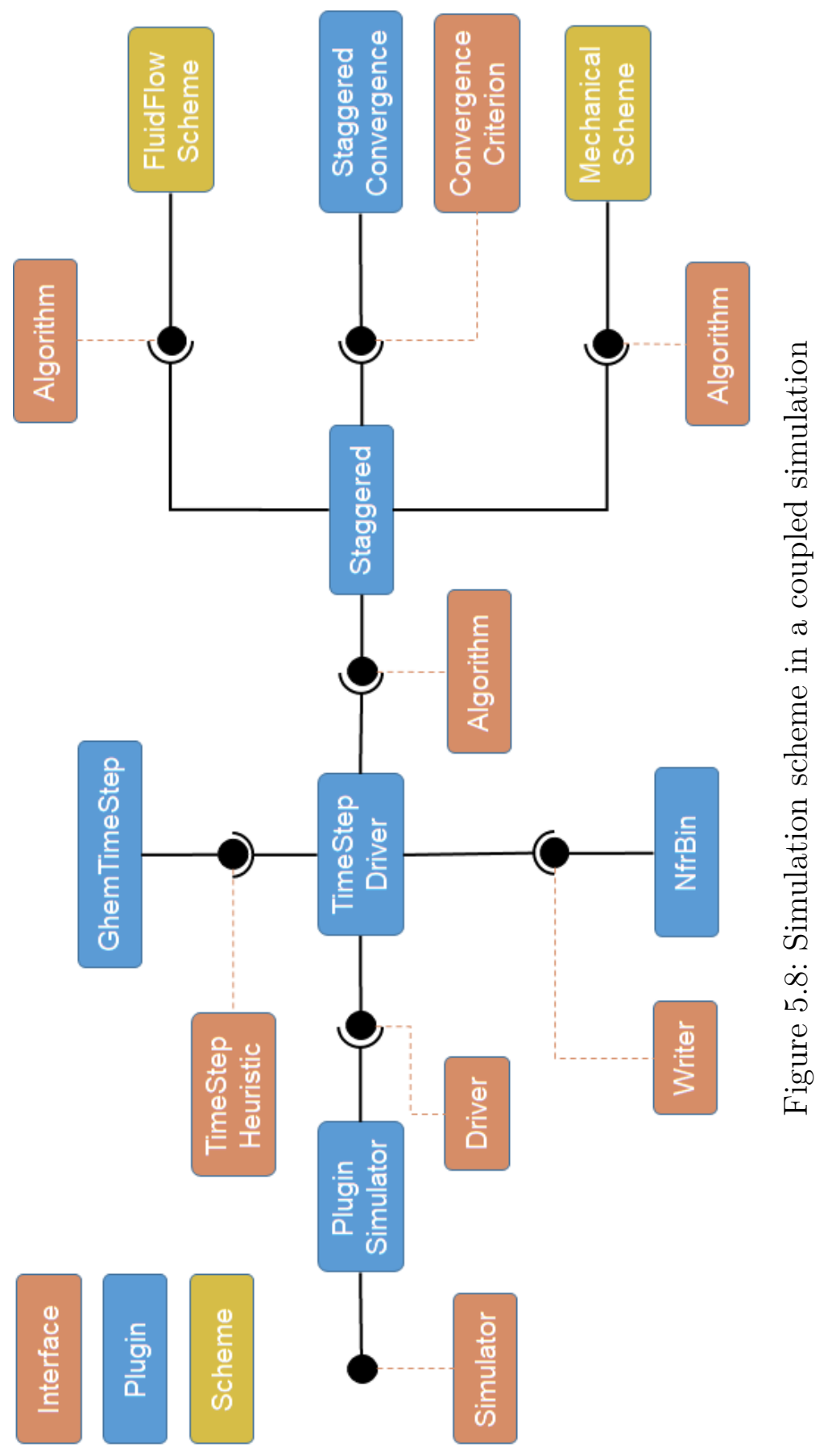




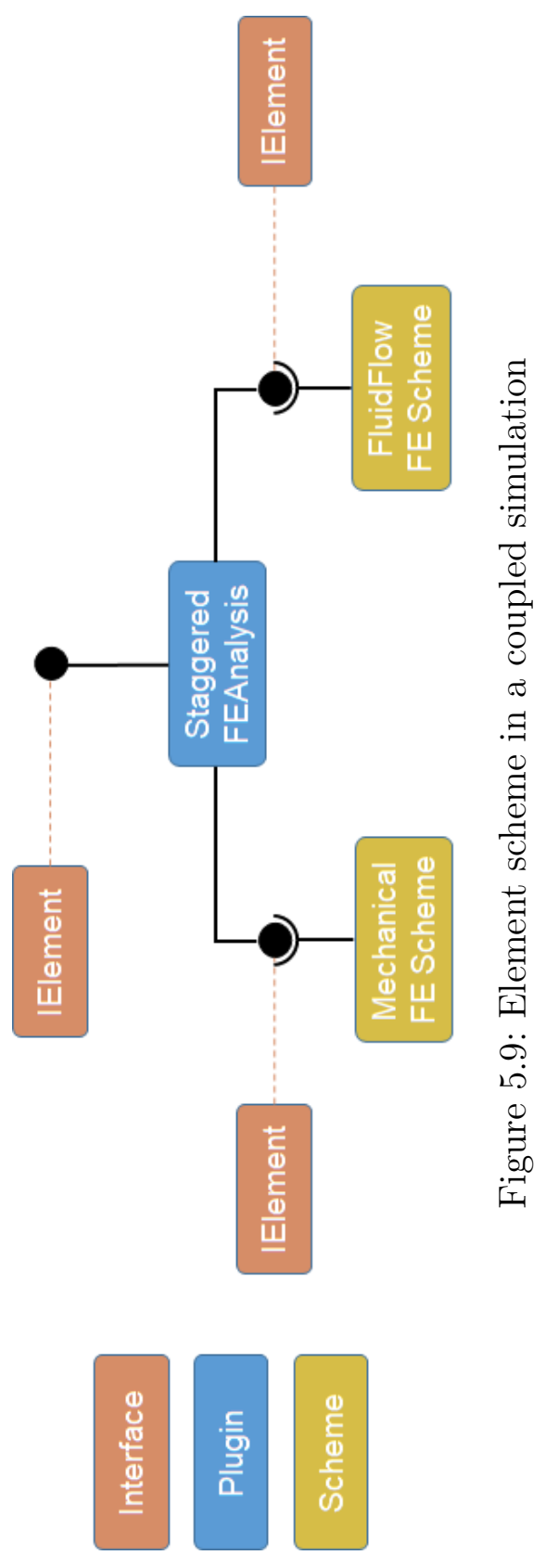




\section{Validation}

This chapter contains a set of simple problems to test and validate the implementations and the simulator presented so far. Tectos was used to generate all models and meshes and it was also responsible for reading the result files from the simulator and exporting the results to a numeric data file. The data in the exported file is then compared to the analytical solution of each problem using a Matlab script.

\section{1}

\section{Elastoplastic Cylinder with von Mises Material}

The first example is a long elastoplastic thick-walled cylinder subjected to internal pressure [14]. The model is a cross-section of the cylinder, shown in Figure 6.1, assumed to be in plane strain conditions. In Figure 6.1, $a, b$ and $c$ are the inner, outer and plastic front radius, respectively, and $P$ is the internal pressure. The VM criterion is used to model the plastic behavior of the material assuming an elastic-perfectly plastic behavior.

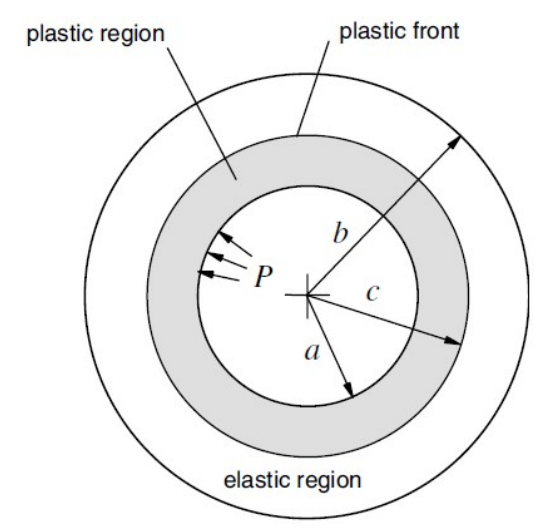

Figure 6.1: Cross-section of the elastoplastic cylinder [14]

If $P$ is too low, the material will remain within the elastic range and the analytical solution is given by Lamé's equations. Once the internal pressure surpasses a certain threshold, the stress distributions in the plastic area, $a \leq r \leq c$, are given by [76]:

$$
\sigma_{r r}=Y\left[-\frac{1}{2}-\ln \left(\frac{c}{r}\right)+\frac{c^{2}}{2 b^{2}}\right]
$$


and

$$
\sigma_{\theta \theta}=Y\left[\frac{1}{2}-\ln \left(\frac{c}{r}\right)+\frac{c^{2}}{2 b^{2}}\right]
$$

where $Y=2 * \sigma_{Y} / \sqrt{3}$ and $r$ is the radial coordinate. In the elastic area, $c \leq r \leq b$, the stresses are given by:

$$
\sigma_{r r}=-\frac{Y c^{2}}{2 b^{2}}\left(\frac{b^{2}}{r^{2}}-1\right)
$$

and

$$
\sigma_{r r}=\frac{Y c^{2}}{2 b^{2}}\left(\frac{b^{2}}{r^{2}}+1\right)
$$

The plastic front radius can be calculated by solving Equation (6-5) for a given internal pressure.

$$
\frac{P}{Y}=\ln \left(\frac{c}{a}\right)+\frac{1}{2}\left(1-\frac{c^{2}}{b^{2}}\right) .
$$

The parameters used for this model are represented in Table 6.1. Two values for internal pressure were used: $75 \mathrm{MPa}$, in the elastic range, and 180 $\mathrm{MPa}$, in the plastic range. The plastic front radius in the Table 6.1 corresponds to the internal pressure in the plastic range. Figure 6.2 shows the geometry of the model used in this example. Only a quarter of the cross-section is represented, with a 50x70 Q4 mesh.

\begin{tabular}{ccc}
\hline Parameter & Unit & Value \\
\hline Inner Radius (a) & $\mathrm{mm}$ & 100 \\
\hline Outer Radius (b) & $\mathrm{mm}$ & 200 \\
\hline Plastic Front $(\mathrm{c})$ & $\mathrm{mm}$ & 159.29 \\
\hline Young Modulus $(\mathrm{E})$ & $\mathrm{GPa}$ & 210 \\
\hline Poison $(\nu)$ & - & 0.3 \\
\hline Yield Strength $\left(\sigma_{Y}\right)$ & $\mathrm{MPa}$ & 240 \\
\hline
\end{tabular}

Table 6.1: Parameters for the cylinder model [14]

As seen in Figures 6.3 and 6.4, all numerical results matches the analytical solutions presented in the elastic and elastoplastic ranges. 


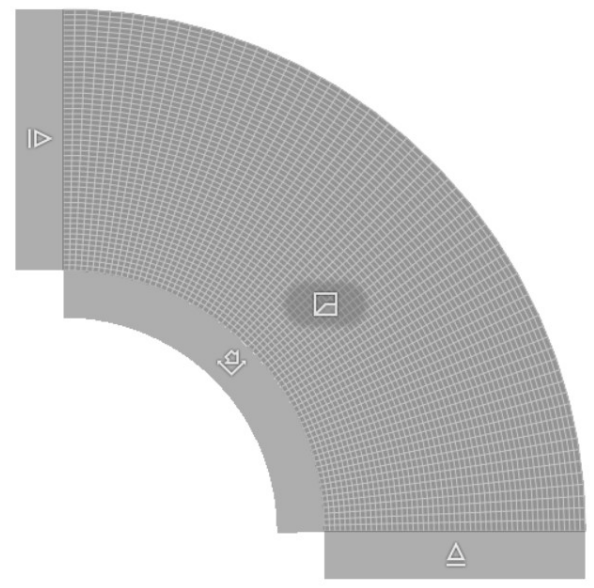

Figure 6.2: Quarter cylinder

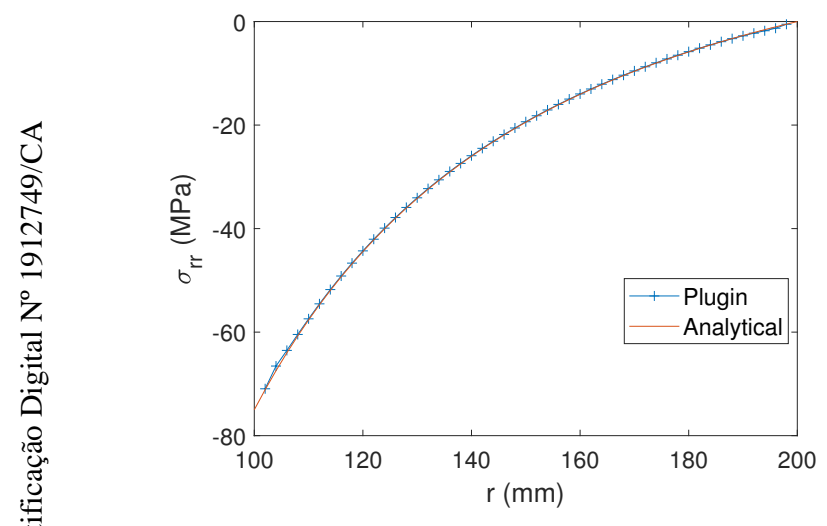

(a) Radial stress

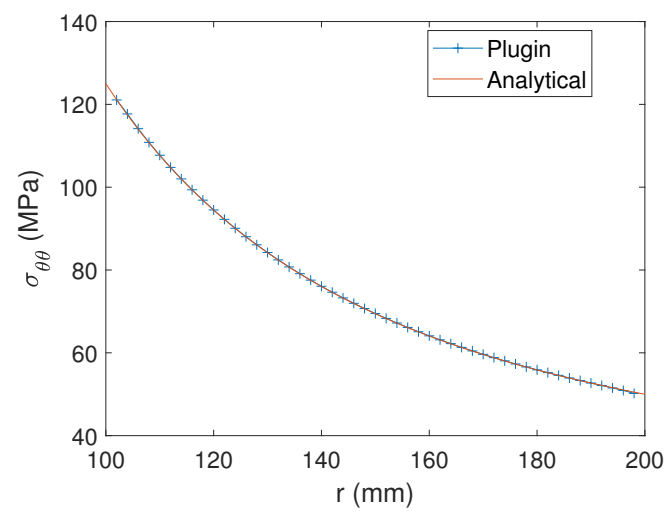

(b) Hoop stress

Figure 6.3: Stresses vs radial coordinate, $75 \mathrm{MPa}$

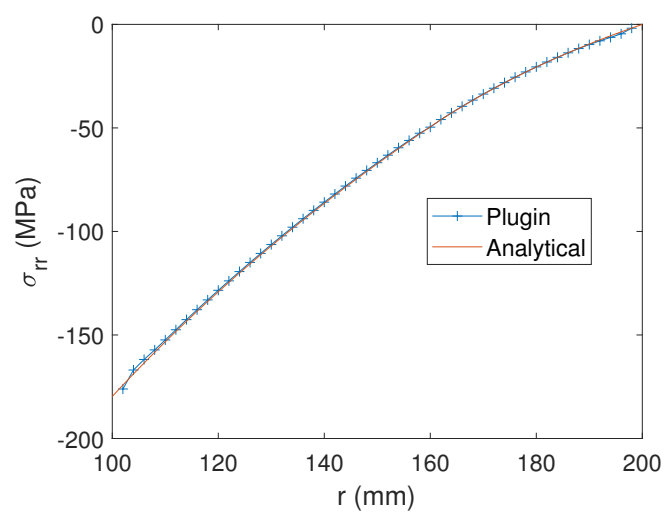

(a) Radial stress

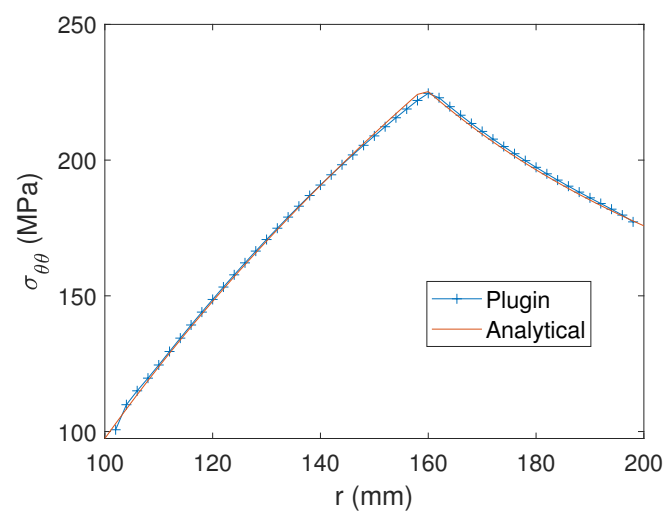

(b) Hoop stress

Figure 6.4: Stresses vs radial coordinate, $180 \mathrm{MPa}$ 


\section{2}

\section{Circular hole in a Infinite Mohr Coulomb Material}

The next validation model is similar to the previous one. This time, the elastic-perfectly plastic behavior is modeled by the Mohr-Coulomb criterion and the model, depicted in Figure 6.5, can be seen as a cylindrical hole in a soil. Unlike the von Mises model, this time a bi-axial isotropic stress state is applied to all four faces faces and the displacement is restricted in the outer edges of Figure 6.5. It is assumed that the situation represented by this model is still under plane strain conditions [77].

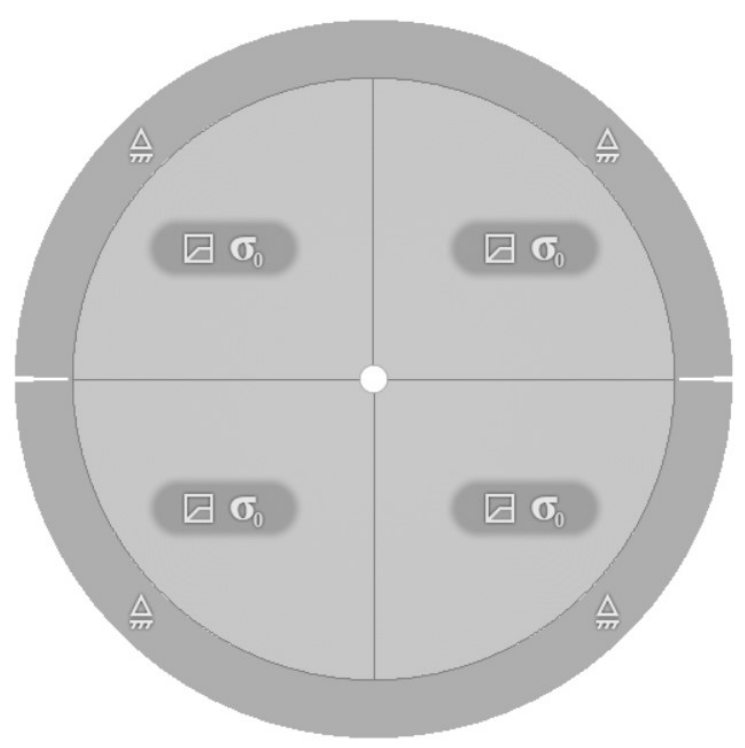

Figure 6.5: Cylindrical hole in a Mohr Coulomb material

The solution of this problem is given in [78]:

$$
\sigma_{r r}=P-\left(P-\frac{1}{K_{p}+1}\left(2 P-K_{c}\right)\right)\left(\frac{R_{0}}{r}\right)^{2},
$$

for the radial stresses, and

$$
\sigma_{\theta \theta}=P+\left(P-\frac{1}{K_{p}+1}\left(2 P-K_{c}\right)\right)\left(\frac{R_{0}}{r}\right)^{2},
$$

for hoop stresses. In Equations (6-6) and (6-7), $P$ represents the stresses acting in both directions, $r$ is the radial coordinate and $R_{0}$ is given by:

$$
R_{0}=a\left(\frac{2}{K_{p}+1} \frac{P+\frac{K_{c}}{K_{p}-1}}{\frac{K_{c}}{K_{p}-1}}\right)^{\frac{1}{K_{p}-1}}
$$

where $a$ is the hole radius, and both $K_{p}$ and $K_{c}$ are given by Equations (6-9) and (6-10), respectively.

$$
K_{p}=\frac{1+\sin \phi}{1-\sin \phi}
$$




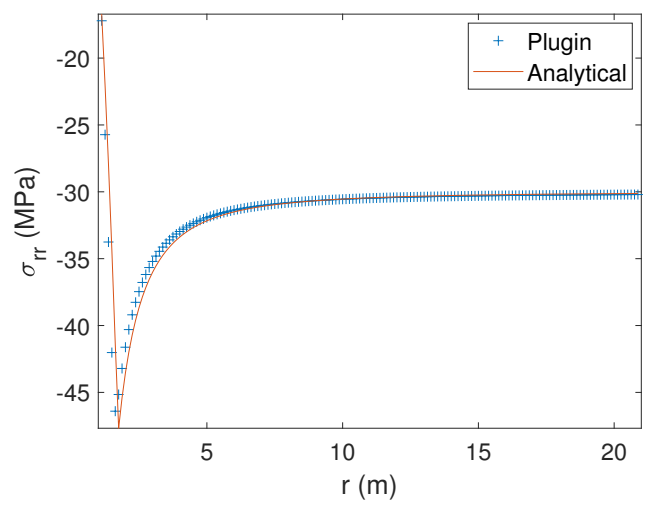

(a) Radial stress

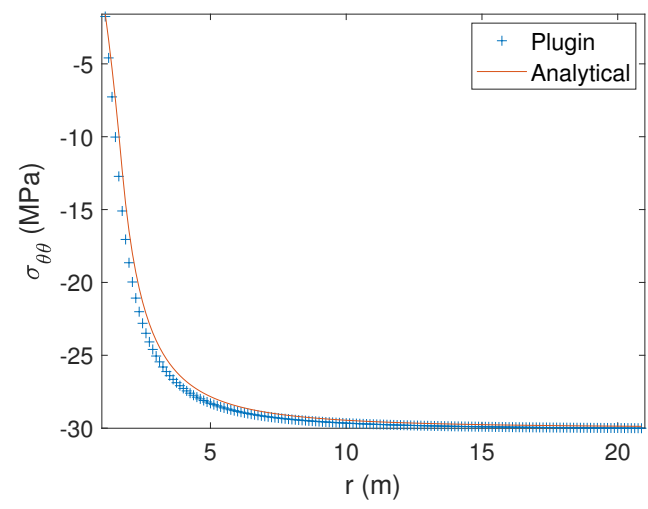

(b) Hoop stress

Figure 6.6: Stresses vs radial coordinate

$$
K_{c}=2 c \tan \left(\frac{\pi}{4}+\frac{\phi}{2}\right)
$$

Table 6.2 shows the material, geometry and load input parameters used in this model, where $\mathrm{P}$ is the value applied in $\sigma_{x x}$ and $\sigma_{y y}$. The mesh consists of a 160x80 Q4 mesh on each quadrant, totalizing 51200 elements.

\begin{tabular}{ccc}
\hline Parameter & Unit & Value \\
\hline Inner Radius $(\mathrm{a})$ & $\mathrm{m}$ & 1 \\
\hline Outer Radius $(\mathrm{b})$ & $\mathrm{m}$ & 21 \\
\hline Young Modulus $(\mathrm{E})$ & $\mathrm{GPa}$ & 10 \\
\hline Poison $(\nu)$ & - & 0.2 \\
\hline Cohesion $(c)$ & $\mathrm{MPa}$ & 3.45 \\
\hline Friction Angle $(\phi)$ & $\circ$ & 30 \\
\hline Pressure $(P)$ & $\mathrm{MPa}$ & 30 \\
\hline
\end{tabular}

Table 6.2: Parameters for the MC hole model [77]

Figure 6.6 compares the analytical results with the numerical ones. By directly applying the prescribed stresses directly on each surface, the material starts to yield at a non differentiable point of the yield surface and the entire model yields. However, the techniques described in [15] proved to be sufficient to ensure good numerical results.

\section{3}

\section{Oedometric Test}

The third validation test represents an oedometer test in a soil sample to verify the implementation for the MCC model. As mentioned in chapter 
3 , the Young modulus is assumed to change according to the hydrostatic compressive pressure acting on the medium. The model, represented in Figure 6.7, has an initial hydrostatic stress acting on the material and is subjected to a compressive load acting on the upper edge, while all other edges have their movement restricted. The mesh used in this model consists of a 50x20 Q4 elements. All the remaining parameters in this model are summarized in Table 6.3.

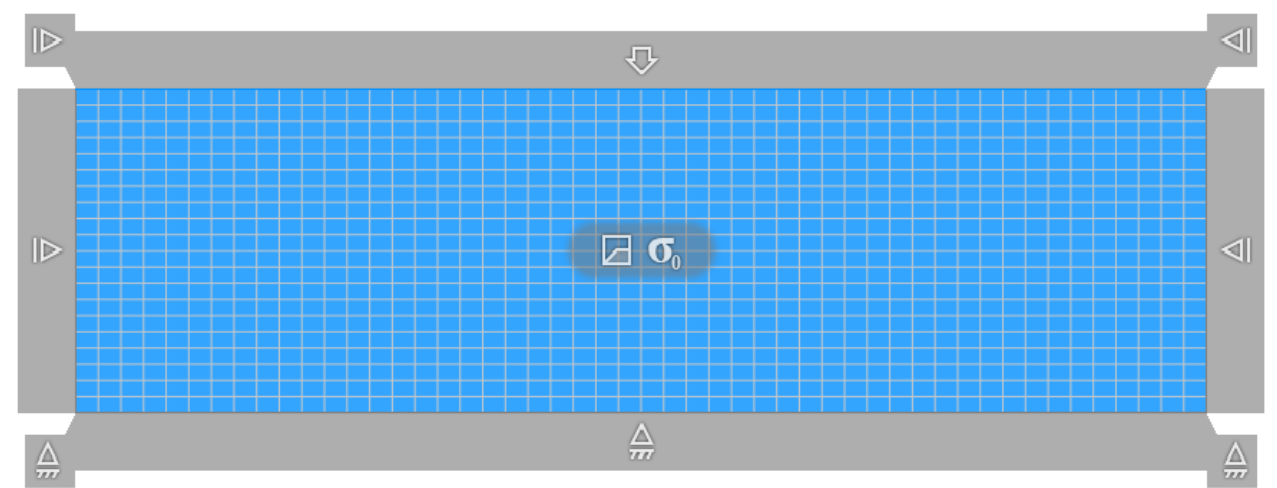

Figure 6.7: Oedometer test model

\begin{tabular}{ccc}
\hline Parameter & Unit & Value \\
\hline Dimensions $(\mathrm{LxH})$ & $\mathrm{mm}$ & $70 \times 20$ \\
\hline Hyd. Stress & $\mathrm{MPa}$ & -0.1 \\
\hline Load & $\mathrm{MPa}$ & -0.3 \\
\hline Poison $(\nu)$ & - & 0.3 \\
\hline \# Loadsteps & - & 50 \\
\hline$\beta$ & - & 1 \\
\hline Critical State Line Slope $(\mathrm{M})$ & - & 1.2 \\
\hline Hyd. Yield Stress $\left(P_{t}\right)$ & $\mathrm{MPa}$ & 0 \\
\hline Virgin Consolidation Line Slope $(\lambda)$ & - & 0.077 \\
\hline Swelling Line Slope $(\kappa)$ & - & 0.0066 \\
\hline Initial ellipsis radius $\left(a_{0}\right)$ & $\mathrm{MPa}$ & 0.1 \\
\hline Initial void ratio $\left(e_{0}\right)$ & - & 0.788 \\
\hline
\end{tabular}

Table 6.3: Parameters and loads for the MCC hole model [30]

The model was simulated using the plugin simulator and its results were compared to the results from Ghem. All results were taken in a point at the 
middle of the model. Figures 6.8 to 6.11 show the results for this simulation. It is possible to see that all the results from the plugin simulator match the ones from the current simulation, with a small difference in the final Young modulus.

Figure $6.10 \mathrm{~b}$ shows the behavior of the model is compatible to the one in Figure 2.5. The preconsolidated soil follows the swelling line until approximately the $20^{\text {th }}$ loadstep, when it starts to yield. From there on, the material returns to the virgin compression line and continues to yield until the end of the analysis. The slope of both sections of the line in Figure $6.10 \mathrm{~b}$ are approximately $-6.6918 \times 10^{-3}$ and $-7.808710^{-2}$, in agreement with the material parameters used for the simulation.

Figure 6.11 also shows the same characteristic. Assuming that the soil, in its virgin state, was loaded with an hydrostatic stress of $-0.2 \mathrm{MPa}$ and then unloaded until it reached -0.1 MPa, it would end up at the the point $(-0.1,0)$ in Figure 6.11. After, as the vertical load increases under plane strain conditions, the soil deforms elastically inside the yield surface and then plastically as the ellipsis radius (or preconsolidation pressure) increases until the end of the simulation.

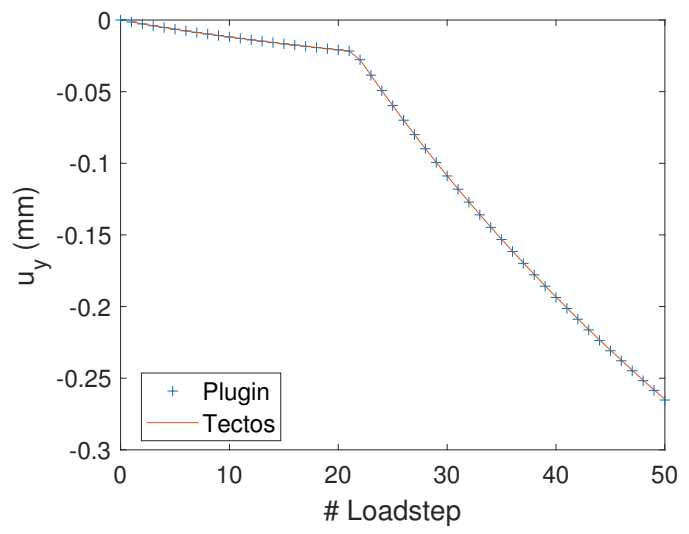

Figure 6.8: Vertical displacement vs \# Loadsteps 


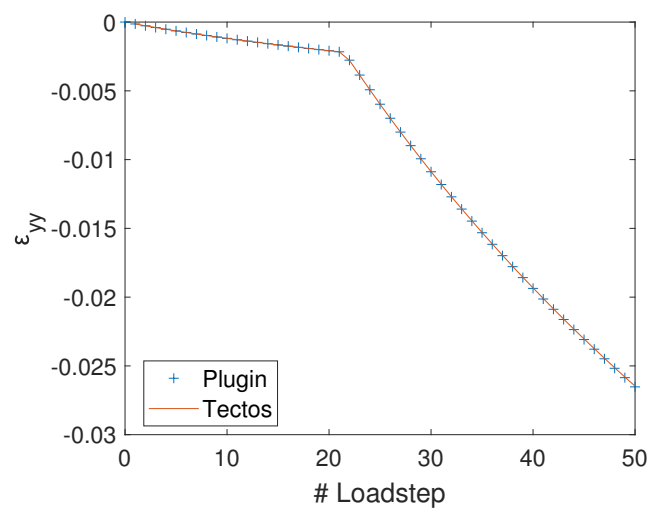

(a) Total vertical strain

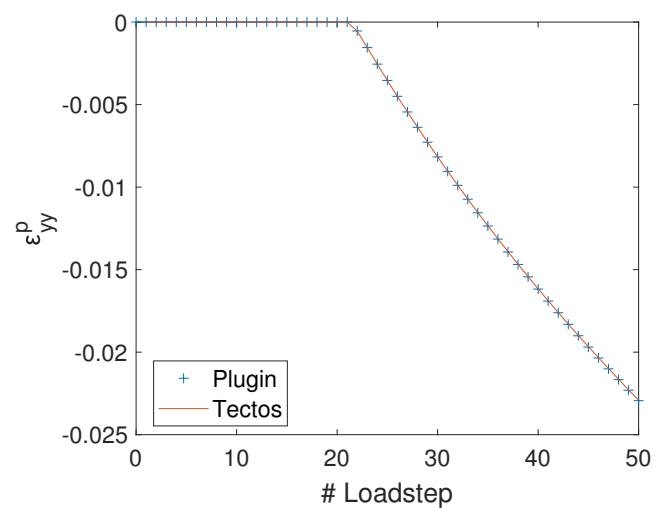

(b) Plastic vertical strain

Figure 6.9: Strains vs \# Loadsteps

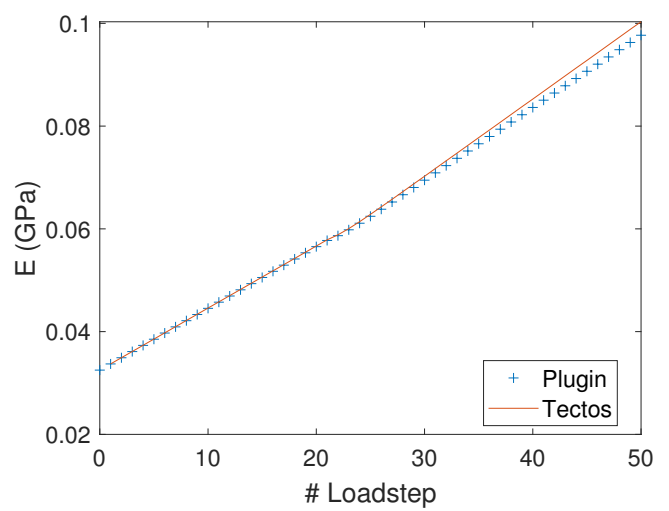

(a) Young Modulus

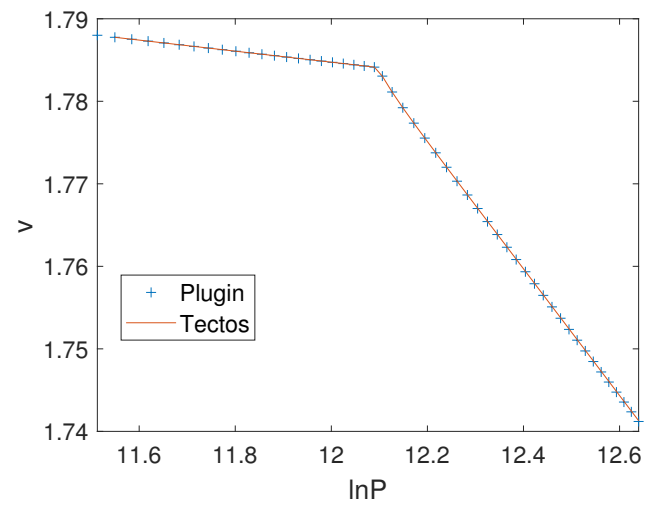

(b) $\ln \mathrm{P}$ vs specific volume

Figure 6.10: Variation of the Young modulus and relation lnP-specific volume of the throughout the simulation

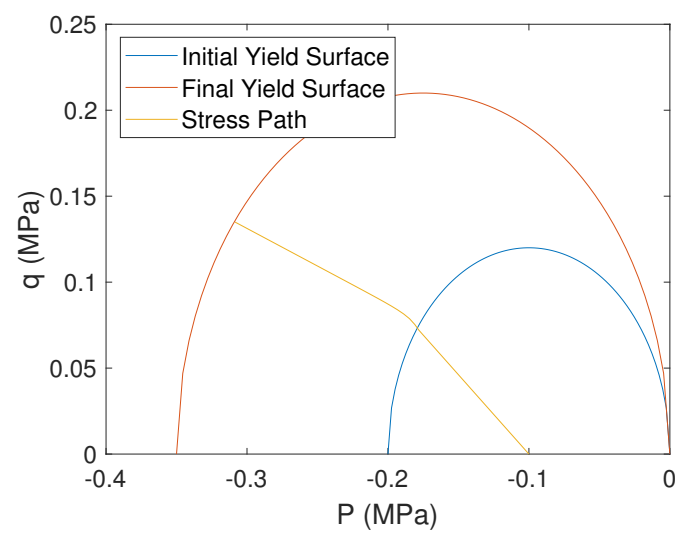

Figure 6.11: Stress path and yield surface in the P x q space 


\section{4}

\section{Poroelastic Column}

In order to validate the implementation used for the coupled problem, two more test cases were used. The first one represents a one dimensional consolidation problem of a saturated poroelastic column. Figure 6.12 shows the model used to simulate this problem, where a load $p *$ and a prescribed pore pressure are applied on the top edge of the model. All parameters used in this model are summarized in Table 6.4.

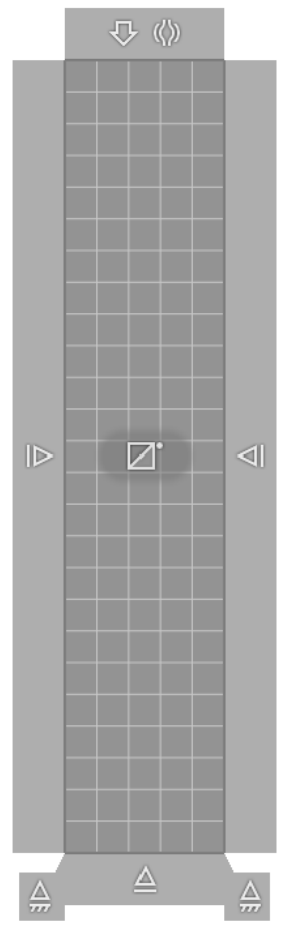

Figure 6.12: Poroelastic column model

\begin{tabular}{ccc}
\hline Parameter & Unit & Value \\
\hline Dimensions $(\mathrm{LxH})$ & $\mathrm{m}$ & $1.4 \times 7$ \\
\hline Load $\left(p^{*}\right)$ & $\mathrm{MPa}$ & -1 \\
\hline Prescribed pore pressure & $\mathrm{MPa}$ & 0 \\
\hline Initial pore pressure & $\mathrm{MPa}$ & 0 \\
\hline Young Modulus $(E)$ & $\mathrm{GPa}$ & 1.44 \\
\hline Poison $(\nu)$ & - & 0.2 \\
\hline Biot $(\alpha)$ & - & 0.7778 \\
\hline Permeability $(k)$ & $\mathrm{mD}$ & 190 \\
\hline Porosity $(n)$ & - & 0.19 \\
\hline Viscosity $(\mu)$ & $\mathrm{Pas}$ & 0.001 \\
\hline
\end{tabular}


Continuation of Table 6.4

\begin{tabular}{ccc}
\hline Parameter & Unit & Value \\
\hline Grain Compressibility $\left(K_{s}\right)$ & $\mathrm{GPa}$ & 36 \\
\hline Fluid Compressibility $\left(K_{w}\right)$ & $\mathrm{GPa}$ & 2.887 \\
\hline Simulation Time & $\mathrm{s}$ & 100 \\
\hline
\end{tabular}

Table 6.4: Parameters and loads for the poroelastic column model [42]

The analytical solution for this problem uses the following undrained properties [42]:

$$
\nu_{u}=\frac{3 K_{u}-2 G}{2\left(3 K_{u}+G\right)}
$$

and

$$
K_{u}=K\left(1+\frac{\alpha^{2} K_{f}}{(1-\alpha)(\alpha-n) K_{f}+n K}\right),
$$

where $\mathrm{K}$ is the bulk modulus of the soil matrix, $\mathrm{G}$ is the shear modulus and $K_{u}$ and $\nu_{u}$ are the undrained bulk modulus and Poison coefficient, respectively. The pore pressure field is then given by:

$$
p=\frac{\eta p^{*}}{G S}\left[1-F_{1}(\chi, \tau)\right]
$$

In equation $6-13$, the final pore pressure field is computed using $F_{1}$, an infinite series:

$$
F_{1}(\chi, \tau)=1-\sum_{k=1}^{\infty} \frac{2}{M(k)} \sin (M(k) \chi) e^{-4 \tau M(k)^{2}},
$$

where $\chi$ and $\tau$ are the adimensional depth and time,

$$
\chi=\frac{y}{H}
$$

and

$$
\tau=\frac{2 k G(1-\nu)\left(\nu_{u}-\nu\right)}{\alpha^{2}(1-2 \nu)^{2}\left(1-\nu_{u}\right)} \frac{t}{4 H},
$$

respectively, and

$$
M(k)=\frac{\pi(2 k+1)}{2} .
$$

$\eta$ and $S$ are

$$
\eta=\frac{\alpha(1-2 \nu)}{2(1-\nu)}
$$

and 


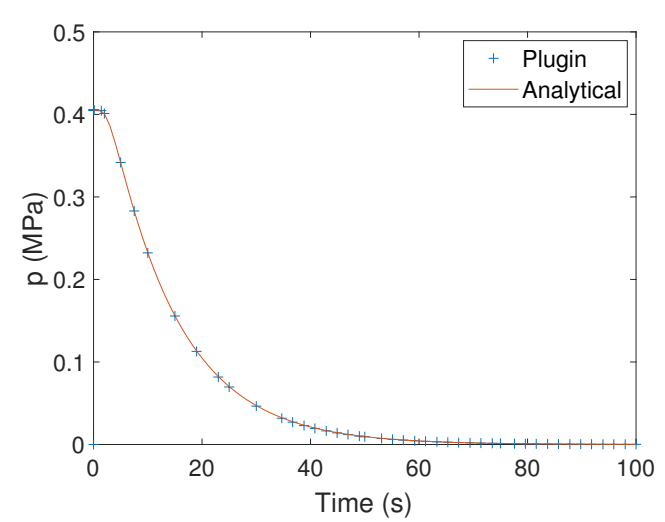

(a) Pore pressure at the bottom of the column during the simulation

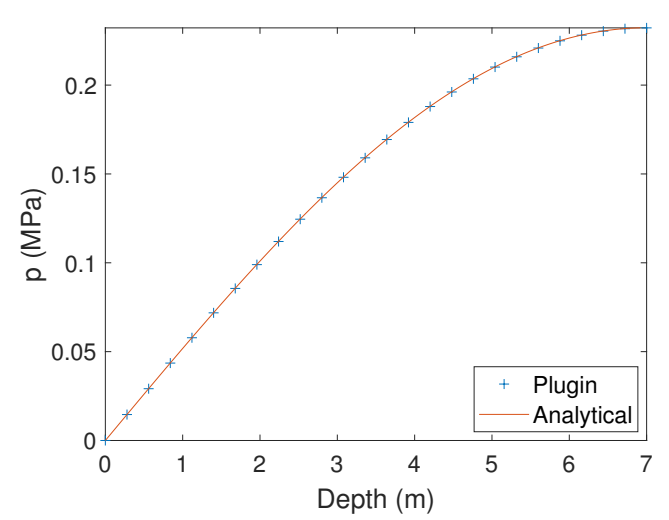

(b) Pore pressure along the middle of the column at $10 \mathrm{~s}$

Figure 6.13: Pore pressure distribution in the poroelastic column

$$
S=\frac{3 \eta\left(1-\nu_{u}\right)}{G B\left(1+\nu_{u}\right)}
$$

where B is the Skempton coefficient:

$$
B=\frac{\alpha K_{f}}{(\alpha-n(1-\alpha)) K_{f}+n K} .
$$

The problem was solved using the plugin simulator with the coupled scheme. Figures $6.13 \mathrm{a}$ and $6.13 \mathrm{~b}$ shows the pore pressure results computed via the simulator and Equation (6-13). The first image shows the variation of the pore pressure at a point located in the middle of the bottom of the column. There is a sudden increment due to the undrained response as the loads are instantly applied and then the pore pressure dissipates as the simulation advances. The second picture shows the distribution of the pore pressure field along a line through the middle of the column 10 seconds after the start of the simulation. Both numerical results matched the analytical ones.

\section{5}

\section{Poroelastic Wellbore Problem}

The final validation example is a simulation of the poroelastic response of a borehole. This model represents a simplified version of the actual drilling process. The material is assumed to be under a non hydrostatic stress state and to have a constant initial pore pressure field. Then, an "instantaneous" borehole is introduced in the middle of the model and all internal forces and pore pressure at the edges of the borehole are removed. It is assumed that this problem occurs at plane strain conditions, provided that the time needed to drill through a distance equal to five times the borehole radius is greater than a characteristic time $t_{c}$ in Equation (6-21) [79]. 


$$
t_{c}=\frac{a^{2} \mu(1-2 \nu)}{2 k G(1-\nu)}
$$

This problem was first solved by Detournay and Cheng in [79]. The authors solved this problem by sub dividing it in three loading modes (an axysimmetric load, a purely deviatoric load and a prescribed pore pressure at the borehole wall) and superposing the solution of the three modes. While the first loading mode is solved using the Lamé solution for thick cylinders, the other two modes are solved in the Laplace transform domain. The complete solution for all three modes in the Laplace domain as well as the solution in the time domain at small times can be found in [79]. Here, the numerical results are compared to the small time solution of the stresses and pore pressure fields.

Figure 6.14 represents the model used to solve this problem with the plugin simulator. It consists of a square model with a hole in the center and a Q4 mesh with 40x40 elements. All results were taken at the yellow line, with $21.037^{\circ}$ of inclination, after 0.1 seconds in order to ensure the small time condition. Table 6.5 presents all material parameters and loads used in this simulation.

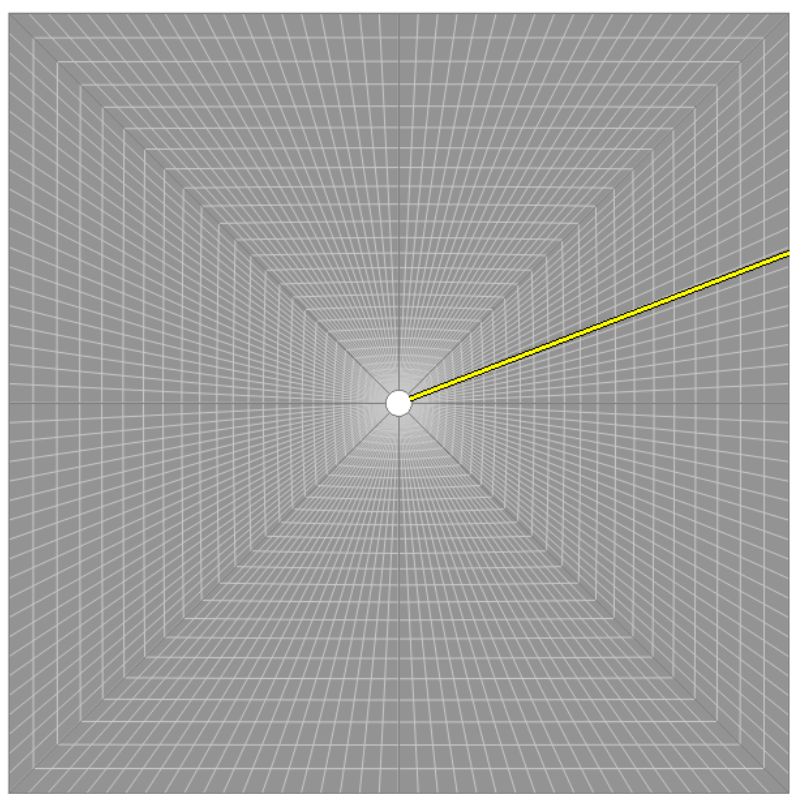

Figure 6.14: Wellbore model

\begin{tabular}{ccc}
\hline Parameter & Unit & Value \\
\hline Dimensions $(\mathrm{L})$ & $\mathrm{m}$ & 6 \\
\hline Wellbore Radius $(\mathrm{a})$ & $\mathrm{m}$ & 0.1 \\
\hline Initial Effective Horizontal Stress $\left(\sigma_{0, x x}^{\prime \prime}\right)$ & $\mathrm{MPa}$ & -30 \\
\hline Initial Effective Vertical Stress $\left(\sigma_{0, y y}^{\prime \prime}\right)$ & $\mathrm{MPa}$ & -50 \\
\hline
\end{tabular}


Continuation of Table 6.5

\begin{tabular}{ccc}
\hline Parameter & Unit & Value \\
\hline Initial pore pressure $\left(p_{0}\right)$ & $\mathrm{MPa}$ & 15 \\
\hline Young Modulus $(E)$ & $\mathrm{GPa}$ & 1.44 \\
\hline Poison $(\nu)$ & - & 0.2 \\
\hline Biot $(\alpha)$ & - & 0.7895 \\
\hline Permeability $(k)$ & $\mathrm{mD}$ & 1.9 \\
\hline Porosity $(\phi)$ & - & 0.19 \\
\hline Viscosity $(\mu)$ & $\mathrm{Pas}$ & 0.001 \\
\hline Grain Compressibility $\left(K_{s}\right)$ & $\mathrm{GPa}$ & 38 \\
\hline Fluid Compressibility $\left(K_{w}\right)$ & $\mathrm{GPa}$ & 2.884 \\
\hline Total Time & $\mathrm{s}$ & 10 \\
\hline
\end{tabular}

Table 6.5: Parameters and loads for the well model [42]

Figures 6.15 and 6.16 show the results for this simulation. The introduction of a borehole in the medium locally disturbs the stress and pore pressure fields. This disturbance is a result of the stress concentration effects from the borehole together with the pore pressure variation and persists until approximately 1 meter from the center of the borehole, where all results return to their respective far field values. As the simulation continues, the pore pressure dissipates even further into the model and its effects can be seen in greater distances. All figures indicate that the simulator is capable of reproducing the analytical results. 


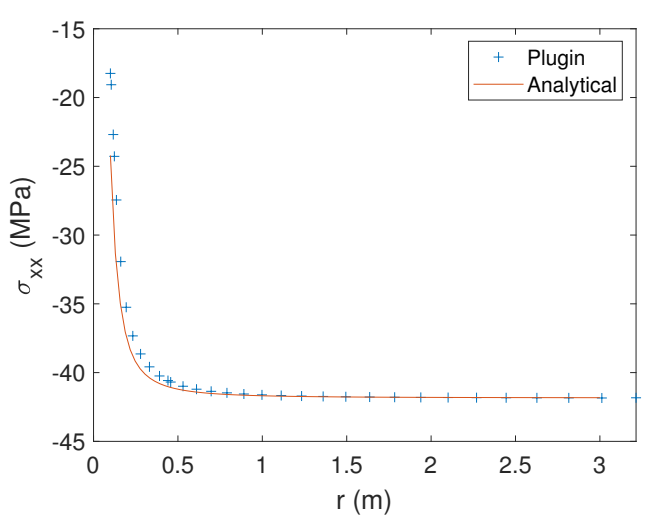

(a) Horizontal stresses

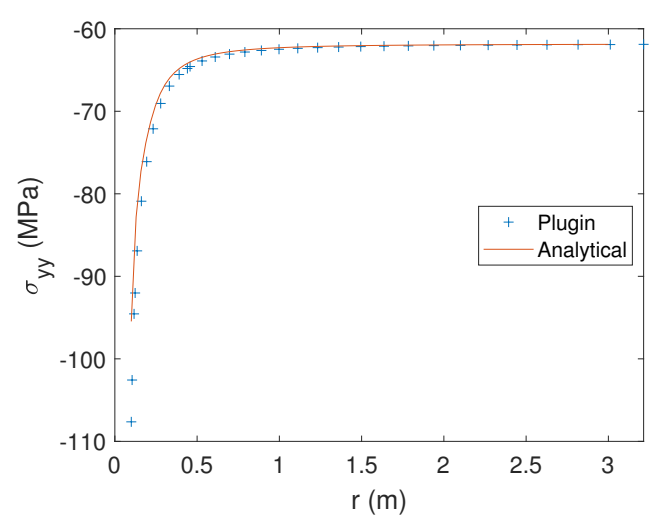

(b) Vertical stresses

Figure 6.15: Total stresses vs radial distance from the center, after $0.1 \mathrm{~s}$

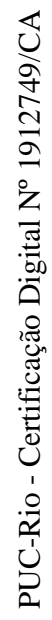

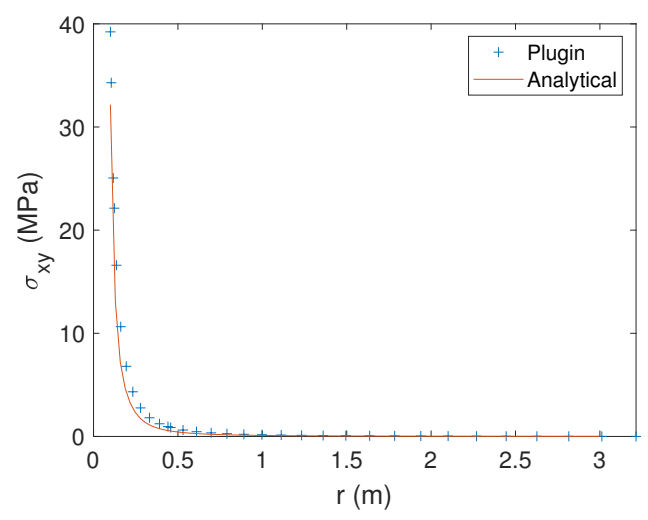

(a) Shear stress

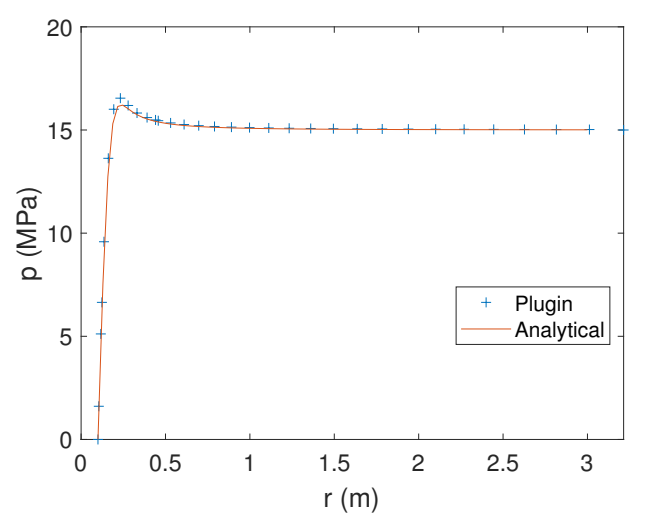

(b) Pore pressure

Figure 6.16: Shear stress and Pore pressure vs radial distance from the center, after $0.1 \mathrm{~s}$ 


\section{Case Study}

This Chapter presents a set of case studies where the simulator computes the ideal mud weight or pressure window. The main goal here is to test the simulator under different conditions as similar as possible to real applications.

\section{1}

\section{Sarvak Formation}

The first problem is based on a case study about the Sarvak reservoir, in the Abadan Plain, Iran [20]. The study presents several experimental data about the three different wells, denoted well A, B and C, including well perforation logs, image logs and rock mechanical test results from vertical plug samples from the region. All the available data was then used to compute the mud weight stability window for each well using the analytical solution and their results were compared with the actual mud weight used during the perforation.

A similar model to the one presented in Figure 6.14 is used here. Figure 7.1a shows the complete model, with a modification to the mesh: it has a refined region around the wellbore, Figure $7.1 \mathrm{~b}$, to better represent the yielded area, while using a coarser T3 mesh to reduce the overall computational cost of this simulation. The model has a total of 9680 nodes and 11076

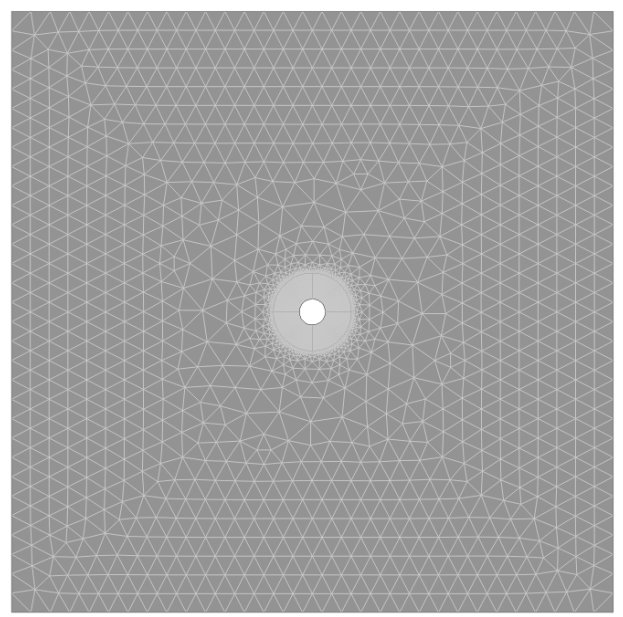

(a) FE model for the Sarvak case study

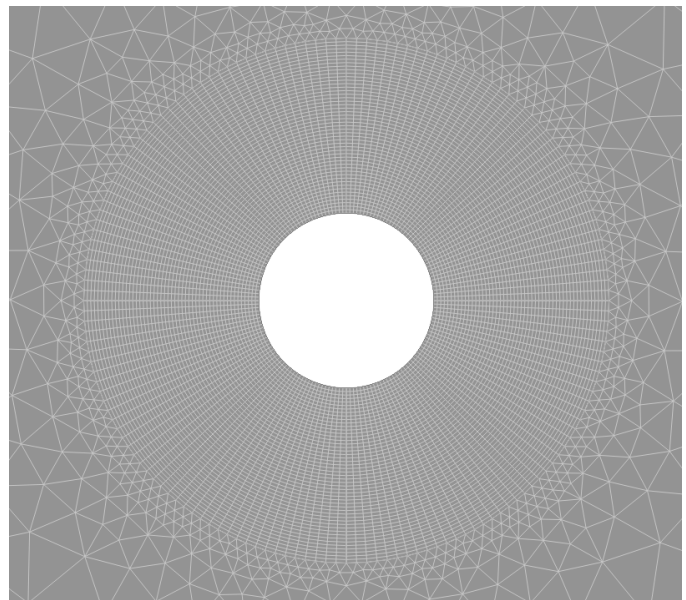

(b) Zoom at the borehole region

Figure 7.1: Sarvak case study model 
elements. Table 7.1 shows the values of each parameter for this model, where the material parameters, stresses and pore pressure field are average values of the experimental data form [20]. Following the hypothesis from [20], no poromechanical coupling was assumed for this model.

\begin{tabular}{ccc}
\hline Parameter & Unit & Value \\
\hline Dimensions $(\mathrm{L})$ & $\mathrm{m}$ & 5 \\
\hline Wellbore Radius $(\mathrm{a})$ & $\mathrm{m}$ & 0.108 \\
\hline Initial Effective Maximum Horizontal Stress $\left(\sigma_{H}^{\prime \prime}\right)$ & $\mathrm{MPa}$ & -36.58 \\
\hline Initial Effective Minimum Horizontal Stress $\left(\sigma_{h}^{\prime \prime}\right)$ & $\mathrm{MPa}$ & -28.80 \\
\hline Initial Effective Vertical Stress $\left(\sigma_{0, v}^{\prime \prime}\right)$ & $\mathrm{MPa}$ & -36.78 \\
\hline Initial pore pressure $\left(p_{0}\right)$ & $\mathrm{MPa}$ & 33.68 \\
\hline Young Modulus $(E)$ & $\mathrm{GPa}$ & 9.55 \\
\hline Poison $(\nu)$ & - & 0.31 \\
\hline Biot $(\alpha)$ & - & 1 \\
\hline Cohesion $(c)$ & $\mathrm{MPa}$ & 7.71 \\
\hline Friction Angle $(\phi)$ & $\circ$ & 39.71 \\
\hline \# Load Steps & - & 5 \\
\hline
\end{tabular}

Table 7.1: Parameters and loads for the Sarvak case study [20]

This problem was solved using the numerical procedure described before to compute the lower limit of the mud weight window. Table 7.2 shows the parameters used as the initial input to define the starting point or initial interval in the stability analysis with the three methods described in chapter 4 (NR, accelerated NR and FP).

\begin{tabular}{ccc}
\hline Parameter & Unit & Value \\
\hline Initial mud pressure $\left(P_{w}\right)$ & $\mathrm{MPa}$ & 32 \\
\hline Initial mud pressure range & $\mathrm{MPa}$ & $34-43$ \\
\hline Limit plastic area $\left(A^{*}\right)$ & $m^{2}$ & 0.002 \\
\hline Tolerance & - & 0.005 \\
\hline
\end{tabular}

Table 7.2: Stability analysis parameters for the Sarvak case study

Table 7.3 presents the results for each method alongside the analytical solution. All three found similar internal pressures and areas. The solution path for both $\mathrm{NR}$ and accelerated was the same, indicating that $A_{2}^{\text {est }} \Delta<<0.25$. 
As for the solution using the FP method, even though it required one more function evaluation, most of the evaluations were from models that remained in the elastic range, hence there wasn't a significant increase in the simulation time. All three analyses resulted in internal pressures approximately 10\% smaller than the analytical result, since the analytical pressure doesn't allow the model to yield.

\begin{tabular}{cccc}
\hline Method & N. f. eval. & Pressure (Pa) & Plastic Area $\left(\mathbf{m}^{2}\right)$ \\
\hline NR & 11 & 38520726.13 & 0.001903 \\
\hline Accelerated NR & 11 & 38520726.13 & 0.001903 \\
\hline FP & 12 & 38466289.18 & 0.001998 \\
\hline Analytical & - & 42367635.27 & - \\
\hline
\end{tabular}

Table 7.3: Results for the Sarvak case study

Figure 7.2 portrays the final plastic area at the solution for the NR analysis. The maximum horizontal stress from Table 7.1 was applied in the $\mathrm{y}$-direction, causing the material to yield perpendicularly at the $\mathrm{x}$-direction. Yielding stats at the wellbore wall at $0^{\circ}$ and $180^{\circ}$ and advances in both circumferential and radial directions.

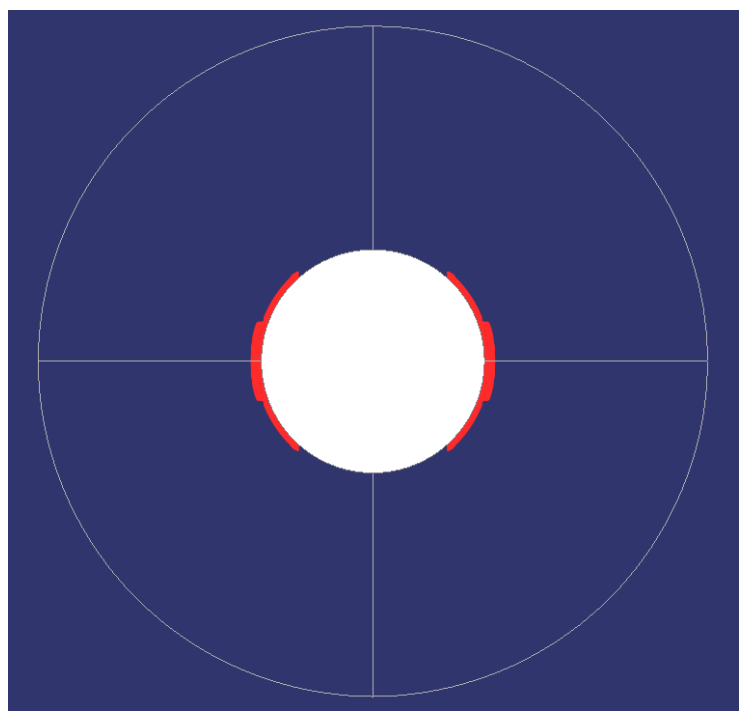

Figure 7.2: Yielded area for the Sarvak case study at the final internal pressure. The red area indicate the zones where yielding occurs.

As for the mud weight, no equivalent depth was informed in [20] corresponding to the average values in 7.1. Assuming that the typical value for the pore pressure gradient under normal formation conditions is around $0.48 \mathrm{psi} / \mathrm{ft}$ (approximately $10.86 \mathrm{kPa} / \mathrm{m}$ ), an equivalent depth for this section of the well would be $3162 \mathrm{~m}$. Using this depth and the final pore pressure from the FP 
method, the required mud weight is $1240.08 \mathrm{~kg} / \mathrm{m}^{3}$ or $77.42 \mathrm{pcf}$, in agreement with the mud weight used during perforation, 82 pcf [20].

\section{2}

\section{Tensile Stress Validation}

This case study aims to compute the ideal mud pressure using the tensile failure condition. It consists of a theoretical model of a vertical well section considering the poromechanical coupling of the medium [60]. The FE model uses a similar mesh to the one in Figure 7.1a, with a refined region near the wellbore, but this time with 16459 nodes and 18154 elements. Tables 7.4 and 7.5 show the parameters used for the numerical model and for the stability analysis, respectively.

\begin{tabular}{|c|c|c|}
\hline Parameter & Unit & Value \\
\hline Dimensions (L) & $\mathrm{m}$ & 6 \\
\hline Wellbore Radius (a) & $\mathrm{m}$ & 0.1 \\
\hline Initial Effective Maximum Horizontal Stress $\left(\sigma_{H}^{\prime \prime}\right)$ & $\mathrm{MPa}$ & -35 \\
\hline Initial Effective Minimum Horizontal Stress $\left(\sigma_{h}^{\prime \prime}\right)$ & $\mathrm{MPa}$ & -20 \\
\hline Initial Effective Vertical Stress $\left(\sigma_{0, v}^{\prime \prime}\right)$ & $\mathrm{MPa}$ & -40 \\
\hline Initial pore pressure $\left(p_{0}\right)$ & $\mathrm{MPa}$ & 15 \\
\hline Young Modulus $(E)$ & $\mathrm{GPa}$ & 14.4 \\
\hline Poison $(\nu)$ & - & 0.2 \\
\hline $\operatorname{Biot}(\alpha)$ & - & 0.222 \\
\hline Cohesion $(c)$ & $\mathrm{MPa}$ & 10 \\
\hline Friction Angle $(\phi)$ & $\circ$ & 35 \\
\hline Permeability $(k)$ & $\mathrm{mD}$ & 10 \\
\hline Porosity $(n)$ & - & 0.2 \\
\hline Viscosity $(\mu)$ & $\mathrm{Pa} \mathrm{s}$ & $10^{-3}$ \\
\hline Grain Compressibility $\left(K_{s}\right)$ & $\mathrm{GPa}$ & 36 \\
\hline Fluid Compressibility $\left(K_{w}\right)$ & $\mathrm{GPa}$ & 2.8 \\
\hline Total time & $\mathrm{h}$ & 12 \\
\hline
\end{tabular}

Table 7.4: Parameters and loads for the tensile failure case study [60] 


\begin{tabular}{ccc}
\hline Parameter & Unit & Value \\
\hline Initial mud pressure $\left(P_{w}\right)$ & $\mathrm{MPa}$ & 35 \\
\hline Initial mud pressure range & $\mathrm{MPa}$ & $15-43$ \\
\hline Tensile Resistance $\left(T_{0}\right)$ & $\mathrm{MPa}$ & 0 \\
\hline Tolerance & - & $10^{-7}$ \\
\hline
\end{tabular}

Table 7.5: Stability analysis parameters for the tensile failure case study

The numerical results are presented in Table 7.6. It also shows the result of the analytical solution for comparison, even though the analytical solution doesn't account for the poromechanical coupling. In this simulation, the results for the numerical and the analytical solution were almost identical, indicating that the poromechanical coupling had only a minor influence on the results. Furthermore, all three methods yielded the same internal pressure, most likely due to the model remaining in the elastic range at the solution.

\begin{tabular}{cccc}
\hline Method & N. f. eval. & Pressure $(\mathbf{P a})$ & $\sigma_{1}^{\prime \prime}(\mathbf{P a})$ \\
\hline NR & 11 & 28168314.84 & $-5.65 \mathrm{e}-06$ \\
\hline Accelerated NR & 9 & 28168314.84 & $7.64 \mathrm{e}-07$ \\
\hline FP & 11 & 28168314.84 & $1.80 \mathrm{e}-06$ \\
\hline Analytical & - & 28330000 & - \\
\hline
\end{tabular}

Table 7.6: Results for the tensile failure case study

Figure 7.3 shows the final maximum effective principal stress around the wellbore at the end of the simulation. As expected, the maximum effective stress appears at the wellbore wall, at the direction of the maximum horizontal stress.

\section{3}

\section{Gulf of Mexico}

The following case study reproduces a common challenge in the Gulf of Mexico (GoM) region and other offshore drilling operations: the stress perturbations caused by nearby salt bodies. The presence of a salt body has a significant influence on the surrounding areas as it redistributes the loads to the neighboring regions. In [80], the authors created several models with idealized salt geometries, e.g. spherical, sheet and column, found in the GoM region to compute the modified stress state. Each model uses the gravitational load to 


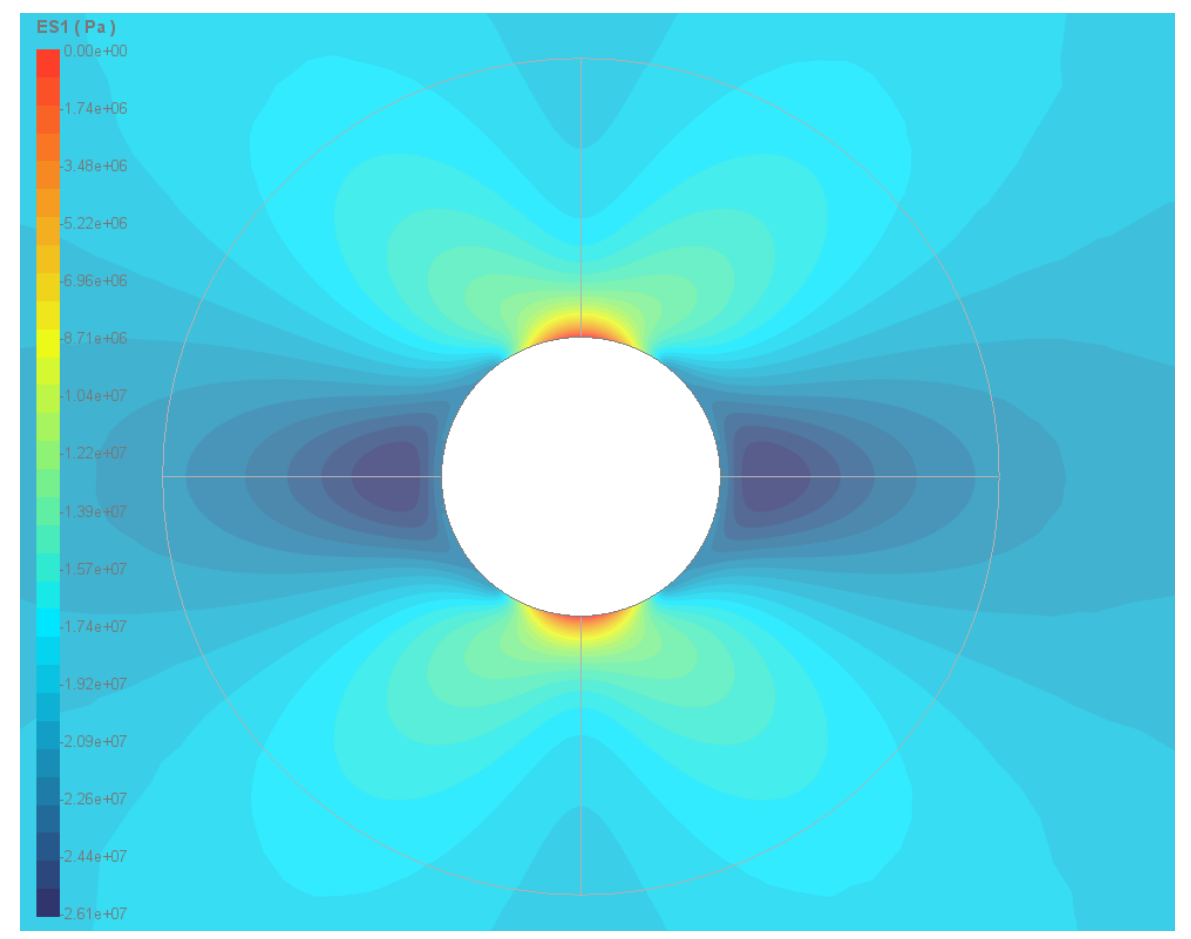

Figure 7.3: Maximum principal effective stress at the end of the analysis

calculate the initial vertical stress and, using the known far-field stress ratios ${ }^{1}$, the horizontal stresses. Then, the model is "released" to let the viscous behavior of the salt take place. The inelastic deformation of the salt redistributes the stresses at the adjacent regions until the deviatoric stresses (or the Mises equivalent stress) in the salt reach a small value. At this point, the viscous strains of the salt become minimal and the stresses reach its final value [80].

In this work, the plugin simulator computes the minimum mud pressure of an inclined well located near the spherical salt body from [80]. The model assumes that the material follows the MC criterion and has poromechanical fluid mechanical coupling. Table 7.7 presents the geometry, loads and material parameters for this simulation. The elastic material parameters are the same as the ones from [80], whereas the yield model and other material parameters required for the fluid flow analysis are from [81]. The model permeability was estimated from a permeability-porosity trend, depicted in Figure 7.4, from a case study of a nearby field in the GoM [82]. The wellbore section model has 20327 nodes and 22050 elements.

\begin{tabular}{ccc}
\hline Parameter & Unit & Value \\
\hline Dimensions (L) & $\mathrm{m}$ & 6 \\
\hline Wellbore Radius (a) & $\mathrm{m}$ & 0.1 \\
\hline
\end{tabular}

${ }^{1}$ ratio between the horizontal and vertical stresses 
Continuation of Table 7.7

\begin{tabular}{|c|c|c|}
\hline Parameter & Unit & Value \\
\hline Well Inclination & $\circ$ & 60 \\
\hline Minimum Horizontal Stress Azimuth & $\circ$ & 90 \\
\hline Initial Effective Maximum Horizontal Stress $\left(\sigma_{H}^{\prime \prime}\right)$ & $\mathrm{MPa}$ & -57.29 \\
\hline Initial Effective Minimum Horizontal Stress $\left(\sigma_{h}^{\prime \prime}\right)$ & $\mathrm{MPa}$ & -87.99 \\
\hline Initial Effective Vertical Stress $\left(\sigma_{0, v}^{\prime \prime}\right)$ & $\mathrm{MPa}$ & -103.79 \\
\hline Initial pore pressure $\left(p_{0}\right)$ & $\mathrm{MPa}$ & 68.2 \\
\hline Young Modulus $(E)$ & GPa & 9.5 \\
\hline Poison $(\nu)$ & - & 0.25 \\
\hline Biot $(\alpha)$ & - & 0.3667 \\
\hline Cohesion $(c)$ & $\mathrm{MPa}$ & 2.5 \\
\hline Friction Angle $(\phi)$ & $\circ$ & 30 \\
\hline Permeability $(k)$ & $\mathrm{mD}$ & 1 \\
\hline Porosity $(n)$ & - & 0.154 \\
\hline Viscosity $(\mu)$ & Pas & $10^{-3}$ \\
\hline Grain Compressibility $\left(K_{s}\right)$ & GPa & 10 \\
\hline Fluid Compressibility $\left(K_{w}\right)$ & $\mathrm{GPa}$ & 2.25 \\
\hline Total time & $\mathrm{h}$ & 24 \\
\hline
\end{tabular}

Table 7.7: Parameters and loads for the GoM case study [80, 81, 82]

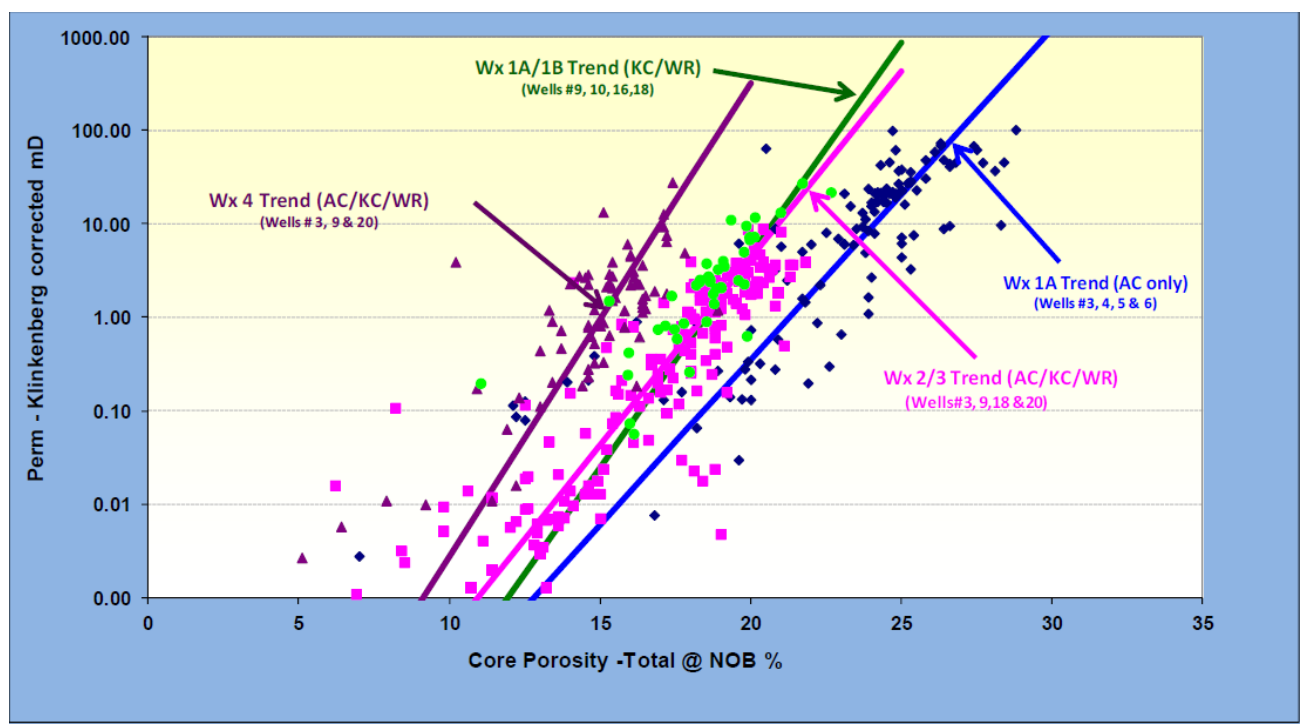

Figure 7.4: Permeability vs Porosity trend [82] 
Table 7.8 presents the parameters related to the wellbore stability analysis. The initial mud pressure for the NR and accelerated NR solutions is an estimative based on the far-field pore pressure, with a suitable maximum value for the mud pressure range. The model uses the same limit plastic area as the first case due to the similar well radius.

\begin{tabular}{ccc}
\hline Parameter & Unit & Value \\
\hline Initial mud pressure $\left(P_{w}\right)$ & $\mathrm{MPa}$ & 68 \\
\hline Initial mud pressure range & $\mathrm{MPa}$ & $68-80$ \\
\hline Limit plastic area $\left(\mathrm{A}^{*}\right)$ & $\mathrm{m}^{2}$ & 0.002 \\
\hline Tolerance & - & 0.004 \\
\hline
\end{tabular}

Table 7.8: Stability analysis parameters for the GoM case study

The results for this simulation are presented in Table 7.9. Again, all three analyses reached similar mud pressures and yielded zones. Once more, the solution path for the NR accelerated method was the same as the NR one, but this time the FP method required only $2 / 3$ of the function evaluations if compared to the NR method for this combination of initial mud pressure, initial mud pressure range and tolerance. Unlike previous cases, there is no analytical closed-form solution for this problem to compare with the numerical counterparts.

\begin{tabular}{cccc}
\hline Method & N. f. eval. & Pressure $(\mathbf{P a})$ & Plastic Area $\left(\mathbf{m}^{2}\right)$ \\
\hline NR & 9 & 72700828.2 & 0.002 \\
\hline Accelerated NR & 9 & 72700828.2 & 0.002 \\
\hline FP & 6 & 72710754.64 & 0.00199854 \\
\hline
\end{tabular}

Table 7.9: Results for the GoM case study

\section{4}

\section{Espírito Santo Basin}

The final case aims to study a wellbore instability issue reported for the well 3-BRSA-1229-ESS, at block BM-ES-22A, Espírito Santo Basin, Brazil. It was part of an offshore operation to explore and evaluate a subsalt reservoir. The wellbore project had four drilling stages: two initial stages before reaching the salt body, a third stage at the salt body and the last one after the salt body. During the third and fourth stages, the operators faced significant difficulties, such as rate of penetration reduction and risk of stuck pipe in the salt, and 
presence of shear failure zones in the subsalt region [69].

In [69], the author used Tectos to reproduce the experimental data from well logs and study the slat influence in the subsalt zone stresses. Figure 7.5 shows the FE model created from seismic images of the region. It consists of three distinct zones: the salt region (in pink), a subsalt region, where the material has an elastoplastic behavior, and a third elastic zone in the rest of the model. The yellow and blue lines indicate well 3-BRSA-1229-ESS and the water level, respectively.

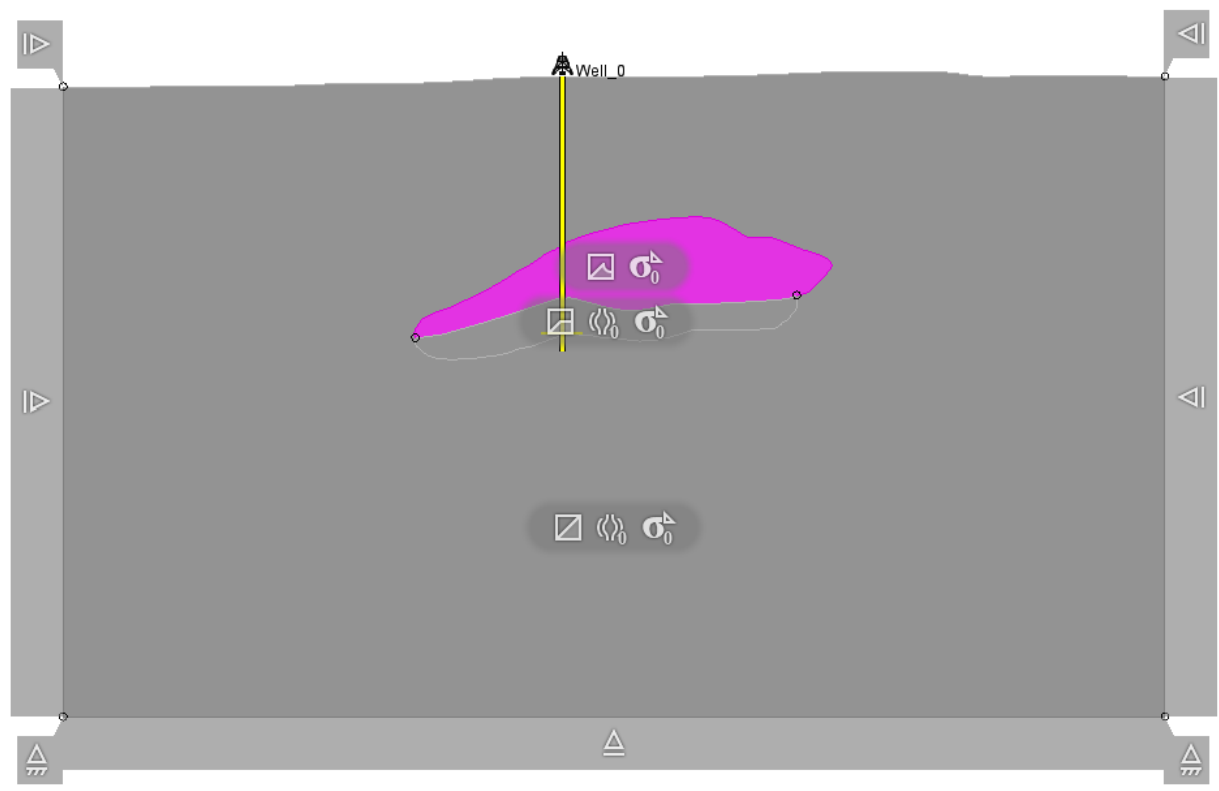

Figure 7.5: FE model from the seismic image [69]

This model presents some unique features if compared to the previous ones discussed here. Some material parameters of the elastic and elastoplastic zones, namely the Young modulus, Poisson coefficient, Biot coefficient and bulk density, have spatial distributions related to the depth. Here, each parameter is assumed to be a linear function of the depth with the linear coefficients extracted from the data in [69]. Furthermore, the elastoplastic behavior in the subsalt zone was modeled using two yield criteria, the MC and MCC models, but only the former will be considered for the numerical analysis. The MC model will be used to provide an analytical solution for comparison. As for the salt layer, the Double Mechanism (DM) creep law describes its viscous behavior. A mathematical description of the DM law is detailed in $[83,84,85,86]$, where the authors used the DM model to reproduce the 
behavior of halite salt rock found in the Espírito Santo basin. The numerical procedure to consider the viscous forces is described in $[87,88]$.

Following the procedure discussed in the previous case study, an initial analysis in Tectos computes the vertical stresses from the gravitational and water column loads. Then, a second analysis, also using Tectos and Ghem, uses the effective stress ratio to compute the horizontal stresses and the model is released for the viscous effects to take place. Tables 7.10 and 7.11 presents the parameters for the two elastoplastic models. Equation (7-1) provides the initial ellipsis radius for the MCC model since the initial radius isn't available. For more information regarding the material parameters and loads for the FE model, the reader is referred to [69].

$$
a_{0}=\frac{\frac{q^{2}}{M^{2}|p|}+|p|+p_{t}}{1+\beta}
$$

\begin{tabular}{ccc}
\hline Parameter & Unit & Value \\
\hline Virgin Compression Line slope $(\lambda)$ & - & 0.131 \\
\hline Swelling Line slope $(\kappa)$ & - & 0.011 \\
\hline Critical State Line slope $(M)$ & - & 0.750 \\
\hline Initial void ratio $\left(e_{0}\right)$ & - & 0.430 \\
\hline
\end{tabular}

Table 7.10: MCC criterion parameters [69]

\begin{tabular}{ccc}
\hline Parameter & Unit & Value \\
\hline Cohesion $(c)$ & $\mathrm{MPa}$ & 2.59 \\
\hline Friction Angle $(\phi)$ & $\circ$ & 19.4 \\
\hline
\end{tabular}

Table 7.11: MC criterion parameters [69]

Figure 7.6 presents the VM equivalent stress for the model after a hundred years. At this point, the VM stress was less than $1 \mathrm{MPa}$, which is considered sufficiently small [80]. Figure 7.7 on the other hand shows which parts of the elastoplastic zone developed plastic strains. Even though a short section of the well path is still inside the elastic domain, the material at that region remains close to yielding. 


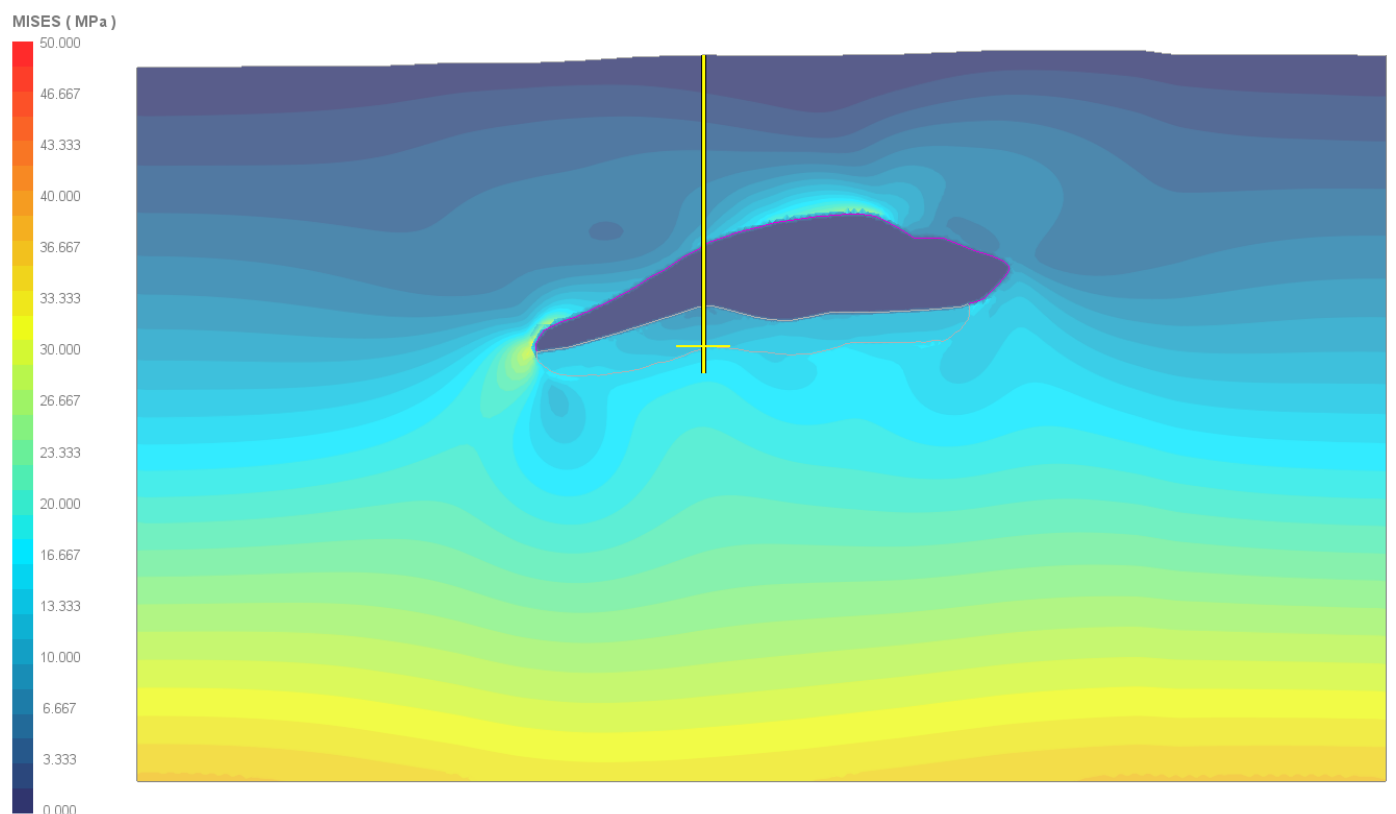

Figure 7.6: VM equivalent stress after a hundred years

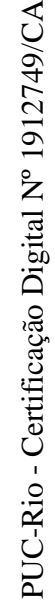

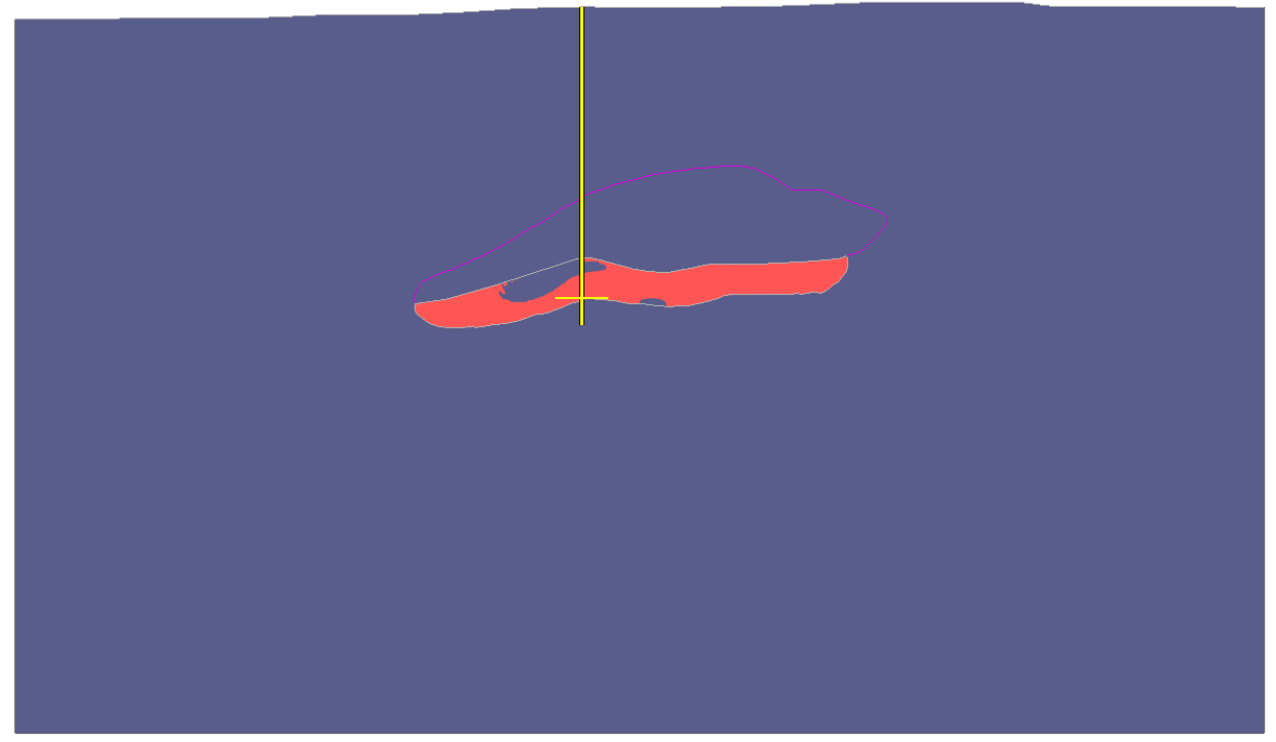

Figure 7.7: Yielded zone after a hundred years 
The horizontal yellow line in Figures 7.6 and 7.7 indicates the wellbore section used for the following stability analysis. The section uses the stresses and material properties results from the initial analysis as input parameters. The mesh in this model has 17000 nodes and 18756 elements. Table 7.12 shows the complete list of parameters related to the wellbore section model. The permeability, porosity and both grain and fluid compressibilities are not specified in [69]. Here, both permeability and porosity were taken from a case study of a nearby reservoir [89], while the compressibilities were estimated from the material database of Tectos. As for the hole radius, since the well used a $95 / 8$ in $+97 / 8$ in casing, it is assumed that the drilling tool diameter is 12.25 in [56]. The wellbore section model has 20327 nodes and 22050 elements.

\begin{tabular}{ccc}
\hline Parameter & Unit & Value \\
\hline Dimensions $(\mathrm{L})$ & $\mathrm{m}$ & 9 \\
\hline Wellbore Radius $(\mathrm{a})$ & $\mathrm{m}$ & 0.1555 \\
\hline TVD & $\mathrm{m}$ & 4440 \\
\hline Initial Effective Maximum Horizontal Stress $\left(\sigma_{H}^{\prime \prime}\right)$ & $\mathrm{MPa}$ & -24.693 \\
\hline Initial Effective Minimum Horizontal Stress $\left(\sigma_{h}^{\prime \prime}\right)$ & $\mathrm{MPa}$ & -24.286 \\
\hline Initial Effective Vertical Stress $\left(\sigma_{0, v}^{\prime \prime}\right)$ & $\mathrm{MPa}$ & -36.312 \\
\hline Initial pore pressure $\left(p_{0}\right)$ & $\mathrm{MPa}$ & 43.620 \\
\hline Young Modulus $(E)$ & $\mathrm{GPa}$ & 16.631 \\
\hline Poison $(\nu)$ & - & 0.368 \\
\hline Biot $(\alpha)$ & - & 0.65 \\
\hline Ellipsis radius $(a)$ & $\mathrm{MPa}$ & 19.3 \\
\hline Permeability $(k)$ & $\mathrm{mD}$ & 270 \\
\hline Porosity $(n)$ & - & 0.247 \\
\hline Viscosity $(\mu)$ & $\mathrm{Pas}$ & 0.001 \\
\hline Train Compressibility $\left(K_{s}\right)$ & $\mathrm{GPa}$ & 60 \\
\hline Fluid Compressibility $\left(K_{w}\right)$ & $\mathrm{GPa}$ & 2.250 \\
\hline Total time & 12 \\
\hline
\end{tabular}

Table 7.12: Parameters and loads for the wellbore section $[69,89]$

Table 7.13 presents the values used for the stability analysis. The plastic area criterion is used to compute the lower and upper limits of the mud pressure window. The stability analysis with the tensile resistance is omitted here because the simulator did not find a solution even at a much higher 
pressure than the one found with the plastic area criterion.

\begin{tabular}{ccc}
\hline Parameter & Unit & Value \\
\hline Initial mud pressure - lower limit $\left(P_{w}\right)$ & $\mathrm{MPa}$ & 48 \\
\hline Initial mud pressure range - lower limit & $\mathrm{MPa}$ & $48-52$ \\
\hline Initial mud pressure - upper limit $\left(P_{w}\right)$ & $\mathrm{MPa}$ & 58 \\
\hline Initial mud pressure range - upper limit & $\mathrm{MPa}$ & $55-59$ \\
\hline Limit plastic area $\left(\mathrm{A}^{*}\right)$ & $\mathrm{m}^{2}$ & 0.004805 \\
\hline Tolerance & - & 0.0005 \\
\hline
\end{tabular}

Table 7.13: Stability analysis parameters for the wellbore stability analysis

Tables 7.14 and 7.15 shows the results for the lower and upper limits. In both cases, all three methods reached similar internal pressures, but the plastic area for both NR and accelerated NR resulted in a final area approximately $8 \%$ smaller than the required value. This indicates that the plastic area in Equation 4-9 is extremely sensitive to variations in the internal pressure for the material parameters and loads in this model. In this case, numerical approximation errors influence the derivative from the finite difference method and may deteriorate NR results. Nevertheless, all methods required the same number of function evaluations for the specified initial point, mud pressure range and tolerance.

\begin{tabular}{cccc}
\hline Method & N. f. eval. & Pressure (MPa) & Plastic Area $\left(\mathrm{m}^{2}\right)$ \\
\hline NR & 9 & 51247360.56 & 0.00444778341 \\
\hline Accelerated NR & 9 & 51247360.56 & 0.00444778341 \\
\hline FP & 9 & 51236054.03 & 0.0047912 \\
\hline
\end{tabular}

Table 7.14: Results for the lower limit of the mud pressure window

\begin{tabular}{cccc}
\hline Method & N. f. eval. & Pressure $(\mathbf{P a})$ & Plastic Area $\left(\mathbf{m}^{2}\right)$ \\
\hline NR & 11 & 55739269.02 & 0.004821167966 \\
\hline Accelerated NR & 11 & 55739269.02 & 0.004821167966 \\
\hline FP & 11 & 55738396.44 & 0.00479926476 \\
\hline
\end{tabular}

Table 7.15: Results for the upper limit of the mud pressure window

Figures 7.8 and 7.9 compare the numerical results from the NR method with the analytical solution and the MC parameters from Table 7.11. The 
numerical solution provided a much narrower window than the analytical one, indicating the project could be more challenging than the analytical solution may suggest. The results also demonstrate the combined influence of a different yield criterion, in this case, a capped instead of an uncapped criterion, and the influence of the poromechanical coupling in the results. Using the TVD for the wellbore section from Table 7.12, the final mud weight window is $9.76 \mathrm{ppg}$ to $10.62 \mathrm{ppg}$, whereas the actual mud window available in the well log is 9.7 ppg to 12ppg [69]. The actual values used during the operation in that region were initially $10.4 \mathrm{ppg}$ and then 11ppg. Even though the numerical result was similar to the project values, these results depend on the limit plastic area and material parameters, which were not available directly for that region.

Figure 7.9 also presents a few points of the solution path for the mud window, with an "x" marker, and the solution, with a "*" marker. The window starts with a wider range and, in this case, converges to the narrower mud pressure interval. The difference between the two predictions shows the influence of the poromechanical coupling and the MCC yield criterion in the final mud window.

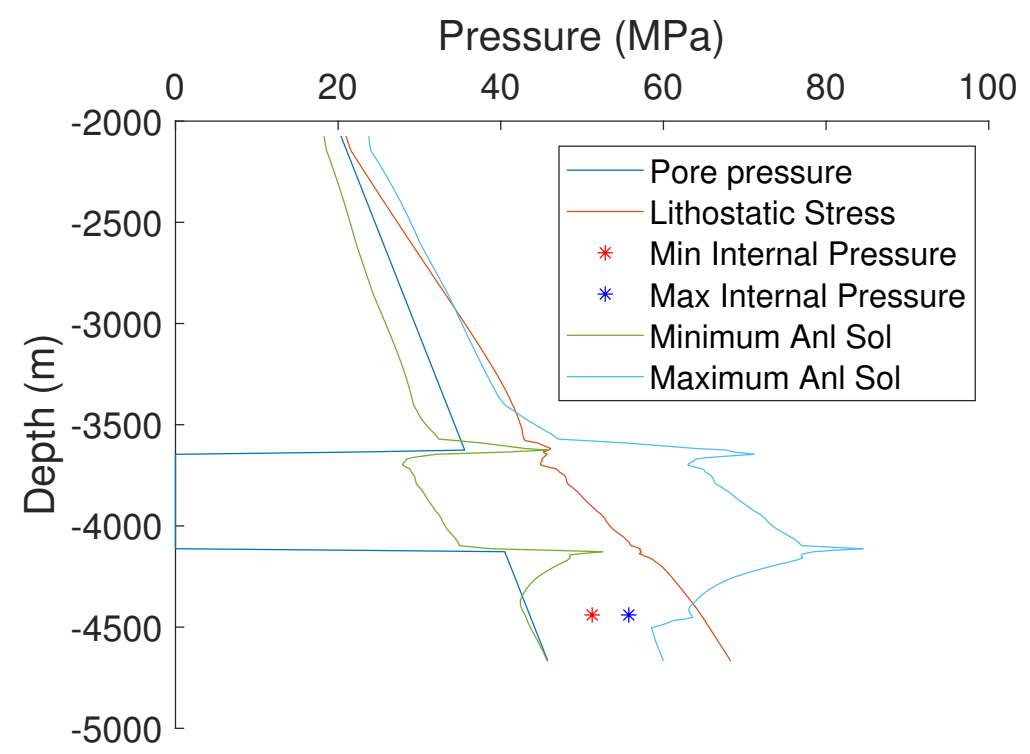

Figure 7.8: Mud pressure window 


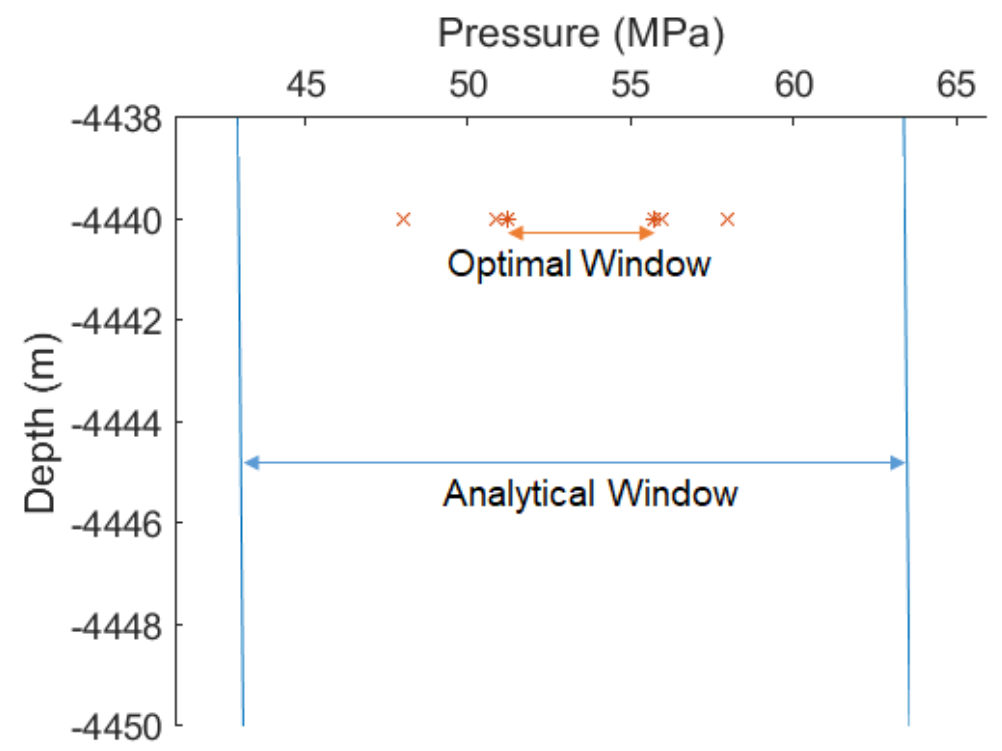

Figure 7.9: Zoom at the wellbore section depth 


\section{8 \\ Conclusion}

This thesis presented a novel simulator to perform wellbore stability analysis and to compute the optimal mud pressure window. The simulator utilized two different equations to describe the wellbore failure and calculated the required mud pressure for each equation utilizing root-finding algorithms. During the solution process, the simulator used the FEM to solve the required geomechanical analyses. At the end of the process, the simulator informed the user of the desired mud pressure according to each condition, as well as the displacements, strains, stresses and pore pressures around the wellbore.

The initial part of this work described the theory used to model the geomechanical problem. The model assumed the rock formation was a deformable porous medium. For simplicity, it presented isotropic linear elastic behavior according to Hooke's Law until a limit stresses state described by a suitable yield function, e.g., VM, MC and MCC criteria. If stressed any further, it would start to develop plastic strains. The medium was also assumed fully saturated with a single-phase Newtonian fluid and Darcy's law related the pore pressure gradients to the flow velocities. Biot's theory described the interaction between the pore pressure and the effective stresses. Both mechanical and fluid flow equations yielded a coupled partial differential equation system in terms of the porous matrix displacement and pore pressure.

Here, we used the FEM to solve the coupled system. The method divided the domain into elements and utilized the nodal values to approximate the displacement and pore pressure fields. The material elastoplastic behavior was treated as a local problem, at each Gauss point. Hence, if the material yielded for a given displacement and pore pressure increment, the simulator used the CPA to update the stresses such that the final stress state obeys the desired yield function. Therefore, the solution procedure consisted of solving a matrix system to compute displacement and pore pressure updates and then utilizing the CPA to find the new stresses at each element. Instead of directly solving the full matrix system, we used a Staggered Solution Procedure to divide the fully coupled simulation into two problems: a mechanical and a fluid flow one. With this strategy, the indefinite coupled matrix system becomes two smaller positive definite matrices and now the nonlinearity was only present in the 
mechanical analysis.

As for the wellbore stability analysis, we considered two approaches to evaluating the required mud pressure: an analytical closed-form and a numerical solution. The former used known elasticity solutions to compute the stresses around the wellbore and rewrote the MC criterion at $\Phi=0$ or at the tensile cutoff in terms of the in-situ stresses to calculate the limit mud pressure for a vertical well. The latter generalized the failure condition for the well with the total plastic zone area around the wellbore or for the maximum effective stress to compute the mud pressure. Then, a root-finding algorithm iteratively solved a sequence of geomechanical analyses to find the optimal mud pressure. In this approach, the wellbore stability analysis was not as limited to model simplifications and could better reproduce the physical behavior of the medium around the wellbore.

All the methods and equations previously discussed were implemented in a novel simulator based on a plugin architecture. It provided a more flexible environment to develop the software to include several methods and solve different analyses. In this work, the simulator could run both FE or wellbore stability analyses assuming multiple conditions (e.g., poromechanical coupling, different yield criteria, elastic or elastoplastic behavior, different element types) by adding or exchanging specific modules in a plugin scheme. The plugin framework also allowed other third-party plugins to be included in the scheme, making the simulator even more flexible.

The final part presented several validation examples and case studies to test the plugin simulator. The simulator was able to reproduce a series of analytical solutions for simplified problems. It also successfully estimated the ideal mud pressure and mud weight for four case studies under different conditions. All results were within an acceptable range from the analytical solution or the actual mud pressure and weight, when available. As for the root-finding methods used to find the numerical solution, both FP method and the combination of the NR method with the finite difference scheme produced similar results in terms of the final mud pressure and number of function evaluations required to reach the solution. The accelerated NR method required only a small modification to be implemented, but was only able to provide a reduction in the number of function evaluations in one of the four case studies presented here.

\section{1}

\section{Future Works}

Suggestions for future works include: 
- Thermal coupling. The presence of thermal stresses greatly influences the final state at the wellbore. By adding the thermo-poromechanical coupling to the problem, the simulator can consider other relevant parameters in the stability analysis, namely the mud temperature;

- Generalized Plane Strain. In this work, each stability analysis uses a section under plane strain conditions. If a generalized plane strain condition is used instead, the simulator also consider out-of-plane shear stresses, especially relevant for inclined wellbore sections;

- Different Return Mapping algorithms and yield criteria. Only a few yield criteria and one return mapping algorithm were implemented here. Other yield criteria, for example, weak plane and triaxial criteria, together with suited return mapping algorithms may result in better predictions for certain models and/or faster simulations;

- Dual Porosity Model, multiphase flow and variable permeability and porosity. The simulator assumes the porous medium is fully saturated with a single-phase fluid. This hypothesis allows for the initial poromechanical analyses, but it may be too simplistic for some geomechanical analyses in regions where the medium may not be fully saturated or may be fractured;

- Low Permeability. Low permeability models represent a numerical challenge and often require changes in the formulation or element type. New element plugin schemes could be implemented to better simulate these situations;

There are two main objectives behind these suggestions. First, it will allow the simulator to reproduce a wider range of real cases and consequently more stability analyses. Second, it will explore the flexibility of a plugin framework.

Furthermore, the present simulator may also be used to study other geomechanical phenomena, such as the influence of different $i n$-situ stress states in the final breakout area or the use of other failure criteria to characterize wellbore failure. 


\section{Bibliography}

\section{References}

[1] YORK, P.; PRICHARD, D.; DODSON, J.; DODSON, T.; ROSENBERG, S.; GALA, D. ; UTAMA, B.. Eliminating non-productive time associated with drilling through trouble zones. In: OTC OFFSHORE TECHNOLOGY CONFERENCE, 05 2009. OTC-20220-MS.

[2] GHOLAMI, R.; AADNOY, B.; FOON, L. Y. ; ELOCHUKWU, H.. A methodology for wellbore stability analysis in anisotropic formations: A case study from the canning basin, western australia. Journal of Natural Gas Science and Engineering, 37:341 - 360, 2017.

[3] EXPLORATION, O. I. I.; FORUM, P.. Environmental Management in Oil and Gas Exploration and Production: An Overview of Issues and Management Approaches. E \& P forum report // Oil Industry International Exploration and Production Forum. E\&P Forum, 1997.

[4] LI, S.; GEORGE, J. ; PURDY, C.. Pore-Pressure and WellboreStability Prediction To Increase Drilling Efficiency. Journal of Petroleum Technology, 64(02):98-101, 2012.

[5] PAŠIĆ, B.; GAURINA-MEDIMUREC, N. ; MATANOVIĆ, D.. Wellbore instability: Causes and consequences. Rudarsko Geolosko Naftni Zbornik, 19:87-98, 2007.

[6] MOOS, D.; PESKA, P.; FINKBEINER, T. ; ZOBACK, M.. Comprehensive wellbore stability analysis utilizing quantitative risk assessment. Journal of Petroleum Science and Engineering, 38(3):97 - 109, 2003. Borehole Stability.

[7] MA, T.; CHEN, P.; YANG, C. ; ZHAO, J.. Wellbore stability analysis and well path optimization based on the breakout width model and mogi-coulomb criterion. Journal of Petroleum Science and Engineering, 135:678-701, 2015. 
[8] AL-AJMI, A. M.; ZIMMERMAN, R. W.. A new well path optimization model for increased mechanical borehole stability. Journal of Petroleum Science and Engineering, 69(1):53-62, 2009.

[9] DIVENYI, S.; SAVI, M. A.; WIERCIGROCH, M. ; PAVLOVSKAIA, E.. Drillstring vibration analysis using non-smooth dynamics approach. Nonlinear Dynamics, 70(2):1017-1035, Oct 2012.

[10] DE MORAES, L. P.; SAVI, M. A.. Drill-string vibration analysis considering an axial-torsional-lateral nonsmooth model. Journal of Sound and Vibration, 438:220-237, 2019.

[11] ZHANG, S.; WANG, H.; QIU, Z.; CAO, W.; HUANG, H. ; CHEN, Z.. Calculation of safe drilling mud density window for shale formation by considering chemo-poro-mechanical coupling effect. Petroleum Exploration and Development, 46(6):1271-1280, 2019.

[12] BATHE, K.. Finite Element Procedures. Prentice Hall, 2nd edition, 2006.

[13] LEWIS, R.; SCHREFLER, B.. The Finite-Element Method in Deformation and Consolidation of Porous Media, volume 3. John Wiley \& Sons, Ltd, 011987.

[14] NETO, E. D. S.; PERIĆ, D. ; OWEN, D.. Computational methods for plasticity : theory and applications. John Wiley \& Sons, Ltd, 2008.

[15] OWEN, D.; HINTON, E.. Finite Elements in Plasticity: Theory and Practice. Pineridge Press, 1980.

[16] BORJA, R. I.. Plasticity. Springer Berlin Heidelberg, Berlin, Heidelberg, 2013.

[17] MISES, R. V.. Mechanik der festen körper im plastisch- deformablen zustand. Nachrichten von der Gesellschaft der Wissenschaften zu Göttingen, Mathematisch-Physikalische Klasse, p. 11, 1913.

[18] LABUZ, J. F.; ZANG, A.. Mohr-Coulomb failure criterion. Rock Mechanics and Rock Engineering, 45(6):975-979, 2012.

[19] ABBO, A.; SLOAN, S.. A smooth hyperbolic approximation to the mohr-coulomb yield criterion. Computers \& Structures, 54(3):427 441, 1995. 
[20] EZATI, M.; AZIZZADEH, M.; RIAHI, M. A.; FATTAHPOUR, V. ; HONARMAND, J.. Wellbore stability analysis using integrated geomechanical modeling: a case study from the sarvak reservoir in one of the sw iranian oilfields. Arabian Journal of Geosciences, 13, 022020.

[21] AL-AJMI, A.; ZIMMERMAN, R.. Stability analysis of vertical boreholes using the mogi-coulomb failure criterion. International Journal of Rock Mechanics and Mining Sciences, 43(8):1200 - 1211, 2006.

[22] DRUCKER, D. C.; PRAGER, W.. Soil mechanics and plastic analysis or limit design. Quarterly of Applied Mathematics, 10:157-165, 1952.

[23] NAYAK, G. C.; ZIENKIWICZ, O. C.. Convenient Form of Stress Invariants for Plasticity. Journal of the Structural Division, 98(4):949954, 1972.

[24] SLOAN, S. W.; BOOKER, J. R.. Removal of singularities in tresca and mohr-coulomb yield functions. Communications in Applied Numerical Methods, 2(2):173-179, 1986.

[25] ABBO, A.; LYAMIN, A.; SLOAN, S. ; HAMBLETON, J.. A c2 continuous approximation to the mohr-coulomb yield surface. International Journal of Solids and Structures, 48(21):3001 - 3010, 2011.

[26] BRUNO, H.; BARROS, G.; MENEZES, I. ; MARTHA, L.. Returnmapping algorithms for associative isotropic hardening plasticity using conic optimization. Applied Mathematical Modelling, 78, 102019.

[27] AL-AJMI, A.; ZIMMERMAN, R.. Relation between the mogi and the coulomb failure criteria. International Journal of Rock Mechanics and Minings Sciences, 42(3):431-439, Apr. 2005.

[28] PERIĆ, D.. Analytical solutions for a three-invariant cam-clay model subjected to drained loading histories. International Journal for Numerical and Analytical Methods in Geomechanics, 30(5):363-387, 2006.

[29] BORJA, R. I.; LEE, S. R.. Cam-clay plasticity, part 1: Implicit integration of elasto-plastic constitutive relations. Computer Methods in Applied Mechanics and Engineering, 78(1):49-72, 1990.

[30] ROCSCIENCE. Rs3 sofware manual, 2016.

[31] CUI, L.; KALIAKIN, V.; ABOUSLEIMAN, Y. ; CHENG, A.-D.. Finite element formulation and application of poroelastic generalized 
plane strain problems. International Journal of Rock Mechanics and Mining Sciences, 34(6):953 - 962, 1997.

[32] BAI, M.; ABOUSLEIMAN, Y.; CUI, L. ; ZHANG, J.. Dual-porosity poroelastic modeling of generalized plane strain. International Journal of Rock Mechanics and Mining Sciences, 36(8):1087 - 1094, 1999.

[33] ZHANG, J.; BAI, M. ; ROEGIERS, J.-C.. Dual-porosity poroelastic analyses of wellbore stability. International journal of rock mechanics and mining sciences, 40(4):473-483, 2003.

[34] MENG, M.; BALDINO, S.; MISKA, S. ; TAKACH, N.. Wellbore stability in naturally fractured formations featuring dual-porosity/singlepermeability and finite radial fluid discharge. Journal of Petroleum Science and Engineering, 174:790 - 803, 2019.

[35] MÜLlER, A. L.; DO AMARAL VARGAS, E.; VAZ, L. E.; FIGUEIREDO, R. P. ; GONÇALVES, C. J.. Numerical analysis of sand/solids production in boreholes considering fluid-mechanical coupling in a cosserat continuum. International Journal of Rock Mechanics and Mining Sciences, 48(8):1303-1312, 2011.

[36] SCHREFLER, B. A.; SCOTTA, R.. A fully coupled dynamic model for two-phase fluid flow in deformable porous media. Computer Methods in Applied Mechanics and Engineering, 190(24):3223 - 3246, 2001.

[37] VERMEER, P. A.; VERRUIJT, A.. An accuracy condition for consolidation by finite elements. International Journal for Numerical and Analytical Methods in Geomechanics, 5(1):1-14, 1981.

[38] REED, M. B.. An investigation of numerical errors in the analysis of consolidation by finite elements. International Journal for Numerical and Analytical Methods in Geomechanics, 8(3):243-257, 1984.

[39] MURAD, M. A.; LOULA, A. F. D.. On stability and convergence of finite element approximations of biot's consolidation problem. International Journal for Numerical Methods in Engineering, 37(4):645-667, 1994.

[40] LI, W.; WEI, C.. Minimum time-step size in transient finite element analysis of coupled poromechanical problems. Computers and Geotechnics, 98:197-204, 062018. 
[41] DE BORST, R.. The zero-normal-stress condition in plane-stress and shell elastoplasticity. Communications in Applied Numerical Methods, 7(1):29-33, 1991.

[42] MÜlLER, A. L.; VARGAS, E. D. A. ; VAZ, L. E.. Análise numérica da estabilidade de poços de petróleo considerando a variabilidade espacial e acoplamento fluido-mecânico. Phd thesis, Civil Engineering Department, Pontifícia Universidade Católica do Rio de Janeiro, Rio de Janeiro, 2007.

[43] STARMAN, B.; HALILOVIČ, M.; VRH, M. ; ŠTOK, B.. Consistent tangent operator for cutting-plane algorithm of elasto-plasticity. Computer Methods in Applied Mechanics and Engineering, 272:214 - 232, 2014.

[44] SIMO, J.; ORTIZ, M.. A unified approach to finite deformation elastoplastic analysis based on the use of hyperelastic constitutive equations. Computer Methods in Applied Mechanics and Engineering, 49(2):221 - 245, 1985.

[45] HUANG, J.; GRIFFITHS, D.. Observations on return mapping algorithms for piecewise linear yield criteria. International Journal of Geomechanics, 8, 072008.

[46] ORTIZ, M.; SIMO, J. C.. An analysis of a new class of integration algorithms for elastoplastic constitutive relations. International Journal for Numerical Methods in Engineering, 23(3):353-366, 1986.

[47] SIMO, J.; TAYLOR, R.. Consistent tangent operators for rateindependent elastoplasticity. Computer Methods in Applied Mechanics and Engineering, 48(1):101 - 118, 1985.

[48] BORJA, R.. Cam-clay plasticity, part ii: implicit integration of constitutive equation based a nonlinear elastic stress predictor. Applied Mechanics and Engineering, 88:225-240, 1991.

[49] BORJA, R.; TAMAGNINI, C.. Cam-clay plasticity part iii: Extension of the infinitesimal model to include finite strains. Computer Methods in Applied Mechanics and Engineering, 155:73-95, 1998.

[50] TURSKA, E.; SCHREFLER, B.. On convergence conditions of partitioned solution procedures for consolidation problems. Computer Methods in Applied Mechanics and Engineering, 106(1):51 - 63, 1993. 
[51] KIM, J.; TCHELEPI, H. ; JUANES, R.. Stability, accuracy and efficiency of sequential methods for coupled flow and geomechanics. SPE Journal, 16, 012009.

[52] QUARTERONI, A.; SACCO, R. ; SALERI, F.. Numerical Mathematics. Texts in Applied Mathematics. Springer New York, New York, NY, 2007.

[53] ZIENKIEWICZ, O. C.; PAUL, D. K. ; CHAN, A. H. C.. Unconditionally stable staggered solution procedure for soil-pore fluid interaction problems. International Journal for Numerical Methods in Engineering, 26(5):1039-1055, 1988.

[54] GUIDRY, G.; SPEZIA, K. ; SALMON, G.. Collaborative real-time analysis to reduce non-productive time. In: SPE ANNUAL TECHNICAL CONFERENCE AND EXHIBITION, Dallas, TX, Sep 2018.

[55] MCLEAN, M.; ADDIS, M.. Wellbore Stability Analysis: A Review of Current Methods of Analysis and Their Field Application. In: SPE/IADC DRILLING CONFERENCE. Society of Petroleum Engineers, 4 1990.

[56] RoChA, L. A. S.; AZEVEdo, C. T. D.. Projetos de Poços de Petróleo, volume 3. Editora Interciência, 2019.

[57] MÜLlER, A. L.; VARGAS, E. D. A.; VAZ, L. E. ; GONÇALVES, C. J.. Threedimensional analysis of boreholes considering spatial variability of properties and poroelastoplasticity. Journal of Petroleum Science and Engineering, 68(3-4):268-276, 2009.

[58] MÜLLER, A. L.; DO AMARAL VARGAS, E.; VAZ, L. E. ; GONÇALVES, C. J.. Borehole stability analysis considering spatial variability and poroelastoplasticity. International Journal of Rock Mechanics and Mining Sciences, 46(1):90-96, 2009.

[59] MA, T.; TANG, T.; CHEN, P. ; YANG, C.. Uncertainty evaluation of safe mud weight window utilizing the reliability assessment method. Energies, 12(5), 2019.

[60] MÜlleR, A. L.; VAZ, L. E.; VARGAS, E. D. A. ; GOnÇAlVES, C. J.. Procedimento numérico para determinação dos limites de pressão em problemas de instabilidade de poços de petróleo. Petro \& Química, p. 58-62, 062009. 
[61] MCDOUGALL, T. J.; WOTHERSPOON, S. J. ; BARKER, P. M.. An accelerated version of newton's method with convergence order $\sqrt{3}+1$. Results in Applied Mathematics, 4, 2019.

[62] ISAACSON, E.; KELLER, H.. Analysis of Numerical Methods. Dover Books on Mathematics. Dover Publications, 1994.

[63] FORD, J. A.. Improved illinois-type methods for the solution of the nonlinear equations. Scientia Iranica, 4(1):28-34, 1997.

[64] DOWELL, M.; JARRATT, P.. The "pegasus" method for computing the root of an equation. BIT Numerical Mathematics, 12(4):503-508, Dec 1972.

[65] IBAÑEZ, J. P.; MÜLLER, A. L. ; SANTI, M.. Hyper extended rifted margins: a computational procedure for stretching-thinning factors estimation. In: ANAIS DO XLIX CONGRESSO BRASILEIRO DE GEOLOGIA, p. 4951, Rio de Janeiro, 2018. XLIX Congresso Brasileiro de Geologia.

[66] SANTI, M.; MÜLLER, A. ; IBAÑEZ, J.. Kinematic and dynamic modeling for rift formation in crustal extensional mechanisms. In: CONFERENCE PROCEEDINGS, 79TH EAGE CONFERENCE AND EXHIBITION, volume 2017, p. 1-5. European Association of Geoscientists \& Engineers, 2017.

[67] RABElO, J.; BEZERRA, F. H. R.; SILVA, A. T. ; MORAES, A.. Efeito de tensões sobre o carste: implicações para reservatórios carbonáticos. In: ANAIS DO XLIX CONGRESSO BRASILEIRO DE GEOLOGIA, p. 7397, Rio de Janeiro, 2018. XLIX Congresso Brasileiro de Geologia.

[68] RABElO, J. G.; SILVA, A. T.; BEZERRA, F. H. R. ; MORAES, A.. Modelagem numérica da deformação em carbonatos e implicações na formação de carste. Geologia USP. Série Científica, 15(2):99-110, 62015.

[69] CONCEIÇÃO, T. F. L.; VARGAS, E. D. A.. Avaliação geomecânica de zonas de instabilidade, em poços de petróleo, abaixo de rochas evaporíticas. Master thesis, Departamento de Engenharia Civil, Pontifícia Universidade Católica do Rio de Janeiro, Rio de Janeiro, 2018.

[70] FETTER, M.; MORAES, A. ; MÜLLER, A. L.. Active low-angle normal faults in the deep water santos basin, offshore brazil: A geomechanical analogy between salt tectonics and crustal deformation. Geological Society, London, Special Publications, 458:SP458.11, 052017. 
[71] BRÊDA, T. C.; MORAES, A. ; MELLO, C. L.. Modelagem numérica da variação de campo neotectônico de paleotensões na porção emersa das bacias de campos e do espírito santo e áreas de embasamento adjacentes. In: ANAIS DO XLIX CONGRESSO BRASILEIRO DE GEOLOGIA, p. 8327, Rio de Janeiro, 2018. XLIX Congresso Brasileiro de Geologia.

[72] RODRIGUES, J. R. D. S. S.. Modelagem 2d aplicada ao estudo do desenvolvimento de zonas de falhas em depósitos da formação resende (bacia de volta redonda, rj). Master thesis, Instituto de Geociências, Universidade Federal do Rio de Janeiro, Rio de Janeiro, 2016.

[73] RABELO, J. G.. Criação de zonas de alta permeabilidade em fraturas: modelagem numérica do lajedo de soledade, formação jandaíra, bacia potiguar. Master thesis, Centro de Ciências Exatas e da Terra, Universidade Federal do Rio Grande do Norte, Natal, 2014.

[74] CELES, W.; PAULINO, G. ; ESPINHA, R.. A compact adjacency-based topological data structure for finite element mesh representation. International Journal for Numerical Methods in Engineering, 64:1529 - 1556, 112005.

[75] DUARTE, L. S.; CELES, W.. TopSim: A plugin-based framework for large-scale numerical analysis. Phd thesis, Informatics Department, Pontifícia Universidade Católica do Rio de Janeiro, Rio de Janeiro, 2016.

[76] HILL, R.. The Mathematical Theory of Plasticity. Oxford University Press, London, 1950.

[77] ROCSCIENCE. Stress analysis verification manual. Technical Report 164p., Rocscience, 2012.

[78] SALENCON, J. C. R.. Contraction quasi- statique d'une cavite a symetrie spherique ou cylindrique dans un milieu elastoplastique. Annales des Ponts et Chaussees, 139(4):231-236, July 1969.

[79] Detournay, E.; CHENG, A.. Poroelastic response of a borehole in a non-hydrostatic stress field. International Journal of Rock Mechanics and Mining Sciences \& Geomechanics Abstracts, 25(3):171-182, 1988.

[80] FREDRICH, J.; COBLENTZ, D.; FOSSUM, A. ; THORNE, B.. Stress perturbations adjacent to salt bodies in the deepwater gulf of mexico. In: PROCEEDINGS - SPE ANNUAL TECHNICAL CONFERENCE AND EXHIBITION, 102003. 
[81] GELET, R.; LORET, B. ; KHALILI, N.. Borehole stability analysis in a thermoporoelastic dual-porosity medium. International Journal of Rock Mechanics and Mining Science \& Geomechanics Abstracts, 50:65-76, Feb 2012.

[82] VASSILELLIS, K.; PARK, N.; PRASAD, U.; SAKOWSKI, S. A.; GRAHAM, B.; GHADIMIPOUR, A.; OLETU, J. ; LI, Y. L.. Gulf of mexico wilcox play property trend review. In: OFFSHORE TECHNOLOGY CONFERENCE, p. 9, Houston, Texas, USA, May 2013.

[83] POIATE JR, E.; MAIA, A. ; FALCAO, J. L.. Well Design for Drilling Through Thick Evaporite Layers. In: SPE/IADC DRILLING CONFERENCE AND EXHIBITION, 02 2006. SPE-99161-MS.

[84] COSTA, A. M.; POIATE, E.; AMARAL, C. S.; PEREIRA, A.; MARTHA, L. F.; GATTASS, M. ; ROEHL, D.. Geomechanics applied to the well design through salt layers in brazil: A history of success. In: Borja, R. I., editor, MULTISCALE AND MULTIPHYSICS PROCESSES IN GEOMECHANICS, p. 165-168, Berlin, Heidelberg, 2011. Springer Berlin Heidelberg.

[85] MAiA C., A.; POIATE J., E.; FAlCÃO, J. ; COElHO, L.. Triaxial Creep Tests in Salt Applied in Drilling Through Thick Salt Layers in Campos Basin-Brazil. In: SPE/IADC DRILLING CONFERENCE AND EXHIBITION, 02 2005. SPE-92629-MS.

[86] POIATE JR, E.; ROEHL, D. D. M. ; COSTA, L. M. D.. Mecânica das rochas e mecânica computacional para projeto de poços de petróleo em zonas de sal. Phd thesis, Departamento de Engenharia Civil, Pontifícia Universidade Católica do Rio de Janeiro, Rio de Janeiro, 2012.

[87] DE BORST, R.; CRISFIELD, M.; REMMERS, J. ; VERHOOSEL, C.. Nonlinear Finite Element Analysis of Solids and Structures. Wiley Series in Computational Mechanics. Wiley, 2012.

[88] ZIENKIEWICZ, O.; TAYLOR, R. ; NITHIARASU, P.. The Finite Element Method for Fluid Dynamics. Elsevier Science, 2005.

[89] CORREIA, U. M.; BATEZELLI, A. ; LEITE, E. P.. 3-D Geological modelling: a siliciclastic reservoir case study from Campos Basin, Brazil. REM - International Engineering Journal, 69:409 - 416, 122016. 